\title{
Phenotypic and molecular evolution across 10,000 generations in laboratory budding yeast populations
}

\begin{abstract}
Milo S. Johnson ${ }^{1,2,3}$, Shreyas Gopalakrishnan ${ }^{1,2,3,4}$, Juhee Goyal ${ }^{1,8}$, Megan E. Dillingham ${ }^{2,5}$, Christopher W. Bakerlee ${ }^{1,2,3,4}$, Parris T. Humphrey ${ }^{1,2,3}$, Tanush Jagdish ${ }^{1,2,3,5}$, Elizabeth R. Jerison $^{1,6,12}$, Katya Kosheleva ${ }^{1,6}$, Katherine R. Lawrence ${ }^{1,2,3,7}$, Jiseon Min $^{1,2,3,4,8}$, Alief Moulana $^{1}$, Angela M. Phillips ${ }^{1}$, Julia C. Piper ${ }^{1,11}$, Ramya Purkanti ${ }^{1,10}$, Artur Rego-Costa ${ }^{1}$, Michael J. McDonald ${ }^{1,13}$, Alex N. Nguyen $\mathrm{Ba}^{1,2,3,9,14}$, and Michael M. Desai ${ }^{1,2,3,6}$

${ }^{1}$ Department of Organismic and Evolutionary Biology, Harvard University, Cambridge, MA 02138, USA; ${ }^{2}$ Quantitative Biology Initiative, Harvard University, Cambridge, MA 02138, USA; ${ }^{3}$ NSF-Simons Center for Mathematical and Statistical Analysis of Biology, Harvard University, Cambridge, MA 02138, USA; ${ }^{4}$ Department of Molecular and Cellular Biology, Harvard University, Cambridge, MA, USA; ${ }^{5}$ Graduate Program in Systems, Synthetic, and Quantitative Biology, Harvard University, Cambridge, MA, USA; ${ }^{6}$ Department of Physics, Harvard University, Cambridge, MA, USA; ${ }^{7}$ Department of Physics, Massachusetts Institute of Technology, Cambridge, MA, USA; ${ }^{8} \mathrm{John}$ A. Paulson School of Engineering and Applied Sciences, Harvard University, Cambridge, MA, USA; ${ }^{9}$ Department of Cell and Systems Biology, University of Toronto, Toronto, Canada; ${ }^{10}$ The Max Planck Institute of Molecular Cell Biology and Genetics, Dresden, Germany; ${ }^{11}$ AeroLabs, Aeronaut Brewing Co, Somerville, MA, USA; ${ }^{12}$ Department of Applied Physics, Stanford University, United States; ${ }^{13}$ School of Biological Sciences, Monash University, Victoria, Monash, Australia; ${ }^{14}$ Department of Cell and Systems Biology, University of Toronto, Toronto, Canada
\end{abstract}

\section{Abstract}

Laboratory experimental evolution provides a window into the details of the evolutionary process. To investigate the consequences of long-term adaptation, we evolved $205 \mathrm{~S}$. cerevisiae populations (124 haploid and 81 diploid) for 10,000 generations in three environments. We measured the dynamics of fitness changes over time, finding repeatable patterns of declining adaptability. Sequencing revealed that this phenotypic adaptation is coupled with a steady accumulation of mutations, widespread genetic parallelism, and historical contingency. In contrast to long-term evolution in E. coli, we do not observe long-term coexistence or populations with highly elevated mutation rates. We find that evolution in diploid populations involves both fixation of heterozygous mutations and frequent loss-of-heterozygosity events. Together, these results help distinguish aspects of evolutionary dynamics that are likely to be general features of adaptation across many systems from those that are specific to individual organisms and environmental conditions. 


\section{Introduction}

As human health is increasingly threatened by emerging pathogens, multidrug resistant infections, and therapy-evading cancer cells, our understanding of the dynamics and predictability of evolution is of growing importance. Yet predicting the course of evolution is difficult, since it is driven by a complex combination of deterministic and stochastic forces. On the one hand, beneficial mutations that establish within a population often rise to fixation at rates nearly perfectly predicted by decades-old theory. On the other hand, random forces such as mutation, genetic drift, and recombination ensure an enduring role for chance and contingency. To understand evolution, we must appreciate the interactions between these deterministic and stochastic components.

While there is extensive theoretical work analyzing how the interplay between these factors affects the rate, predictability, and molecular basis of evolution, empirical evidence remains relatively limited. In large part this stems from a basic difficulty: we cannot easily characterize the predictability of evolution using observational studies of natural populations, because we cannot replicate evolutionary history. In addition, the inferences we can make from extant populations and the fossil record are limited by a lack of complete data.

To circumvent these difficulties, scientists have turned to laboratory evolution experiments, primarily in microbial populations. These provide a simple model system in which researchers can maintain many replicate populations for hundreds or thousands of generations, in a setting where the environment and other relevant parameters (e.g. population size) can be precisely controlled and manipulated. By conducting phenotypic and sequencing studies of the resulting evolved lines, we can observe evolution in action, and ask whether specific phenotypic and genotypic outcomes are predictable.

Over the last several decades, a few consistent results have emerged from these types of experiments (reviewed in Kassen (2014)). As populations evolve in a constant environment, they gain fitness along a fairly predictable trajectory, following a pattern of declining adaptability in which the rate of fitness increase slows as populations adapt (Couce and Tenaillon, 2015; Kryazhimskiy et al., 2014; Wiser et al., 2013). Meanwhile, the rate of molecular evolution remains roughly constant (Barrick et al., 2009; Good et al., 2017; Tenaillon et al., 2016). Mutations are rarely predictable at the nucleotide level but often moderately predictable at higher levels: mutations in certain genes or pathways are repeatedly fixed across replicate populations (Bailey et al., 2015; Kryazhimskiy et al., 2014; Tenaillon et al., 2012, 2016). Phenotypes not under direct selection change less predictably than fitness in the evolution environment, but sometimes still exhibit some correlation with level of adaptation in the evolution environment (Jerison et al., 2020; Leiby and Marx, 2014; Ostrowski et al., 2005).

Most of these microbial and viral evolution experiments, as well as those in multicellular eukaryotes such as $C$. elegans and Drosophila melanogaster, involve at most about 1,000 
generations of adaptation to a novel environment. This makes them well suited to studying the initial dynamics of adaptation, where a population encounters a novel environment and rapidly acquires beneficial mutations as it evolves in response to this new challenge. However, it is unclear how far we can extrapolate findings from this type of study. Will evolutionary dynamics remain similar over longer timescales? Or will the evolutionary dynamics change in qualitative ways once a population has had thousands of generations to become well-adapted to the laboratory environment?

The experiment best equipped to answer this question is the Long-Term Evolution Experiment (LTEE) conducted by Richard Lenski and collaborators. For over 30 years and 70,000 generations (reviewed in Lenski (2017)), the Lenski lab has propagated 12 Escherichia coli populations in minimal media by batch culture. The LTEE has led to numerous insights into evolutionary dynamics over both short and long timescales, and has also provided many examples of interesting phenomena such as contingency (Blount et al., 2012; Good et al., 2017), the spontaneous emergence of quasi-stable coexistence (Good et al., 2017; Plucain et al., 2014; Rozen and Lenski, 2000), and evolution of mutation rates (Sniegowski et al., 1997; Wielgoss et al., 2013). The LTEE is unique among microbial evolution experiments in its long timescale, and provides an important look at evolution well beyond the initial rapid adaptation of a population to a novel laboratory environment. However, it is limited by its specificity: it involves twelve replicate populations, each founded from a single $E$. coli strain, all evolving in the same constant environment. It thus remains unclear which of the broad conclusions drawn from this experiment will be generalizable to other organisms and environments. Would we draw similar conclusions when other species are allowed to evolve in other environments for long periods of time?

While no other laboratory evolution experiments match the LTEE in timescale, a few have extended beyond the $\sim 1,000$ generations of most other experiments. For example, Behringer et al. (2018) evolved E. coli populations in tubes for up to 10,000 generations and found that they repeatedly evolved a biofilm phenotype and stable coexisting subpopulations. Fisher et al. (2018) evolved laboratory populations of the budding yeast $S$. cerevisiae for 4,000 generations, finding that as in $E$. coli, these populations gain fitness along predictable trajectories characterized by declining adaptability. This experiment, along with Marad et al. (2018), also studied the relationship between ploidy and adaptation, finding that in general diploids adapt more slowly than haploids, presumably due to the inaccessibility of recessive beneficial mutations (Zeyl 2003; Marad et al. 2018; Fisher et al. 2018). While these experiments provide an important first look into long-term adaptation in yeast and $E$. coli, they all involve relatively limited whole-population sequencing, and none have provided data on the dynamics of molecular evolution in both haploid and diploid populations over many thousands of generations. 


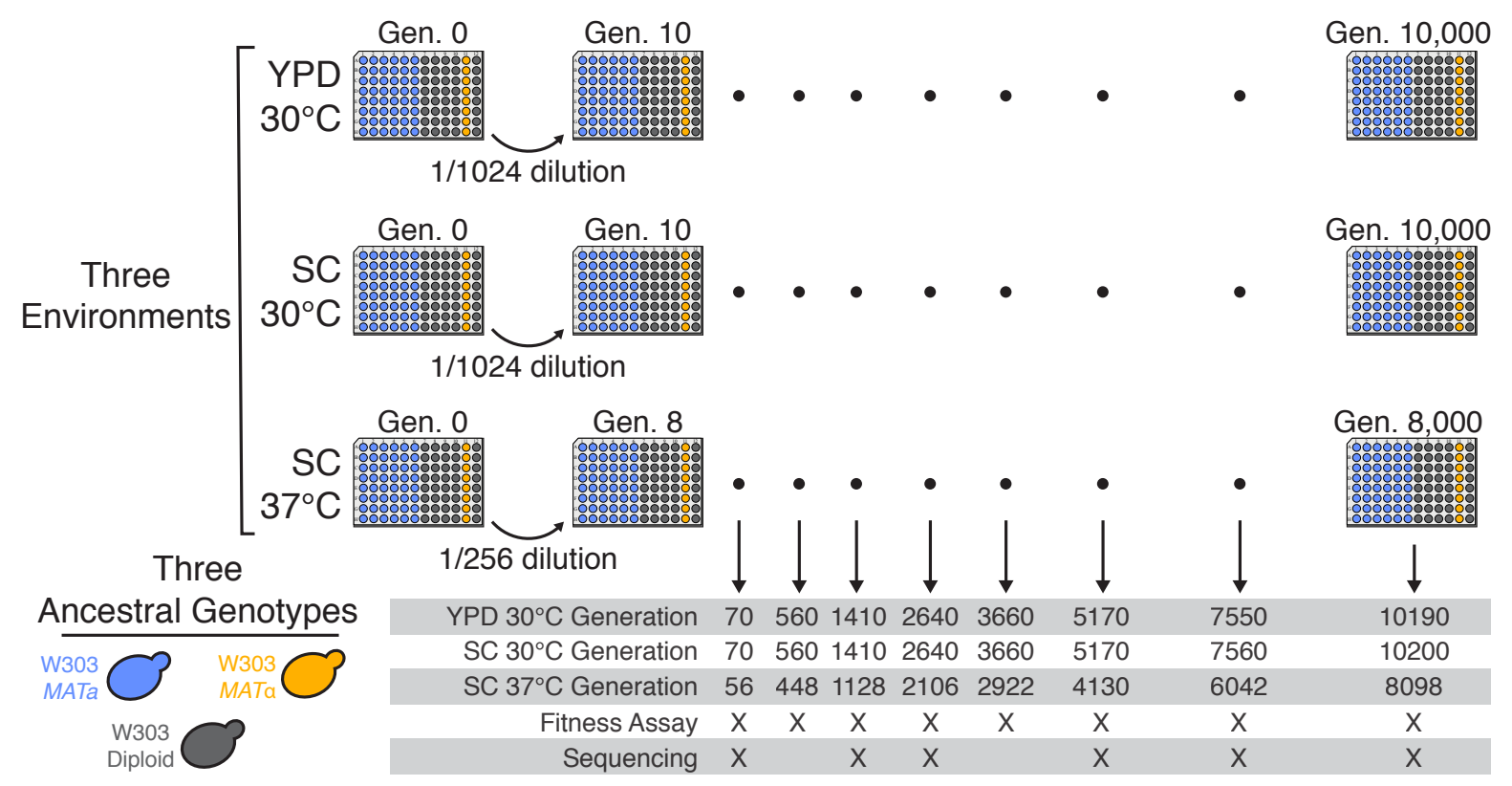

Figure 1. Experimental design. We propagated budding yeast lines in 96-well microplates in one of three environmental conditions, using a daily dilution protocol as shown at top. Each population was founded by a single clone of one of three ancestral genotypes (a haploid MATa, a haploid $M A T \alpha$, and a diploid, all derived from the W303 strain background). On a weekly basis, we froze all populations in glycerol at $-80^{\circ} \mathrm{C}$ for long-term storage. The frozen timepoints used for the analyses in this paper are indicated at bottom.

To fill this gap, we established a long-term evolution experiment in the spirit of the LTEE, with a total of 205 budding yeast populations (split between haploids and diploids) evolving in three different laboratory environments. In this paper, we describe the first 10,000 generations of this experiment. We find that some aspects of evolution in our system are broadly consistent with the conclusions of the LTEE and other long-term evolution experiments. For example, the dynamics of fitness increase are largely repeatable between replicate lines and show a pattern of declining adaptability over time even while the rate of molecular evolution remains relatively constant. However, there are also key differences: we find no evidence of stably coexisting lineages or widespread evolution of mutator phenotypes. As the first laboratory evolution of this length in a eukaryotic system, our study provides an important test of the generality of conclusions from earlier work (primarily the LTEE), as well as a novel opportunity to observe evolutionary dynamics over long timescales across many replicate populations in multiple environmental conditions. 


\section{Results}

We founded 45 haploid mating type a (MATa), 8 mating type $\alpha(M A T \alpha)$, and 37 diploid $S$. cerevisiae populations in each of three evolution environments (90 populations per environment, for a total of 270 independent lines; see Figure 1). Each population was founded from a single independent colony of the corresponding ancestral W303 MATa, $M A T \alpha$, or diploid strain (see Methods for details). We then propagated each population in batch culture in one well of an unshaken 96-well microplate in the appropriate environment (YPD at $30^{\circ} \mathrm{C}$, SC at $30^{\circ} \mathrm{C}$, and SC at $37^{\circ} \mathrm{C}$ ), with daily $1: 2^{10}$ dilutions for the $30^{\circ} \mathrm{C}$ environments, and $1: 2^{8}$ dilutions for the $37^{\circ} \mathrm{C}$ environment. We froze glycerol stocks of each population every week (corresponding to every 70 generations in the $30^{\circ} \mathrm{C}$ environments, and every 56 generations in the $37^{\circ} \mathrm{C}$ environment), creating a frozen fossil record for future analysis. A total of 65 populations were lost during the first 10,000 generations of evolution due to contamination, evaporation, or pipetting errors (see Methods for details; Figure 1 - figure supplement 1), leaving us with 205 populations.

\section{Fitness changes during evolution}

To measure changes in fitness over time, we unfroze populations from 8 timepoints in each of the 205 evolved populations (see Figure 1) and conducted competitive fitness assays against a fluorescently labeled reference strain (see Methods for details). In Figure 2 , we show the resulting fitness trajectories in each population. We find that in most cases, including almost all haploid populations, these trajectories tell a familiar story of declining adaptability: populations predictably increase in fitness rapidly in the first few hundred generations, and then adapt more slowly as time progresses (Figure 2 - figure supplement 1A). We find a different pattern in some diploid populations, where an initial slower period of fitness gain is succeeded by a significant rapid increase in fitness. However, even in these cases, populations at lower fitness midway through the experiment tend to adapt more quickly and "catch up" in the second half of the experiment (Figure 2). We also find that a few populations (indicated by asterisks in Figure 2) experience dramatic increases in fitness, and subsequently remain at higher fitness than other populations for the duration of the experiment. As we describe in more detail below, these events are caused by a specific mutation in the adenine biosynthesis pathway (see "ADE pathway mutations" section).

On average, our haploid populations gained more fitness over the course of evolution than diploids ( $P<0.02$, Mann-Whitney U test), consistent with prior work (Fisher et al., 2018; Marad et al., 2018; Zeyl, 2003). This effect could be entirely due to reduced accessibility of recessive beneficial mutations in diploids (as these previous studies propose). However, we note that this effect is also consistent with declining adaptability: the diploids have a higher ancestral fitness than the haploids in all three environments (Figure 2). In agreement with this hypothesis, we find that in $\mathrm{SC} 30^{\circ} \mathrm{C}$, diploids adapt much slower than haploids during the first half of the evolution, but gain significantly more fitness than either haploid strain background in the second half of evolution (Figure 2 - figure 

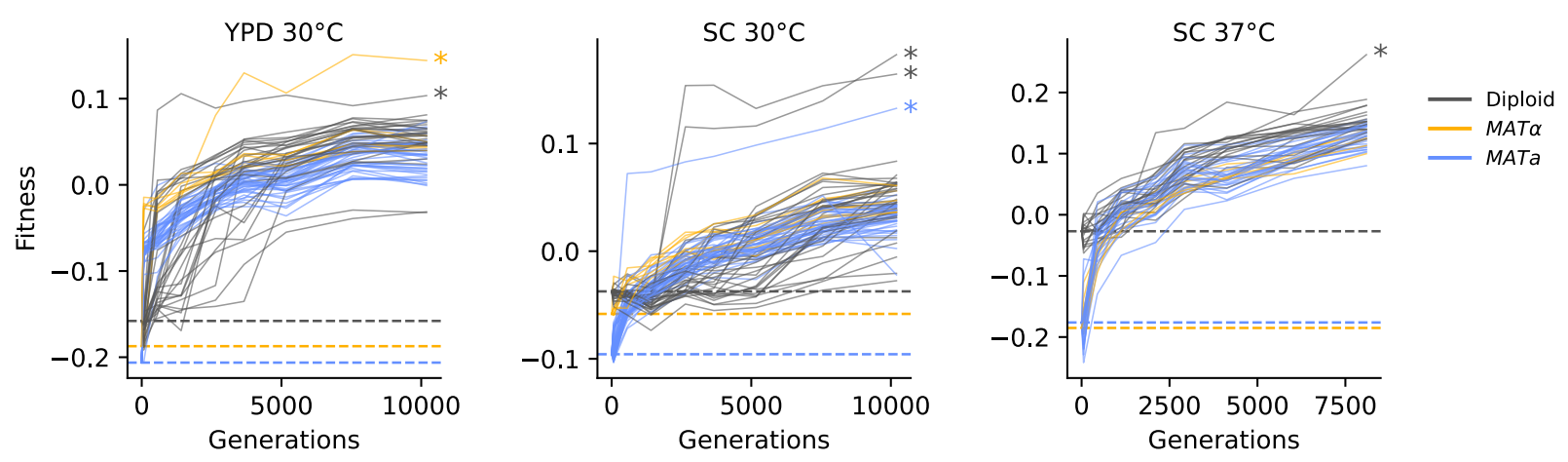

Figure 2. Fitness changes during evolution. Competitive fitness is plotted relative to a reference strain in each environment. Inferred ancestral fitness is indicated by horizontal lines and colored by strain. Populations with premature stop-codon reversion mutations in ADE2 are indicated by asterisks. Correlations between replicate fitness measurements are shown in Figure 2 - figure supplement 2 .

supplement 1B; $P<0.02$, Mann-Whitney $\mathrm{U}$ test). This is also consistent with the more rapid evolution of MATa haploids compared to MAT $\alpha$ haploids in SC $30^{\circ} \mathrm{C}$, where the MATa haploids start at lower fitness (Figure 2 - figure supplement 1B; $P<0.02$, Mann-Whitney U test). Together, these results are consistent with a picture in which the pattern of declining adaptability as a function of fitness applies not only along the course of a fitness trajectory for one population, but also between ancestral strains of different ploidy and mating type. This effect tends to draw fitness trajectories together as evolution proceeds, leading almost all populations to a similar final fitness.

\section{Molecular evolution}

At six of the timepoints used for fitness assays (Figure 1), we also performed wholepopulation, whole-genome sequencing in 90 focal populations (12 MATa, 12 diploid, and 6 $M A T \alpha$ from each environment). After aligning sequencing reads and calling variants, we use observed allele counts across multiple timepoints to filter out sequencing and alignment errors and identify a set of mutations present in each evolving population (Methods). At each sequenced timepoint, we call mutations fixed if they are at greater than or equal to $40 \%$ frequency (diploids) or $90 \%$ frequency (haploids) and do not drop below these thresholds at a later timepoint. We additionally call loss of heterozygosity in mutations in diploids using the criteria for fixation in haploids ( $90 \%$ threshold).

Our data shows that mutations fix steadily through time across all sequenced populations (Figure 3). While we would need more sequenced timepoints to fully observe the frequency trajectories of mutations in these populations, we can see a few patterns from our temporally sparse sequencing (Figure 3A, Figure 3 figure supplements 1-9). We frequently observe clonal interference in which groups of mutations rise to high frequency and then plummet to extinction, outcompeted by another group. All populations fix mutations throughout the experiment; we find no evidence for the 
A

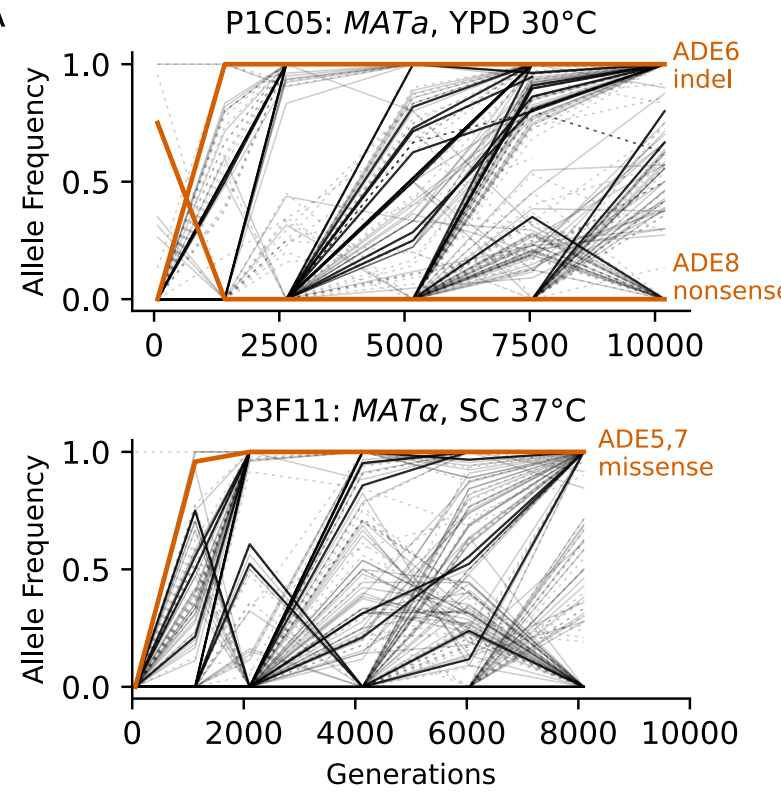

P2F07: Diploid, $\mathrm{SC} 30^{\circ} \mathrm{C}$

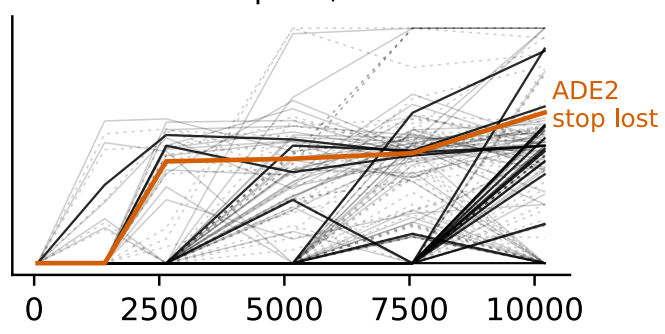

P1F10: Diploid, YPD $30^{\circ} \mathrm{C}$

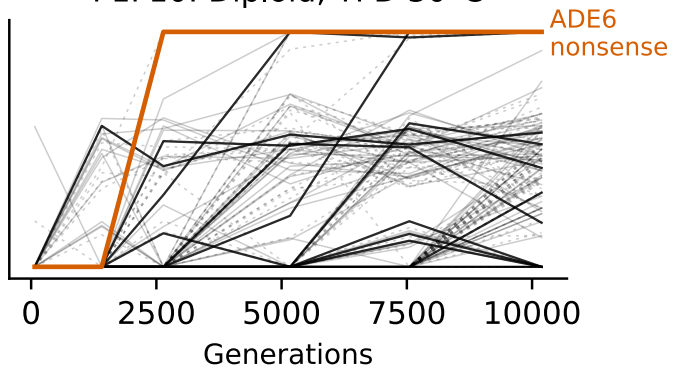

B
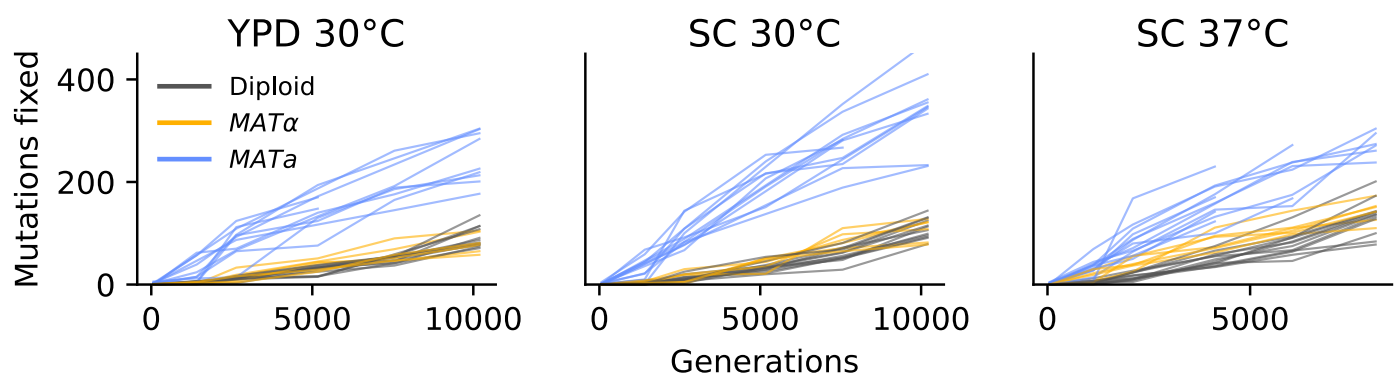

Figure 3. Dynamics of molecular evolution. (A) Allele frequencies over time in four example populations. Nonsynonymous mutations in "multi-hit" genes are solid black lines (see "Parallelism" section below), nonsynonymous mutations in the adenine biosynthesis pathway are colored orange and labeled, other nonsynonymous mutations are thin grey lines, and synonymous mutations are dotted lines. (B) Number of fixed mutations over time in each population. Timepoints with average coverage less than 10 (for haploids) or 20 (for diploids) are not plotted.

emergence of stably coexisting lineages within any of our populations (Figure 3B, Figure 3 - figure supplement 10). Denser sequencing through time would be required to determine whether any populations exhibit shorter periods of semistable coexistence (e.g. as seen by Frenkel et al. (2015)). It is also possible we are missing coexistence of haplotypes at very low frequency ( $\leqslant 5 \%$ ), which sequencing may not be able to detect. However, our results rule out long-term coexistence of multiple lineages at substantial frequencies like that observed in the LTEE or Behringer et al. (2018).

We find that the rate of mutation accumulation in the MATa populations is consistently higher than in MAT $\alpha$ or diploid populations (Figure 3B). This is likely due to a higher 
A

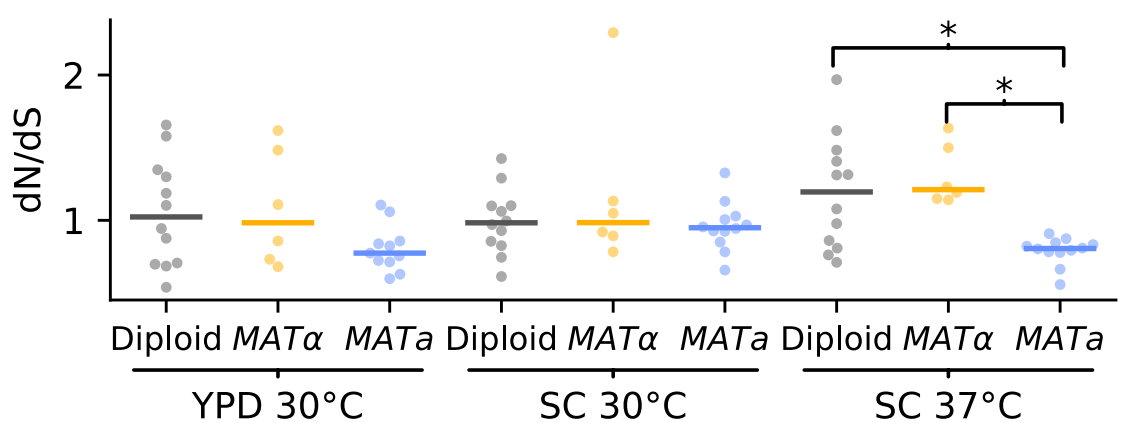

B

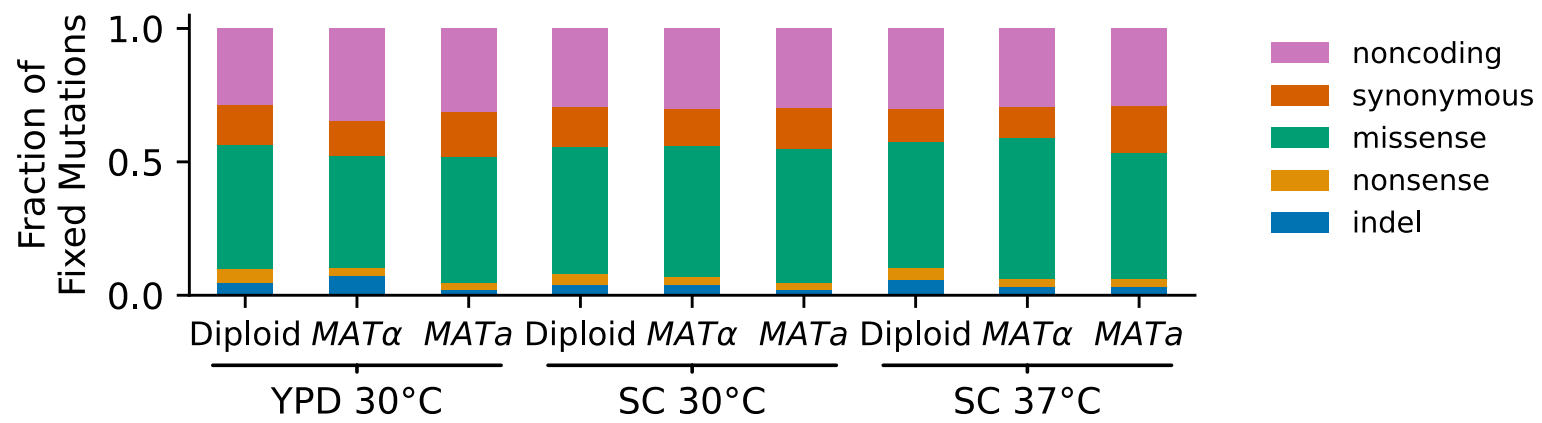

Figure 4. Types of mutations. (A) Swarm plot of dN/dS (ratio of nonsynonymous / synonymous fixations by the final timepoint, scaled by the ratio of possible nonsynonymous / synonymous mutations across the genome) for each environment-strain combination. Each point represents one population and the horizontal line represents the median. Asterisks indicate significant differences $(P<0.01$, Mann-Whitney U test) between strains in the same environment. (B) Breakdown of mutation types for all mutations fixed by the final timepoint, in all populations corresponding to each environment-strain combination.

mutation rate in our MATa ancestor. Consistent with this hypothesis, we find that MATa populations have a lower ratio of nonsynonymous to synonymous mutations than MAT $\alpha$ or diploid populations in all three environments, as expected if a higher mutation rate leads to an increase in hitchhiking (though we note that this comparison is only significant in SC $37^{\circ} \mathrm{C}$; $P<0.01$, Mann-Whitney U Test, figure $4 \mathrm{~A}$ ). We identified a putative causal mutation in TSA1 in our MATa ancestor; this mutation is absent in our MAT $\alpha$ ancestor and heterozygous in our diploid ancestor. We confirmed that the TSA1 mutation increases mutation rate in a BY strain background (Figure 4 - figure supplement 1).

Overall, we find that $\mathrm{dN} / \mathrm{dS}$ ratios for fixed mutations in our populations are near one (Figure 4A), suggesting that selection in favor of beneficial (and presumably typically nonsynonymous) mutations is balanced by hitchhiking of neutral mutations and purifying selection against deleterious mutations. The relative prevalence of different types of fixed mutations across strains and environments are similar, with roughly $45-50 \%$ missense mutations, $40-45 \%$ synonymous and noncoding mutations, and 5-10\% nonsense and indel 
mutations (Figure 4B). While there is variation between populations in the number of mutations accumulated, we do not observe any sudden increases in the rate of mutation accumulation (Figure 3B). This stands in contrast to the LTEE, where mutator alleles sweep to fixation and dramatically increase the mutation rate in 6 of 12 replicate populations. We do observe one potential mutator event: P1E11, a MAT $\alpha$ population evolved in YPD $30^{\circ} \mathrm{C}$ has an unusually large number of indel mutations, likely due to a mutation in the mismatch repair protein MSH3 that hitchhiked to fixation with an indel mutation in GPB2 (Figure 4 - figure supplement 2). However, the elevation in mutation rate in this population remains relatively modest. While we do observe mutations in mutator-associated genes such as MSH3 in other populations, we do not observe clear differences in mutation-type distribution or rate of mutation accumulation in these populations, suggesting that these mutations lead to at most subtle changes in mutation rate (stacked mutation type plots for each population are shown in Figure 4 - figure supplements 3-11). Further work will be needed to characterize more subtle variation in mutation rate in each of these populations.

\section{Parallelism}

Next, we examined whether mutations in certain genes are fixed more frequently than we would expect by chance. We define a "hit" as a nonsynonymous mutation that is fixed by the final timepoint, and define the multiplicity of a gene as the number of hits in that gene across all sequenced populations, divided by its relative target size (Good et al., 2017). As in many other laboratory evolution experiments, we observe an excess of high multiplicity genes in our data, relative to a null in which mutations are fixed randomly across all open reading frames (Figure 5A).

A

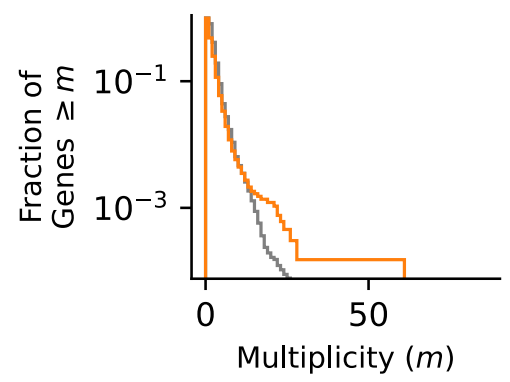

B

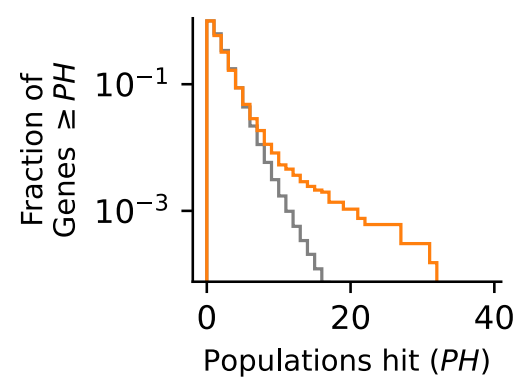

C

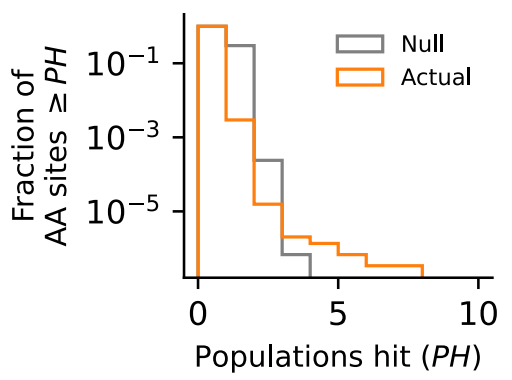

Figure 5. Parallelism. Comparison between null and actual distributions of (A) the fraction of genes with multiplicity $\geq m$ (see Methods), (B) the fraction of genes with hits in $\geq P H$ populations, and (C) the fraction of amino acid sites with hits in $\geq P H$ populations (those with $P H \geq 3$ are listed in Figure 5 - figure supplement 1). For all three plots, the null distribution (shown in gray) is obtained by simulating random hits to genes, taking into account the number of hits in each population in our data and the relative length of each gene. 


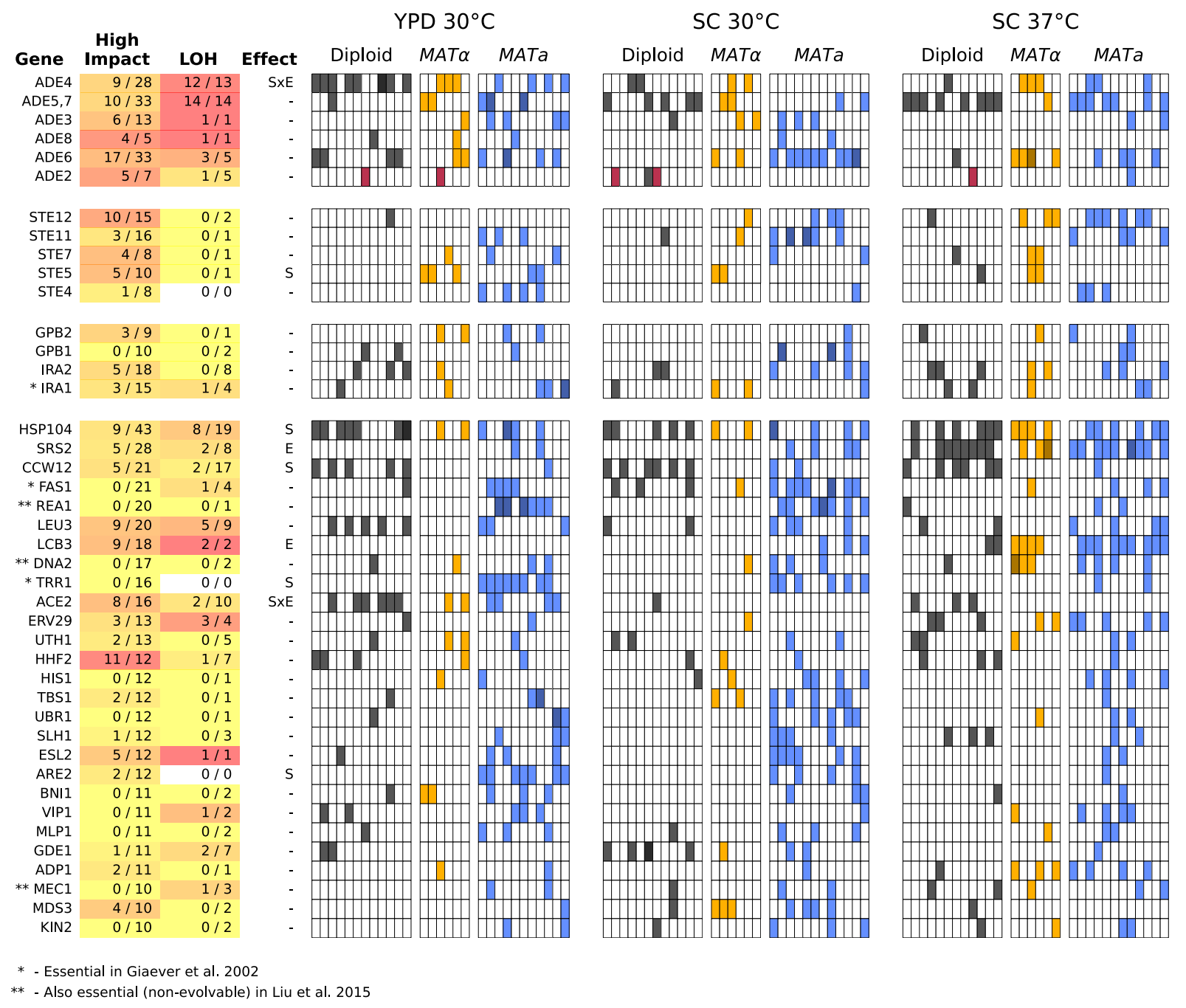

Figure 6. Multi-hit genes. Each row represents a gene. The first three blocks are groups of genes identified from gene-ontology enrichment analysis of multi-hit genes (from top to bottom: adenine biosynthesis, sterility, and negative regulation of the Ras pathway). The bottom block is all other genes with hits in at least 10 populations. Each column in the heatmap represents a population, such that if a gene is hit in that population the square will be colored (darker color if a gene is hit 2 or more times in that population). Red squares indicate premature-stop-lost mutations in ADE2, which correspond to the populations with asterisks in Figure 2. One population that was not sequenced (not shown here) also has this mutation (confirmed by Sanger sequencing). The table at left gives more information on each multi-hit gene: "High impact” is the fraction of hits that are likely to cause a loss-of-function, as annotated by SnpEff (e.g. nonsense mutations), "LOH" (loss of heterozygosity) is the fraction of hits in diploid populations that fix homozygously, and "Effect" describes whether the hits are distributed significantly unevenly across strain-types (S), environments (E), or both (SxE), when compared to a null model where fixations are not strain or environment dependent. 
To understand the functional basis of this parallelism, we focus on multi-hit genes, defined as those with hits in six or more populations. These multi-hit genes (Figure 6) are enriched for several gene ontology (GO) terms (Figure 6 - figure supplement 1), indicating parallelism at the level of biosynthetic and signaling pathways. In Figure 6 we show all genes with hits in ten or more populations, and highlight several key functional groups (adenine biosynthesis, sterility, and negative regulators of the Ras pathway; see Figure 6 figure supplements 2-4 for analogous figures for all other multi-hit genes). Mutations in the latter two functional groups are commonly observed in yeast evolution experiments, and have been shown to be beneficial in similar environments (Echenique et al., 2019; Kryazhimskiy et al., 2014; Lang et al., 2013; Venkataram et al., 2016). The mutations in adenine biosynthesis, by contrast, reflect the particular genotype of our ancestral strains; we discuss these further below.

We next asked whether some multi-hit genes are more likely to fix mutations in particular strain backgrounds or environments. We find that most multi-hit genes have mutations distributed across both haploid mating types, diploids, and all three environmental conditions, indicating that these mutations are presumably beneficial in all of these contexts. However, we do find several mutations that are either strain or environment specific ("Effect" column in Figure 6; Figure 6 - figure supplement 5, Supplemental Data). For example, mutations in SRS2 and LCB3 are fixed more often in SC $37^{\circ} \mathrm{C}$, while mutations in CCW12 are fixed more in diploids.

To investigate the impact of the mutations in multi-hit genes on protein function, we used SnpEff (Cingolani et al., 2012) to predict the impact of each mutation. In Figure 6, we show the fraction of mutations in each multi-hit gene that were annotated as "High Impact." Because most of these high-impact mutations are nonsense or frameshift mutations, they are very likely to lead to loss of function of the associated gene, as are some fraction of the "Moderate Impact” mutations (e.g. some missense mutations or in-frame deletions). We find that many of our multi-hit genes have a large percentage of high impact mutations, suggesting that selection acts in favor of loss-of-function of the corresponding genes, consistent with many earlier laboratory evolution experiments (Murray, 2020). However, this is not universal: a few genes with 10 or more hits have no high-impact mutations fixed, and several of these genes are essential (Figure 6). This suggests that selection in these genes may be instead for change- or gain-of-function.

\section{ADE pathway mutations}

The founding genotype of all the populations used in this experiment is derived from the W303 strain background, which has a premature stop codon in the ADE2 gene (ade2-1). This disrupts the adenine biosynthetic pathway, which is likely deleterious because adenine depletion can limit growth even in rich media (Kokina et al., 2014). In addition, loss-of-function of ADE2 causes buildup of a toxic intermediate, phosphoribosylaminoimidazole (AIR), which is converted to a visible red pigment that accumulates 
A

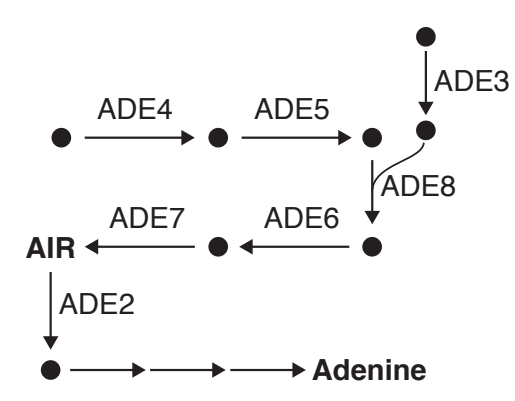

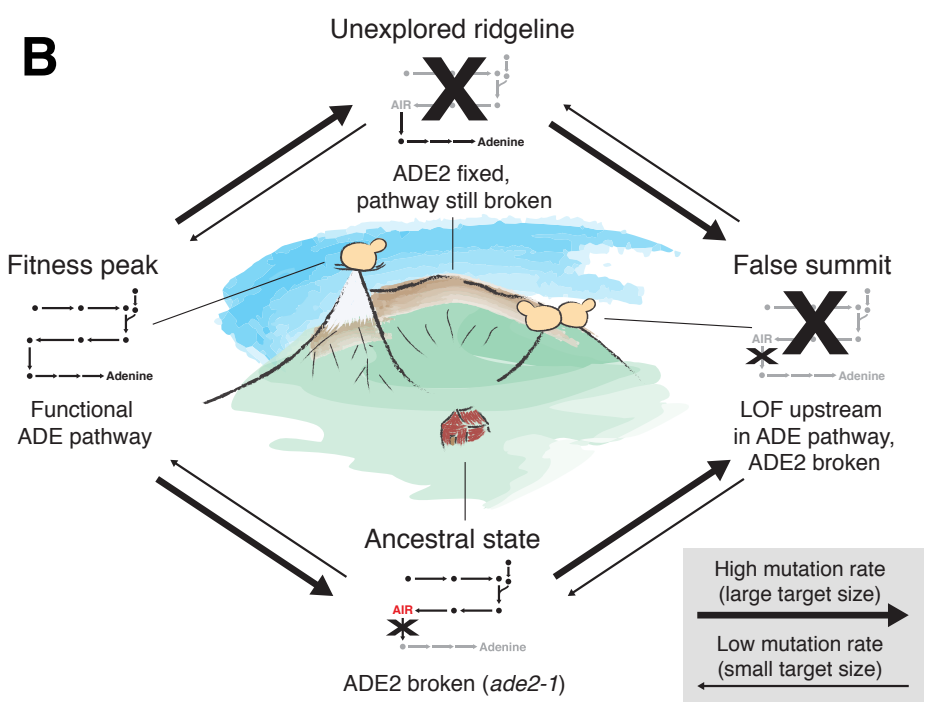

Figure 7. ADE pathway evolution. (A) Simplified schematic of the adenine biosynthesis pathway. Circles represent metabolic intermediates; AIR is the toxic metabolic intermediate phosphoribosylaminoimidazole. (B) Schematic of a fitness landscape with four possible states defined by whether ADE2 is functional and whether the ADE pathway upstream of ADE2 is functional. The small insets represent the state of the pathway in (A) at each position. Elevation in the landscape represents putative fitness differences, and the width of the arrows represents the putative mutation rates between the different states.

in the vacuole (Kokina et al., 2014; Sharma et al., 2003) (Figure 7A). This means that lossof-function mutations upstream in this pathway, which are deleterious when ADE2 is functional because they disrupt adenine biosynthesis, are strongly beneficial in the ade21 background because they prevent this toxic buildup (Echenique et al., 2019). Consistent with this, we see rapid fixation of at least one mutation in the ADE pathway, typically upstream of ADE2, in almost all of our sequenced populations, along with frequent loss of heterozygosity of these mutations in diploids (Figure 3A).

Five of our sequenced populations find a better solution: they fix mutations that revert the premature stop codon so that the full ADE2 sequence can be translated (populations indicated by asterisks in Figure 2 and mutations shown in red in Figure 6; note that one unsequenced high-fitness population also has this mutation, confirmed by Sanger sequencing). These populations have higher fitness than other populations from the same strain background and environment, presumably because they have both repaired the defect in adenine biosynthesis and avoided the buildup of the toxic intermediate. As we would expect, these populations do not fix any loss-of-function mutations in other ADE pathway genes. The fact that only six of our populations find this higher-fitness reversion of ade2-1 is presumably a consequence of differences in target size: while loss-of-function 
in genes upstream in the pathway can arise from a variety of mutations in five genes upstream of ADE2, the ade2-1 reversion requires a mutation at a specific codon in ADE2.

We note that once a population has fixed an upstream loss-of-function mutation, it requires reversion of both the original ade2-1 mutation and the upstream mutation to find the higher-fitness genotype. While this is possible in principle, both mutations have singlecodon target sizes and when they occur alone are likely neutral and deleterious respectively, making this evolutionary path extremely improbable. We do not observe any populations that move from the lower fitness genotype to the higher fitness genotype even after 10,000 generations of evolution. Figure 7 depicts these evolutionary states using a simple fitness landscape framework.

\section{Contingency}

The alternative evolutionary paths involving mutations in the ADE pathway are an example of contingency that is already well understood (Echenique et al., 2019; Roman, 1956). We next sought to analyze the role of contingency more broadly in our experiment. To do so, we first analyzed whether mutations are over-dispersed or under-dispersed among populations, following Good et al. (2017). Looking within each environment-strain combination, we find that mutations are more over-dispersed than expected by chance; this is still true if we also include mutations that are present but not fixed (Figure 7 - figure supplement 1). This provides evidence of “coupon collecting”: populations with a fixed nonsynonymous mutation in a gene are less likely to fix another mutation in that gene.

We next sought to test whether mutations in a given gene tend to open up or close off opportunities for beneficial mutations in other genes. To do so, we calculated the mutual information between multi-hit genes (i.e. for each pair of multi-hit genes, whether a population with a fixed nonsynonymous mutation in the first gene is more or less likely to have a fixed nonsynonymous mutation in the second). As in Fisher et al. (2019), we find that the sum of mutual information across all pairs of multi-hit genes in our experiment is higher than in simulations ( $P=0.03$, Figure 7 - figure supplement 2$)$. Thus there is an overall statistical signature of contingency in our data: mutations in certain genes make mutations in others more or less likely. However, we do not have power to isolate this signature to individual pairs of genes; the mutual information between any two multi-hit genes in our experiment is not higher than we would expect by chance. Note that because we calculate mutual information separately for each environment-strain combination (at most 12 populations per group), we have less power than Fisher et al. (2019) to detect interactions between genes. In sum, while we cannot confidently identify more specific examples of contingency in our data beyond a general pattern of coupon-collecting, it is likely to be playing a role, as in the LTEE (Good et al., 2017). 


\section{Patterns of molecular evolution specific to diploids}

Our experiment provides an opportunity to compare asexual adaptation in diploids to that in haploids, and to characterize diploid-specific aspects of the evolutionary dynamics (Figure 8). In contrast to Fisher et al. (2018), only one of our focal haploid populations underwent a whole genome duplication and became diploid during our experiment (Figure 8 - figure supplements 1-2, and see Methods). We have excluded this population from all comparative analyses, and we restrict our analysis of diploids here to the diploid populations started from a diploid ancestor.

A key difference between evolution in diploids and haploids has to do with the dominance effects of mutations. Some mutations that provide a fitness advantage in haploids may be fully or partially dominant in diploids, and hence provide a fitness advantage when they initially arise in a single chromosome. Others are likely to be recessive, and hence are neutral when they initially arise in a single chromosome. Earlier laboratory evolution experiments have found that diploid populations of budding yeast tend to adapt more slowly than haploids, which could be a signature of the impact of Haldane's sieve (e.g. if most beneficial mutations in haploids are loss-of-function mutations and most loss-offunction mutations are recessive) (Fisher et al., 2018; Marad et al., 2018; Zeyl, 2003). Consistent with this expectation, our diploid populations did increase in fitness more slowly than haploids over the course of the experiment, and the majority of mutations in our diploid populations fix as heterozygotes. However, we note that diploid populations do not accumulate mutations at a lower rate (and do not have a lower $\mathrm{dN} / \mathrm{dS}$ ) than our haploid MATa populations. Thus the slower rate of fitness increase in diploids could instead be partly or entirely a consequence of diminishing returns (Chou et al., 2011; Khan et al., 2011; Kryazhimskiy et al., 2014).

One way for recessive (or incompletely dominant) mutations arising in diploid populations to bypass Haldane's sieve is by loss of heterozygosity (LOH), in which a mutation is copied to the sister chromosome by mitotic recombination or wholechromosome homozygosis (Forche et al., 2011; Gerstein et al., 2014; St Charles et al., 2012). We see signatures of these LOH events across the genome in our experiment (Figure 8A). As in Marad et al. (2018) and Fisher et al. (2018), we observe certain areas of the genome with higher rates of LOH, such as the right arms of chromosomes XII and IV. These concentrations of $\mathrm{LOH}$ are likely due to some combination of selection in favor of $\mathrm{LOH}$ events and differences in the rates at which they occur. Higher rates of LOH on the right arm of chromosome XII are likely related to high levels of recombination associated with the ribosomal DNA array (Fisher et al., 2018; Marad et al., 2018), but we also see evidence that patterns of $\mathrm{LOH}$ are affected by selection for recessive beneficial mutations that would otherwise be filtered out by Haldane's sieve (as in Gerstein et al. (2014)), notably among loss-of-function mutations in the adenine pathway (Figure 3A, Figure 8A) .

As driver mutations sweep to fixation in diploids, they have the potential to bring along recessive deleterious hitchhikers (which then also fix as heterozygotes). Consistent with 

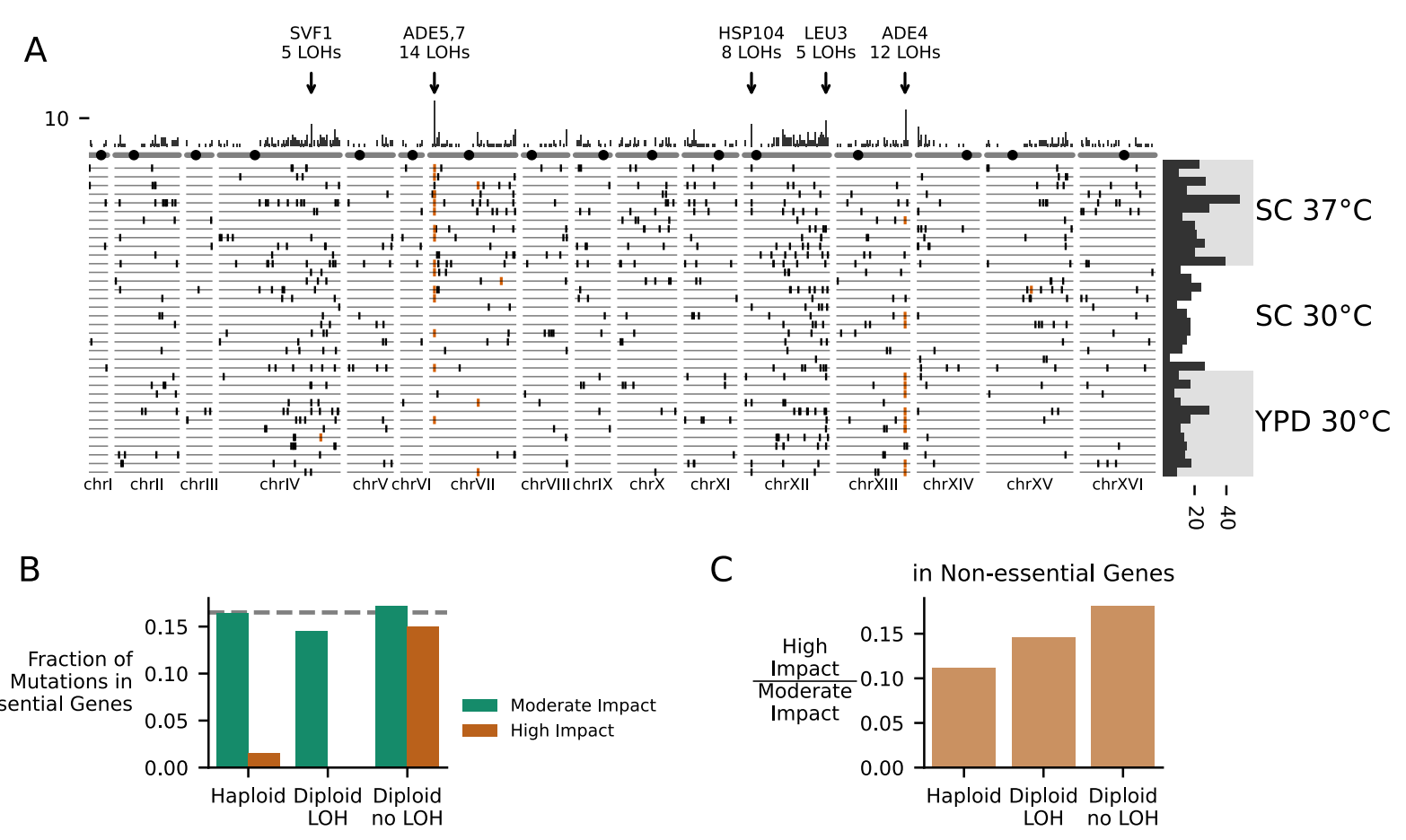

Figure 8. Patterns of Molecular Evolution and Loss of Heterozygosity in Diploids. (A) Genomic positions of all mutations that experienced loss of heterozygosity (LOH) across all diploid populations (loss of heterozygosity defined by a mutation reaching $>90 \%$ frequency). Each horizontal line represents one population, and the histogram at right represents the total number of LOH fixations in each population, with populations arranged by environment. The top histogram represents the frequency of loss of heterozygosity across the genome, and the chromosomes underneath show the centromere location with a black circle. Genes with five or more LOH fixations are annotated. (B) The fraction of fixed nonsynonymous mutations that are in essential genes, plotted for mutations fixed in haploid populations, mutations fixed homozygously in diploid populations $(\mathrm{LOH})$ and mutations fixed heterozygously in diploid populations, plotted separately for mutations annotated as high or moderate impact by SnpEff (High impact mutations are likely to cause loss-of-function). The dashed line represents the fraction of the coding genome that is in essential genes. (C) The ratio of high impact to moderate impact fixations in the same three mutation groups as in (B), for mutations in non-essential genes only.

this, we find that mutations fixed as heterozygotes in diploids include a large percentage of high impact mutations in essential genes, while mutations fixed in haploids and mutations fixed homozygously in diploids include nearly zero high impact mutations in essential genes (Figure 8B). Even in non-essential genes, mutations that fix heterozygously in diploids are more likely to be high impact mutations compared to those that fix in haploids or those that fix homozygously in diploids, again suggesting that diploids are fixing recessive deleterious mutations as heterozygotes (Figure 8C). This build-up of recessive deleterious load in diploids is expected, but takes on an interesting light in the context of the widespread loss of heterozygosity we observe. As recessive deleterious load 
accumulates in the population, it will limit the rate of LOH by making many LOH events strongly deleterious or lethal. Thus, passage through Haldane's sieve by loss of heterozygosity should become less likely as populations accumulate a substantial load of hitchhiking heterozygous mutations. However, we note that recombination with sufficient inbreeding could dramatically alter these dynamics, by continuously purging recessive deleterious load (Charlesworth and Willis, 2009).

While we hypothesize that most of the heterozygous fixations in diploids are either dominant beneficial mutations or neutral or deleterious hitchhikers, some may be overdominant beneficial mutations, which are only accessible to diploids. One strong candidate for overdominance is CCW12, which is hit preferentially in diploids (Figure 6) and in which only 2 of the 17 fixed mutations lost heterozygosity (both of these mutations are in-frame deletions of the final amino acid). In Leu et al. (2020), mutations in CCW12 were maintained in asexual diploid populations but lost in sexual populations, supporting a hypothesis of overdominance, though Leu et al. (2020) did not detect overdominance in reconstructed strains in their evolution environments. Extensive reconstructions or backcrossing will be required to understand the importance of overdominance in the evolution of our diploid populations.

\section{Loss of the 2-micron plasmid and killer phenotype}

While the mitochondria is maintained throughout evolution in all of our focal populations, we detected frequent loss or loss-of-function in three other exclusively extrachromosomal elements: the 2-micron plasmid and two double-stranded RNA components of the yeast "killer virus” toxin-antitoxin system (Schmitt and Breinig, 2002).

Following Buskirk et al. (2020) and Jerison et al. (2020), we performed halo killing assays against a sensitive strain for each of our focal populations at each sequenced timepoint (Woods and Bevan, 1968). To avoid potential inactivation of the toxin at higher temperatures in liquid media (Woods and Bevan, 1968), we conducted these assays at room temperature on agar plates. By the final timepoint, 89/90 of our focal populations lost the ability to kill a susceptible strain (Figure 9A), and this loss happened most rapidly in the high temperature environment, consistent with previous work (Jerison et al., 2020). This effect is likely due to some combination of segregation or replication failure at high temperatures (Weinstein et al., 1993) and toxin inactivation at high temperatures (Woods and Bevan, 1968). While we have not sequenced RNA viral genomes over time in our experiment, Buskirk et al. (2020) also observed widespread loss of killing ability in evolved yeast populations and determined that mutations in the K1 toxin gene were causing a loss of killing ability. While they found no evidence for a fitness benefit to the host from these loss-of-function mutations in the toxin gene, they observed that these mutations were favored in intracellular competition with other viral variants. 
A

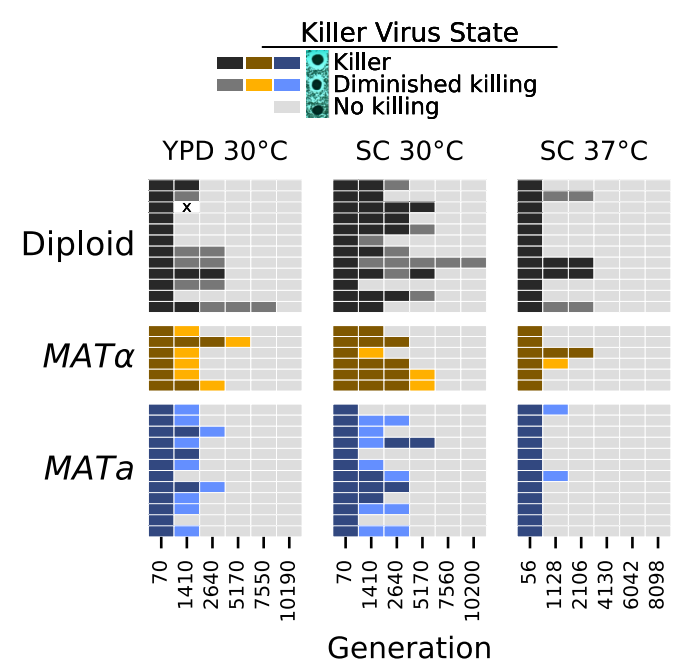

B

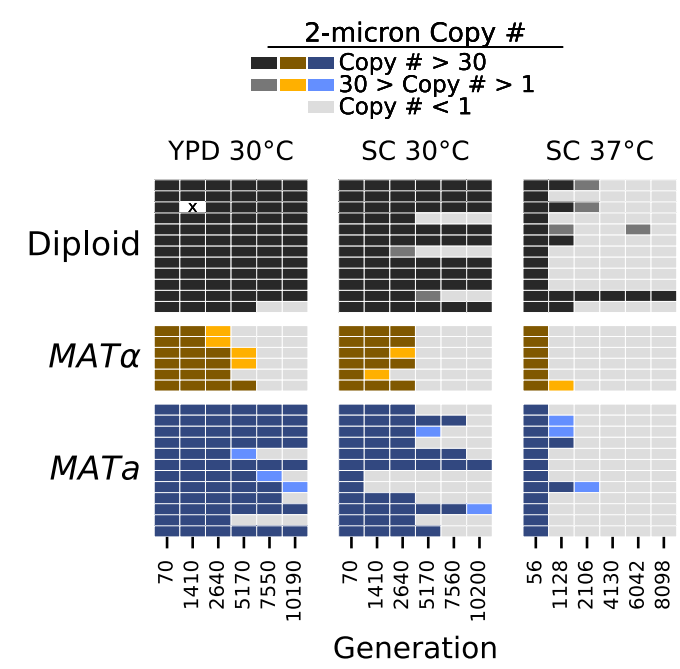

Figure 9. Loss of extrachromosomal elements. (A) Killer virus activity at each sequenced timepoint, determined by a killer assay against a sensitive strain. Each row represents one population. Examples of raw data for each qualitative phenotypic category are shown in the key, and the full raw data underlying these scores is shown in Figure 9 - figure supplement 1. (B) 2micron plasmid copy number at each sequenced timepoint. Rows represent the same populations as in $\mathbf{A}$. The $\mathrm{x}$ in a diploid population at generation 1410 marks a population we excluded due to contamination in the population during these experiments.

The 2-micron plasmid is a selfish genetic element that imposes a cost on the cell without providing any apparent benefit (Harrison et al., 2012). Because it is a DNA element, we can directly observe loss of this element in many of our populations. It appears to be lost less frequently in diploid strains, and, as with the killer phenotype, it is lost most consistently and rapidly in the high temperature environment (Figure 9B).

\section{Discussion}

Evolution experiments are as much about hypothesis generation as hypothesis testing, and work across the field has now laid out a series of hypotheses about evolution in general. No experiment can cover the breadth of biological and environmental diversity needed to fully test these hypotheses; we cannot replay all of evolution. However, a relatively consistent set of results has emerged across microbial species evolved asexually for thousands of generations in the lab (Kassen, 2014). Our results confirm many aspects of the picture drawn by previous work, with several important exceptions.

Most of our populations followed predictable fitness trajectories in which fitness increases slowed over time. This pattern was not observed, however, in some of our diploid populations, which instead increased in fitness at a slow constant rate similar to Marad et al. (2018), before experiencing significant rapid increases in fitness likely associated with individual selective sweeps (Figure 3, SC environments). Our populations show signatures 
of clonal interference, and they accumulated fixed mutations linearly through time even late in the experiment. We find only one strong case of repeatability at the level of the nucleotide change, but we observe widespread parallelism across strains and environments at the level of genes and pathways: populations predictably adapt through loss-of-function mutations in the adenine biosynthesis pathway, sterility-associated genes, and negative regulators of the Ras pathway.

We do not observe two phenomena that results from the LTEE had previously suggested might be common: the fixation of mutator alleles that dramatically increase mutation rates, and the spontaneous emergence of long-term quasi-stable coexistence between competing lineages. The reasons for these differences remain unclear. In part, we may not observe these phenomena simply because of the shorter timescale of our experiment. However, we note that within the first 10,000 generations of the LTEE, 4 of the 12 populations fix mutator alleles, and 3 of the 12 populations have coexisting lineages detectable from sequencing data. Instead, the lack of mutator lineages may stem from a difference in the rate at which mutators arise or a different balance between the relative importance of beneficial and deleterious mutations (or differences in other population genetic parameters such as the population size or wild-type mutation rate) that leads to less indirect selection for mutators (Good and Desai, 2016). The difference in how commonly coexistence emerges is similarly unclear. Our strains and environments may simply lack the metabolic pathway architecture to produce cross-feeding or other interactions that could be the basis for coexistence. Regardless of the reasons for these differences, our results suggest that the evolution of mutation rates and of stable ecological interactions may not be as general or widespread as the LTEE has suggested, and may instead vary substantially based on differences in the organisms or details of the environmental conditions.

As the longest running evolution experiment in yeast, this project provides a window into how dominance and loss of heterozygosity can affect the dynamics of adaptation in diploids. Our diploid populations appear to carry substantial recessive deleterious load (Figure 8B-C) and may carry beneficial overdominant mutations, but future studies involving genetic reconstructions or backcrossing will be needed to fully characterize these effects. We also observe widespread loss of heterozygosity. The dynamics of mutations in the adenine biosynthesis pathway provides a particularly interesting example of both how Haldane's sieve slows adaptation in diploids and how diploids can bypass the sieve by loss of heterozygosity. At some point during the experiment, most of our diploid populations homozygously fix a loss-of-function mutation upstream in the pathway, which eliminates the deleterious toxic intermediate produced as a result of the ancestral ade2-1 mutation. While the rate of loss of heterozygosity was high enough to produce these genotypes and expose them to selection, it appears to have been a limiting factor; haploids typically fixed these mutations earlier in the experiment (Figure 3 - figure supplements 2-10). Haldane's "speedcheck" here slowed adaptation but also provided diploid populations with more time to search for the single-codon target of the (highly 
beneficial and apparently dominant) ade2-1 reversion, and indeed, 4/6 populations with this mutation in our experiment are diploids.

Perhaps the most important product of microbial evolution experiments is a base of intuition for understanding how the interactions between different evolutionary forces determine the dynamics and outcomes of genotypic and phenotypic evolution. The extent to which this base of intuition can be generalized across systems and scales -- ranging from specific protein complexes to human pathogens to entire clades of sexually reproducing species -- is an important set of largely unanswered questions. However, laboratory microbial evolution experiments have provided basic expectations to compare against, and have highlighted a collection of phenomena that can sometimes play a major role in adaptation. Our results here reinforce the conclusion that long-term adaptation to a constant environment can be characterized by widespread clonal interference, contingency, and steady molecular evolution even as fitness increases slow down over time. They also highlight the role of dominance and loss of heterozygosity in diploid evolution. However, our work also calls into question the generality of conclusions about the importance of the evolution of mutation rates or stable coexistence. As our populations continue to evolve, further analysis of our experiment and of other complementary studies will further broaden our understanding of the processes that determine the rate, predictability, and molecular basis of evolution. 


\section{Materials and Methods}

\section{Strains}

The two haploid strains used for this study are MJM361, which has genotype MATa,

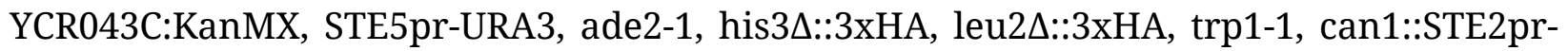

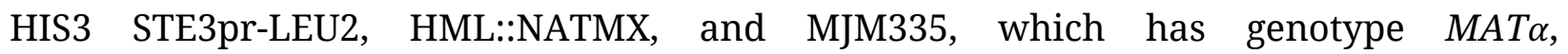
YCR043C:HGHB, STE5pr-URA3, ade2-1, his30::3xHA, leu2A::3xHA, trp1-1, can1::STE2prHIS3 STE3pr-LEU2, HMR::NATMX. We created MJM361 and MJM335 by knocking out HML or HMR with the NatMX cassette in MJM64 and MJM36 respectively (McDonald et al., 2016). The diploid strain used in this study, MJM102, is a cross of MJM64 and MJM36.

\section{Culture conditions}

We propagated all populations in $128 \mu \mathrm{L}$ of media in unshaken flat-bottom polypropylene 96-well plates (VWR \#82050-786). For one environment, we used rich YPD media (1\% Bacto yeast extract (VWR \#90000-726), 2\% Bacto peptone (VWR \#90000-368), 2\% dextrose (VWR $\# 90000-904)$ ) and grew populations at $30^{\circ} \mathrm{C}$. For the other two environments, we used synthetic complete (SC) media (0.671\% YNB with nitrogen (Sunrise Science \#1501-250), $0.2 \%$ SC (Sunrise Science \# 1300-030), $2 \%$ dextrose) and grew populations at $30^{\circ} \mathrm{C}$ or $37^{\circ} \mathrm{C}$. All media was supplemented with $100 \mu \mathrm{g} / \mathrm{ml}$ ampicillin and $25 \mu \mathrm{g} / \mathrm{ml}$ tetracycline. We performed daily $1: 2^{10}$ dilutions of populations in YPD $30^{\circ} \mathrm{C}$ and SC $30^{\circ} \mathrm{C}$ and daily $1: 2^{8}$ dilutions of populations in SC $37^{\circ} \mathrm{C}$ using a BiomekFXp robot (Beckman Coulter). Before dilution, we resuspended cultures by shaking at $1200 \mathrm{rpm}$ for 2 minutes, and after dilution we shook the new plates at $1200 \mathrm{rpm}$ for 1 minute, both on a Titramax 100 plate shaker (Heidolph Instruments). After each transfer, the tips (VWR \#89204-794) used to dilute cultures were washed with water (to wash out cells) and 100\% ethanol (to lyse residual cells), left to dry overnight, and reused in culture propagation. The 96-well microplates used to maintain populations were bleached (to lyse cells), washed with distilled water, and autoclaved $\left(121^{\circ} \mathrm{C}, 30 \mathrm{mins}\right)$ before being reused. Every 7 days, we froze aliquots of all populations in $27 \%$ glycerol (final concentration) at $-80^{\circ} \mathrm{C}$. To monitor for contamination, 6 well-spaced wells in each environment were intentionally left "blank" at the start of the experiment (i.e. they contained only media and no cells). At several timepoints during the evolution we noticed contamination in the previously blank wells of our 96 well plates. During instances of contamination, we unfroze all populations from an older glycerol archive and inoculated $4 \mu \mathrm{L}$ directly into $124 \mu \mathrm{L}$ of the appropriate media for each environment. A record with notes on the evolution is available in Supplementary file 1.

\section{Population loss and cross-contamination}

Over the course of this experiment, we periodically screened our populations for drug resistance (Hygromycin, G-418, and ClonNat) in order to detect cross-contamination. Using these checks, we observed multiple instances of cross-contamination in the YPD $30^{\circ} \mathrm{C}$ environment from other yeast species which were part of a concurrent evolution experiment. These events were likely due to mistakes during our tip washing or media filling procedures, which were more carefully controlled later in the experiment. As 
described above for the case of outside contamination, if we observed cross-contamination shortly after it occurred, we restarted the experiment from a previously frozen timepoint. In several cases we failed to recognize cross-contamination until thousands of generations had passed, so we excluded these populations from our analysis (Figure 1 - figure supplement 1 lists these populations). While it is possible that cross-contamination occurred more frequently than just the cases we observed, the sequencing data for focal populations suggests otherwise (fixed mutations remained fixed in all populations for the duration of the experiment). Due to a combination of errors in Biomek pipetting and evaporation (especially in the $37^{\circ} \mathrm{C}$ environment), we also lost several populations over the course of the experiment (the wells became blanks); these are also excluded from our analysis, and are listed in Figure 1 - figure supplement 1.

\section{Fitness assays}

In order to assess competitive fitness using a consistent reference for each environment, we isolated clones at various generations from an arbitrarily chosen evolving diploid population in $\mathrm{YPD} 30^{\circ} \mathrm{C}$ (P1G09). We looked for clones that had fitnesses intermediate between the ancestral strains and evolved strains in each environment, and tagged these clones by inserting a yNatMX cassette and GFP (pRPL39::eGFP::tADH) into an intergenic region (chromosome VII, position 649234) that was previously used as a neutral insertion site control in Johnson et al. (2019). This produced the reference strains used for fitness assays in YPD $30^{\circ} \mathrm{C}$ and SC $30^{\circ} \mathrm{C}(2490 \mathrm{~A}-\mathrm{GFP} 1)$, and SC $37^{\circ} \mathrm{C}(11470 \mathrm{~A}-\mathrm{GFP} 1)$.

Fitness assays were performed as described previously (Lang et al., 2011). Briefly, we unfroze populations and a reference strain from glycerol stocks, allowed them to grow in their evolution environment for one full growth cycle, and then mixed the populations with the reference strain in equal proportions. We then maintained these mixed populations for 3 daily growth cycles, as described above. At each transfer, we diluted cells from each well into PBS and used flow cytometry (Fortessa and LSRII, BD Biosciences) to measure the ratio of the two competing types, counting approximately 10,000-40,000 cells for each measurement.

To get fitness measurements for each population-timepoint, we first calculated the frequency of fluorescent reference cells in each sample by gating our flow cytometry data to separate the fluorescent cells. Because a small percentage of reference cells do not fluoresce, we estimated this percentage from six wells that only contained the reference in each environment and used these values to correct the reference frequency in all other wells. We then calculated the fitness of each population-timepoint as the slope of the natural log of the ratio between the frequencies of the non-reference and reference cell populations over time (timepoints with reference frequency under $5 \%$ or over $95 \%$ were excluded). After taking the mean of fitness measurements from two replicates, we corrected for batch effects in our assays by subtracting the mean fitness measured for an unlabeled reference (2490A) in the same fitness assay. 
Because the ancestral genotypes have a strongly deleterious mutation in the adenine biosynthesis pathway and haploids very quickly fix strongly beneficial suppressor mutations, it was difficult to measure ancestral fitness in some cases; we sometimes observed changes in fitness during the fitness assay even when using clones from generation zero (which had been grown prior to and after freezing glycerol stocks). In all but one environment-strain combination, we were able to identify populations without any nonsynonymous mutations detected from our sequencing data the first timepoint (generation 70 for $\mathrm{YPD} 30^{\circ} \mathrm{C}$ and SC $30^{\circ} \mathrm{C}$, generation 56 for SC $37^{\circ} \mathrm{C}$ ), so we used the median of the fitness measured among these populations at the first timepoint to define ancestral fitness. All MATa populations in YPD $30^{\circ} \mathrm{C}$ had nonsynonymous mutations present (and often fixed) at generation 70, but one unsequenced population had a significantly lower generation 70 fitness than all others (similar to the one $M A T \alpha$ population in YPD $30^{\circ} \mathrm{C}$ with no-nonsynonymous mutations at generation 70), so we use the fitness estimated at generation 70 for that population as our ancestral fitness for MATa populations in YPD.

\section{Whole-genome sequencing}

For each of the three environments, we selected 30 focal populations: 12 diploid populations, 12 MATa populations, and 6 MAT $\alpha$ populations. We chose these populations randomly after excluding populations in wells along the edge of the plate (which we have had the most problems with losing due to evaporation or pipetting errors) and populations where we had detected cross-contamination. We performed whole-genome, wholepopulation sequencing on each of these populations at 6 timepoints. After unfreezing populations as described above, we transferred each of our focal populations into 5 replicate wells in their evolution environment, let them grow for 24 hours, and then pelleted $\sim 0.5 \mathrm{~mL}$ of cells. We used a DNA extraction protocol based on the "BOMB gDNA extraction using GITC lysis” from Oberacker et al. (2019). Briefly, we resuspended the cell pellets in $50 \mu \mathrm{L}$ of zymolyase buffer ( $5 \mathrm{mg} / \mathrm{mL}$ Zymolyase 20T (Nacalai Tesque), 1M Sorbitol, 100mM Sodium Phosphate pH 7.4, 10mM EDTA, 0.5\% 3-(N,N-Dimethylmyristylammonio)propanesulfonate (Sigma, T7763), 200 $\mu \mathrm{g} / \mathrm{mL}$ RNAse A, and 20mM DTT) (Nguyen Ba et al., 2019) and incubated the suspension at $37^{\circ} \mathrm{C}$ for 1 hour. Subsequently, we added $85 \mu \mathrm{L}$ of a modified BOMB buffer (4M guanidinium-isothiocyanate (Goldbio G-210-500), 50 mM Tris$\mathrm{HCl} \mathrm{pH} \mathrm{8,} 20 \mathrm{mM}$ EDTA) and then $115 \mu \mathrm{L}$ of isopropanol (VWR\# BDH1133-4LP), mixing by pipetting for 3 minutes after each addition. We then added $20 \mu \mathrm{L}$ of Zymo Research MagBinding beads to bind DNA, mixed for 3 minutes by pipetting, separated beads from the solution using a Magnum FLX 96-well magnetic separation rack (Alpaqua), and removed the supernatant. We washed the beads with $400 \mu \mathrm{L}$ of isopropanol and twice with $300 \mu \mathrm{L}$ of $80 \%$ ethanol. Finally, we added $75 \mu \mathrm{L}$ of sterile water to the beads and mixed by pipetting for 3 minutes. Finally, we separated beads from solution and transferred $44 \mu \mathrm{L}$ of the supernatant (containing the DNA) into a new 96-well PCR plate (Bio-Rad HSP9631) for library preparation. This entire process was carried out on a BiomekFXp robot (Beckman Coulter). 
Sequencing libraries were prepared using a Nextera (Illumina) kit as previously described (Baym et al., 2015), but with 3 additional PCR cycles for a total of 16, and with a two-sided bead-based size selection after PCR (we used either $0.5 / 0.7 \mathrm{X}$ or $0.55 / 0.65 \mathrm{X}$ bead buffer ratios with PCRClean DX Magnetic Beads (Aline)). Libraries were sequenced to an average depth of 20-fold (haploids) or 40-fold (diploids) coverage using a Nextseq 500 (Illumina).

\section{Sequencing analysis}

We trimmed Illumina reads with NGmerge version 0.2 (Gaspar, 2018), aligned all the firsttimepoint samples to a SNP-corrected W303 genome (Lang et al., 2013) using BWA version 0.7.15 (Li, 2013), and marked duplicate reads with Picard version 2.9.0 (http://broadinstitute.github.io/picard). We used samtools (Li et al., 2009) to merge these alignments and then used Pilon version 1.23 (Walker et al., 2014) to create a new reference genome that is corrected for additional SNPs present in the ancestral strains. We then repeated this process until the marking duplicate reads step for all samples using this new reference and called variants using GATK version 4.1.3.0 (McKenna et al., 2010), specifically using HaplotypeCaller, GenomicsDBImport, and GenotypeGVCFs with heterozygosity set to 0.005. We annotated these variants using SnpEff version 4.3T (Cingolani et al., 2012), and split multi-allelic records into individual records.

We extracted allele depths for each variant to determine the number of reads supporting the reference and alternate alleles at each site. We then filtered variants based on these read counts. We first excluded mutations with less than 5 reads representing the alternate allele across all timepoints. To create this filtered list of variants present in each population, we required that mutations pass at least one of these two criteria:

1) The total alternate-allele reads across all timepoints for the population in question is more than $90 \%$ of the total alternate-allele reads across all populations and all timepoints.

\section{OR}

2) (At least two timepoints have at least 5 reads supporting the alternate allele) AND (The total alternate-allele reads across all timepoints for the population in question is more than $90 \%$ of the total alternate-allele reads across all populations at only the first timepoint).

The first criterion addresses if a mutation is unique to a single population, which provides strong evidence that it is not a common sequencing or alignment error. However, we do not want to exclude the possibility of parallelism at the nucleotide level, so we include the second criterion as a more lenient way to exclude these types of errors while not requiring uniqueness. Some small number of sequencing or alignment errors will pass these filters, so we emphasize that this is only a lenient first step, and that our analysis of parallelism and contingency relies on also observing fixation. 
We simplify our SnpEff annotations to indicate one of five types of mutation; in order of decreasing putative effect they are indel, nonsense, missense, synonymous, or noncoding. For mutations with multiple annotations, we assign the mutation type with the largest putative effect. To test if some nearby mutations are part of a single mutational event, we perform Fisher's exact test on the alternate and reference allele counts at each timepoint for mutations within $25 \mathrm{bp}$ of each other. If two mutations have no significant differences detected at the $P<0.01$ level for any timepoint, we label them as part of the same "mutation group," and they are counted as one mutation in subsequent analysis. We define mutations as "present" at a particular timepoint if they have coverage of at least 5X and are at greater than or equal to 0.1 frequency. We define mutations as "fixed" at a particular timepoint if they have coverage of at least 5X, are at greater than or equal to a frequency of $40 \%$ (diploids) or $90 \%$ (haploids), and do not drop below these thresholds while still at $>=5 \mathrm{X}$ coverage at a later timepoint. If a mutation is called fixed at one timepoint, it is automatically called fixed at later timepoints, even if they have less than 5X coverage. Using the same rules, we also call loss of heterozygosity of a mutation in diploids using a frequency threshold of $90 \%$. We exclude mutations called in the 2-micron plasmid from further analysis since most populations lose this plasmid during evolution and variation in coverage, and misalignments can easily produce false mutation calls in these cases. We also exclude mutations in the telomeres, where alignment errors and repetitive regions make mutation calling difficult.

\section{Structural variant / copy number variant analysis}

We use LUMPY and smoove (https://github.com/brentp/smoove) to call structural variants in our sequencing data (Layer et al. 2014). All structural variants called are listed in the processed variant call files for each population included in Supplementary file 2. In addition, we use a custom pipeline to identify putative copy number variants (CNVs) in our data. We use samtools-depth to calculate per-site depth from our bam files, and then calculate the average depth in non-overlapping $500 \mathrm{bp}$ windows along the genome. We calculate the median window-depth across the entire genome for each sample and divide all window-depths by this value to get "relative depth." To account for regions that are at a different copy number in our ancestral strains, we calculate the average relative depth at the first sequenced timepoint for each window (and for each strain). We divide the relative depth in our data by these values to get "standardized depth." Windows with a relative depth less than 0.25 at the first timepoint are excluded from analysis. For each chromosome in each sample, we use a simple, untrained HMM to detect tracts of standardized depth that deviate from the expectation of 1 . We allow states $0,0.5,1,1.5,2$, 3 , and 4, with variances equal to the calculated variance in standardized depth multiplied by the state (except for state 0 , where we use the calculated variance multiplied by 0.5), initial probabilities of $1 \%$ for each non-1 state (94\% for state 1$)$, and transition matrix probabilities of $.01 \%$ for all non-diagonal entries (99.94\% along the diagonal). This is a rough detection method, but it succeeds in identifying putative CNVs, which we then subject to a filtering process. First, we merge CNV records across timepoints if they cover the same region. Next, we exclude CNVs in telomeric regions, CNVs found in only one 
timepoint, and CNVs that are less than 4 windows (2kb) long. Finally, we manually inspect our structural variant and CNV calls together using a modified version of Samplot (https://github.com/ryanlayer/samplot) to create a list of confirmed copy number variants in our populations (Supplementary file 3, structural variants detected in at least two different populations are listed in Figure 3 - figure supplement 11). During this analysis, we noticed two regions with high copy-number that experienced copy-number changes in many populations: one associated with the CUP1 tandem array and one associated with the ribosomal DNA tandem array. We excluded these regions from the above analysis and show their copy number changes in every population in Figure 3 - figure supplement 12.

\section{Analysis of multi-hit genes}

To look for evidence of parallelism and contingency in our data, we focus on nonsynonymous mutations in genes (we consider all open reading frames to be genes for this analysis) that are fixed at the final timepoint. We define the multiplicity as the number of hits multiplied by the number of possible nonsynonymous mutations for that gene, scaled by the mean number of possible nonsynonymous mutations across all genes. In our null model, the hits for each population (the number of hits from our data) are randomly assigned to genes from the complete set of 6579 annotated open reading frames in $S$. cerevisiae, weighted by the number of possible nonsynonymous mutations in each gene. We simulate these random draws 1,000 times to generate null distributions for gene multiplicity and number of unique populations in which a gene has at least one hit. To look for parallelism at the codon level, we randomize the location of each nonsynonymous fixed mutation in the gene in which it occurred and then count the number of populations with simulated hits at each amino acid position. We repeat this process ten times to build a null distribution and compare it to the empirical distribution of populations hit for each amino acid position (Figure 5C).

Next, we move away from using multiplicity, since we know that selection is playing a large role and mutation rate is not completely limiting adaptive dynamics (clonal interference is observed in our sequencing data). This means that as we look to identify common targets of selection, we will treat the probability of a hit in any gene where we have observed at least one hit as equally likely (instead of weighting by the number of possible nonsynonymous mutations). To this end, we define multi-hit genes as those with hits in at least six populations. Based on the simulations above, a gene has a $P=0.02$ chance of being hit in at least six populations. While this is a lenient cutoff that will produce a number of false positives, we see a large excess of genes with hits in greater than six populations in our data (Figure 5B). To look for functional patterns in our mutation data, we performed GO-Term Enrichment on the set of multi-hit genes analysis using the GOATOOLS python library (Klopfenstein et al., 2018). The enrichments that were significant after Benjamini-Hochberg multiple hypothesis testing correction are listed in Figure 6 - figure supplement 2. 
We define essential genes based on data from the yeast gene deletion collection (Giaever et al., 2002; Liu et al., 2015). The set of essential genes used in Figure 8 are those classified as non-evolvable in Liu et al. (2015).

\section{Multi-hit gene enrichment by strain and/or environment}

Within a given set of populations, we model the probability of a multi-hit gene $i$ being associated with a fixation event based on $\tilde{\mathrm{N}}_{i}$, the number of populations with a mutation fixed in gene $i$ plus a 0.1 pseudocount (to avoid zero probabilities), and $M$, the total number of gene hits across all populations in the set (we ignore when a gene is hit multiple times in the same population for this probability calculation). We model the probability a gene is hit in population $j$ based on the total number of gene hits in population $j, M_{j}$ :

$P($ Gene $i$ not hit in pop $j)=P\left(h_{i j}=0\right)=\left(1-\left(\tilde{\mathrm{N}}_{i} / M\right)\right)^{M_{j}}$,

$P($ Gene $i$ hit in pop $j)=P\left(h_{i j}=0\right)=1-\left(1-\left(\tilde{\mathrm{N}}_{i} / M\right)\right)^{M_{j}}$.

To test whether genes are disproportionately mutated in different strain backgrounds (MATa, MAT $\alpha$, diploid) or different environments, we compare four models that use different sets of populations to compute $P\left(h_{i j}\right)$ :

1) $P\left(h_{i j}\right)$ is calculated using the entire set of populations (so that there is only one $P\left(h_{i j}\right)$ for each multi-hit gene)

2) $P\left(h_{i j}\right)$ is calculated separately for each strain background (so that there are three $P\left(h_{i j}\right)$ for each multi-hit gene, one for each strain)

3) $P\left(h_{i j}\right)$ is calculated separately for each environment (so that there are three $P\left(h_{i j}\right)$ for each multi-hit gene, one for each environment)

4) $P\left(h_{i j}\right)$ is calculated separately for each environment-strain combination (so that there are nine $P\left(h_{i j}\right)$ for each multi-hit gene, one for each environment-strain combination)

For each multi-hit gene, we calculate the log-likelihood of the data under each model and calculate log-likelihood ratios between model 1 and each of the other three models. We then create 10,000 null datasets by drawing values from the probabilities defined in model 1 and compute log-likelihood ratios for these simulated data. To define significant effects (at a $P<0.05$ level), we compare our log-likelihood ratios to distributions of log-likelihood ratios from these null datasets and correct for multiple hypothesis testing using a Benjamini-Hochberg correction. If multiple models are significantly better than model 1, we use the Akaike information criterion (AIC) to determine the model that best explains the data. Data on multi-hit genes and these statistical tests are available in in Supplementary file 4.

\section{Mutual information analyses}

Next, we investigate whether the fixation of a mutation in any of our multi-hit genes is dependent on fixation of a mutation in another multi-hit gene. For each environment- 
strain combination, we calculate mutual information between all multi-hit genes as described in Fisher et al. (2019). Because we separate our data into sets of populations with a shared environment and strain background, our data contain many cases where a gene is hit zero times, which inflates the sensitivity to the pseudocount used in Fisher et al. (2019). To avoid this issue, we set the mutual information between two genes to zero if either of the genes has no mutations in a given environment-strain combination. We sum the mutual information values for each pair of genes across the 9 possible environmentstrain combinations, and record the total mutual information ( $M I_{\text {tot }}$, the sum of $M I$ values across all possible gene pairs) and the maximum mutual information between any two genes $\left(M I_{\max }\right)$.

Next, we compare these results to simulated datasets. To avoid mistaking an environment or strain effect for an association between genes, we use separate $P\left(h_{i j} \mid e\right)$ for each environment-strain combination $e$, as in model 4 above. We simplify our $P\left(h_{i j} \mid e\right)$ expression to $P\left(h_{i} \mid e\right)$ here by treating populations as exchangeable (note that the number of fixed mutations in each environment-strain combination is not highly variable (Figure 3B)), so that $P\left(h_{i}=0 \mid e\right)=1-\left(N_{i} / N_{t o t, e}\right)$ and $P\left(h_{i}=1 \mid e\right)=N_{i} / N_{t o t, e}$, where $N_{\text {tot, } e}$ is the total number of populations in environment-strain combination $e$. We create 10,000 null datasets by drawing from $P\left(h_{i} \mid e\right)$, and calculate mutual information as described above to build null distributions for $M I_{\text {tot }}$ and $M I_{\max }$.

The results are plotted in Figure 7 - figure supplement 1. While our $M I_{\text {tot }}$ for our data is higher than in simulated datasets $(P=0.03), M I_{\max }$ for our data lies well within the range of simulated data, so we cannot detect any specific examples of contingency. As in Fisher et al. (2019), we test the robustness of our results to choices of the pseudocount $\varepsilon M$ between 0.1 and 2 (the value used above was 1), and find that it does not qualitatively change our results.

\section{Over-/under- dispersion analysis}

Following Good et al. (2017), we looked for statistical patterns of contingency by comparing the dispersion configurations for genes with simulated data. For each environment-strain combination, we record the number of times each gene is hit and the number of populations in which it is hit. We also simulate distributing these hits across populations by multinomial draws weighted by the number of hits in each population. We run this simulation, for each possible number of hits (up to the maximum observed), 10,000 times for each environment-strain combination. For each number of hits, we compute the probability of those hits being distributed among each possible number of populations for both our data and the simulated data. We compute the "excess probability" in our data by subtracting the simulated probability from the data probability. The results are plotted in Figure 7 - figure supplement 2A. We repeat this process with mutations that are detected but do not fix included (Figure 7 - figure supplement 2B). Red squares along the diagonal suggest that the mutations are overdispersed, meaning that nonsynonymous mutations are less likely to fix multiple times in the same population than we would expect 
by chance. As in Good et al. (2017), we quantify this observation of overdispersion by showing that mutations have less "missed opportunities" than we would expect by chance (Figure 7 - figure supplement 2).

\section{Killer phenotype assays}

To assay for the killer phenotype, we used a modified halo killing assay (Woods and Bevan, 1968). First, we plated a $150 \mu \mathrm{L}$ of 1:100 diluted saturated culture of a sensitive strain (YAN563) on a single-well (VWR \#46600-638) methylene blue agar plate (20 g/L peptone, 10 $\mathrm{g} / \mathrm{L}$ yeast extract, $20 \mathrm{~g} / \mathrm{L}$ citric acid monohydrate, $30 \mathrm{mg} / \mathrm{L}$ methylene blue, $10 \mathrm{~g} / \mathrm{L} \mathrm{K2HPO4,}$ $20 \mathrm{~g} / \mathrm{L}$ dextrose, $15 \mathrm{~g} / \mathrm{L}$ noble agar). We then used the Biomek Fxp Liquid Handler to spot $3.5 \mu \mathrm{L}$ of saturated culture of each of our focal populations onto this lawn. We designed a Biomek protocol that uses a deck spring attachment ("Alpillo” from Alpaqua) to make sure that the tips contacted the agar but did not pierce the agar layer during this step. After 2-3 days of incubation at room temperature, we scanned the plates. We scored each population-timepoint as "Killer," "Diminished Killing," or "No Killing," based on the size of the zone of inhibition (halo) around the spot (see Figure 9A for examples of each category, and Figure 9 - figure supplement 1 for the underlying images). The sensitive strain used (YAN563) is a cross between YAN457 (MATa, his3 $\Delta 1$, ura3 $\Delta 0$, leu2 $\Delta 0$, lys $2 \Delta 0$, RME1pr::ins308A, ycr043c $\Delta 0:: N a t M X$, can1::RPL39pr_ymGFP_Ste2pr_SpHIS5_Ste3pr_LEU2, derived from BY4742) and YAN433 (MATa, his3 $\Delta 1$, ura3 $\Delta 0$, leu2 $\Delta 0$, lys2 $\Delta 0$, RME1pr::ins-308A, ycr043c $\Delta 0:: H p h M X 4$, can1::RPL39pr_ymCherry_Ste2pr_SpHIS5_Ste3pr_LEU2, derived from BY4742).

\section{Ploidy assays}

To investigate whether any of our focal populations had changed ploidy during the course of the experiment, we measured the DNA content of clones isolated from each focal population at the final timepoint. We isolated 1-2 clones from each focal population and measured DNA content using a nucleic acid stain as described previously in Jerison et al. (2020), but with minor modifications. Briefly, we diluted $4 \mu \mathrm{L}$ of saturated cultures from each clone (grown in YPD) into $120 \mu \mathrm{L}$ of water in a 96-well plate, centrifuged the plate, removed the supernatant, resuspended in $50 \mu \mathrm{L}$ water, added $100 \mu \mathrm{L}$ of ethanol and pipetted slowly to mix, and incubated at room temperature for 1 hour. Next, we centrifuged the plate, removed the supernatant, let dry for $\sim 5$ minutes, resuspended in 65 $\mu \mathrm{L}$ RNase solution ( $2 \mathrm{mg} / \mathrm{ml}$ RNase in $10 \mathrm{mM}$ Tris-HCl, $\mathrm{pH} 8.0$ and $15 \mathrm{mM} \mathrm{NaCl}$ ), and incubated at $37^{\circ} \mathrm{C}$ for 2 hours. We then added $65 \mu \mathrm{L}$ of $2 \mu \mathrm{M}$ Sytox Green (Thermo Fisher Scientific S7020), covered the plates in aluminum foil, and shook on a Titramax 100 plate shaker (Heidolph Instruments) for approximately 45 minutes at room temperature. We measured DNA content using a linear FITC channel on a Fortessa flow cytometer (BD Biosciences). FITC histograms are shown and described in Figure 8 - figure supplement 1. 


\section{Determining the mutation responsible for a higher mutation rate in MATa populations}

To investigate the putative higher mutation rate in the MATa populations in our experiment, we examined a list of mutations that differentiate our MATa ancestor and our $M A T \alpha$ ancestor (available in Supplementary file 5), and identified a putative causative mutation: a missense mutation at a conserved residue in TSA1 (G146S, nucleotide mutation: G->A at bp 436). TSA1 encodes thioredoxin peroxidase, which is involved in eliminating reactive oxygen species that can cause DNA damage, and previous work has shown that deleting the gene causes an increase in mutation rate (Huang et al., 2003). As we would expect, this mutation is heterozygous in the diploid ancestor.

We reconstructed the TSA1 G146S mutation by Delitto Perfetto in the S288C background (Storici and Resnick, 2006). Briefly, we transformed BY4741 with a KlURA3-KanMX4 cassette, knocking out the whole TSA1 gene, thus creating YAN727. We then removed the cassette with a PCR amplified TSA1 fragment containing the G146S mutation, selecting on 5-FOA and the absence of G418 resistance, thus creating YAN728. The presence of the mutation was then confirmed by Sanger sequencing. We used BY4741 for these reconstructions because our $M A T \alpha$ ancestor has a functional URA3 (under the STE5 promoter), making it more difficult to create this type of reconstruction.

We performed fluctuation assays on each of the three strains as previously described (Lang and Murray, 2008). Briefly, we inoculated a colony from each strain into SC, grew overnight, diluted 1/10,000 and split into $100 \mu \mathrm{L}$ aliquots in all wells of a 96-well plate, sealed the plate with aluminum foil, and incubated at $30^{\circ} \mathrm{C}$ without shaking for 48 hours. We combined the 8 wells from column 2 of each plate and used the pooled culture to measure cell density on a Coulter Counter Z2 (Beckman Coulter). We spotted the entire volume of each of the other 88 wells on CSM-Arg (Sunrise Scientific) plates supplemented with $100 \mathrm{mg} / \mathrm{L}$ L-canavanine (Sigma-Aldrich, St. Louis). Plates were allowed to dry overnight at room temperature, incubated at $30^{\circ} \mathrm{C}$ for 36 hours, then left at room temperature for 12 hours before counting and scanning. We attempted to count all colonies $>0.25 \mathrm{~mm}$ in size in each spotted culture, but note that our estimates of counts $>50$ are approximate due to overlapping colonies (counts available in Supplementary file 5).

We used the Ma-Sandri-Sarkar Maximum Likelihood Estimator (Sarkar et al. 1992), implemented in python (https://github.com/bondarevts/flucalc), to measure the mutation rate at the CAN1 locus (Radchenko et al., 2018; Sarkar et al., 1992). As noted in Lang and Murray (2008), there is likely limited postplating growth of sensitive yeast at this Canavanine concentration, leading to the deviations from the expected Luria-Delbruck distribution in Figure 3 - figure supplement 13. Despite this complication, we can easily see that the TSA1 mutation causes a $\sim 5$-fold increase in mutation rate over the BY4741 background, and the TSA1 deletion causes an $\sim 8$-fold increase in mutation rate over the BY4741 background (Figure 3 - figure supplement 13). In our case, the important result is simply that the TSA1 G146S mutation causes an increase in mutation rate, consistent with 
the hypothesis that it underlies the higher number of fixed mutations in MATa populations in our experiment.

\section{Acknowledgements}

We thank Andrew Murray, Nina Benites, Yi Chen, and members of the Desai lab for helpful comments on the manuscript. We thank the Northwest building staff, in particular Francisco Gonzalez, and the Bauer Core staff, without whom we could not have done this work. This work was supported by NSF Graduate Research Fellowships (MSJ, ERJ, KK, and KRL), the NSF-Simons Center for Mathematical and Statistical Analysis of Biology at Harvard University Grant DMS-1764269 (KRL), the Harvard Program for Research in Science and Engineering (JG), the NDSEG Fellowship Program (CWB), the Fannie \& John Hertz Foundation Graduate Fellowship Award (KRL), the Boston Bangalore Biosciences Beginnings Program from DBT, India (RP), the ARC Grant FT 170100441 (MJM), the NSERC (ANNB), Simons Foundation Grant 376196 (MMD), NSF Grant PHY-1914916 (MMD), and NIH Grant R01 GM104239 (MMD). Computational work was performed on the Cannon cluster supported by the Research Computing Group at Harvard University.

\section{Data and code accessibility}

Raw sequencing data is available on NCBI: https://www.ncbi.nlm.nih.gov/bioproject/668346

All code used in this project is available on GitHub: https:/github.com/mjohnson11/VLTE PIPELINES 


\section{References}

Bailey SF, Rodrigue N, Kassen R. 2015. The effect of selection environment on the probability of parallel evolution. Molecular Biology and Evolution 32:1436-1448.

Barrick JE, Yu DS, Yoon SH, Jeong H, Oh TK, Schneider D, Lenski RE, Kim JF. 2009. Genome evolution and adaptation in a long-term experiment with Escherichia coli. Nature 461:1243-1247.

Baym M, Kryazhimskiy S, Lieberman TD, Chung H, Desai MM, Kishony R. 2015. Inexpensive multiplexed library preparation for megabase-sized genomes. PLoS One 10:e0128036.

Behringer MG, Choi BI, Miller SF, Doak TG, Karty JA, Guo W, Lynch M. 2018. Escherichia coli cultures maintain stable subpopulation structure during long-term evolution. PNAS 115:E4642-4650.

Blount ZD, Barrick JE, Davidson CJ, Lenski RE. 2012. Genomic analysis of a key innovation in an experimental Escherichia coli population. Nature 489:513-518.

Buskirk SW, Rokes AB, Lang GI. 2020. Adaptive evolution of nontransitive fitness in yeast. bioRxiv. doi:10.1101/700302

Charlesworth D, Willis JH. 2009. The genetics of inbreeding depression. Nature Reviews Genetics 10:783-796.

Chou H-H, Chiu H-C, Delaney NF, Segrè D, Marx CJ. 2011. Diminishing returns epistasis among beneficial mutations decelerates adaptation. Science 332:1190-1192.

Cingolani P, Platts A, Wang LL, Coon M, Nguyen T, Wang L, Land SJ, Lu X, Ruden DM. 2012. A program for annotating and predicting the effects of single nucleotide polymorphisms, SnpEff: SNPs in the genome of Drosophila melanogaster strain w1118; iso-2; iso-3. Fly 6:80-92.

Couce A, Tenaillon OA. 2015. The rule of declining adaptability in microbial evolution experiments. Frontiers in Genetics 6:1-6.

Echenique JIR, Rojas Echenique JI, Kryazhimskiy S, Nguyen Ba AN, Desai MM. 2019. Modular epistasis and the compensatory evolution of gene deletion mutants. PLOS Genetics 15:e1007958.

Fisher, Kaitlin J., Kryazhimskiy, Sergey, Lang, Gregory I. 2019. Detecting genetic interactions using parallel evolution in experimental populations. Philosophical Transactions of the Royal Society B: Biological Sciences 374:20180237.

Fisher KJ, Buskirk SW, Vignogna RC, Marad DA, Lang GI. 2018. Adaptive genome duplication affects patterns of molecular evolution in Saccharomyces cerevisiae. PLoS Genetics 14:e1007396.

Forche A, Abbey D, Pisithkul T, Weinzierl MA, Ringstrom T, Bruck D, Petersen K, Berman J. 2011. Stress alters rates and types of loss of heterozygosity in Candida albicans. MBio 2. doi:10.1128/mBio.00129-11

Frenkel EM, McDonald MJ, Van Dyken JD, Kosheleva K, Lang GI, Desai MM. 2015. Crowded growth leads to the spontaneous evolution of semistable coexistence in laboratory yeast populations. PNAS 112:1130611311.

Gaspar JM. 2018. NGmerge: merging paired-end reads via novel empirically-derived models of sequencing errors. BMC Bioinformatics 19:536.

Gerstein AC, Kuzmin A, Otto SP. 2014. Loss-of-heterozygosity facilitates passage through Haldane's sieve for Saccharomyces cerevisiae undergoing adaptation. Nature Communications 5:3819. 
Giaever G, Chu AM, Ni L, Connelly C, Riles L, Véronneau S, Dow S, Lucau-Danila A, Anderson K, André B, Arkin AP, Astromoff A, El-Bakkoury M, Bangham R, Benito R, Brachat S, Campanaro S, Curtiss M, Davis K, Deutschbauer A, Entian K-D, Flaherty P, Foury F, Garfinkel DJ, Gerstein M, Gotte D, Güldener U, Hegemann JH, Hempel S, Herman Z, Jaramillo DF, Kelly DE, Kelly SL, Kötter P, LaBonte D, Lamb DC, Lan N, Liang H, Liao H, Liu L, Luo C, Lussier M, Mao R, Menard P, Ooi SL, Revuelta JL, Roberts CJ, Rose M, Ross-Macdonald P, Scherens B, Schimmack G, Shafer B, Shoemaker DD, Sookhai-Mahadeo S, Storms RK, Strathern JN, Valle G, Voet M, Volckaert G, Wang C-Y, Ward TR, Wilhelmy J, Winzeler EA, Yang Y, Yen G, Youngman E, Yu K, Bussey H, Boeke JD, Snyder M, Philippsen P, Davis RW, Johnston M. 2002. Functional profiling of the Saccharomyces cerevisiae genome. Nature 418:387-391.

Good BH, Desai MM. 2016. Evolution of Mutation Rates in Rapidly Adapting Asexual Populations. Genetics 204:1249-1266.

Good BH, McDonald MJ, Barrick JE, Lenski RE, Desai MM. 2017. The dynamics of molecular evolution over 60,000 generations. Nature 551:45-50.

Harrison E, Koufopanou V, Burt A, MacLean RC. 2012. The cost of copy number in a selfish genetic element: the 2- $\mu \mathrm{m}$ plasmid of Saccharomyces cerevisiae. Journal of Evolutionary Biology 25:2348-2356.

Huang M-E, Rio A-G, Nicolas A, Kolodner RD. 2003. A genomewide screen in Saccharomyces cerevisiae for genes that suppress the accumulation of mutations. PNAS 100:11529-11534.

Jerison ER, Nguyen Ba AN, Desai MM, Kryazhimskiy S. 2020. Chance and necessity in the pleiotropic consequences of adaptation for budding yeast. Nature Ecology \& Evolution 4:601-611.

Johnson MS, Martsul A, Kryazhimskiy S, Desai MM. 2019. Higher-fitness yeast genotypes are less robust to deleterious mutations. Science 366:490-493.

Kassen R. 2014. Experimental evolution and the nature of biodiversity. Roberts.

Khan AI, Dinh DM, Schneider D, Lenski RE, Cooper TF. 2011. Negative epistasis between beneficial mutations in an evolving bacterial population. Science 332:1193-1196.

Klopfenstein DV, Zhang L, Pedersen BS, Ramírez F, Warwick Vesztrocy A, Naldi A, Mungall CJ, Yunes JM, Botvinnik O, Weigel M, Dampier W, Dessimoz C, Flick P, Tang H. 2018. GOATOOLS: A Python library for Gene Ontology analyses. Scientific Reports 8:10872.

Kokina A, Kibilds J, Liepins J. 2014. Adenine auxotrophy--be aware: some effects of adenine auxotrophy in Saccharomyces cerevisiae strain W303-1A. FEMS Yeast Research 14:697-707.

Kryazhimskiy S, Rice DP, Jerison ER, Desai MM. 2014. Global epistasis makes adaptation predictable despite sequence-level stochasticity. Science 344:1519-1522.

Lang GI, Botstein D, Desai MM. 2011. Genetic variation and the fate of beneficial mutations in asexual populations. Genetics 188:647-661.

Lang GI, Murray AW. 2008. Estimating the Per-Base-Pair Mutation Rate in the Yeast Saccharomyces cerevisiae. Genetics 178:67.

Lang GI, Rice DP, Hickman MJ, Sodergren E, Weinstock GM, Botstein D, Desai MM. 2013. Pervasive genetic hitchhiking and clonal interference in forty evolving yeast populations. Nature 500:571-574.

Layer RM, Chiang C, Quinlan AR, Hall IM. 2014. LUMPY: a probabilistic framework for structural variant discovery. Genome Biology 15:R84.

Leiby N, Marx CJ. 2014. Metabolic erosion primarily through mutation accumulation, and not tradeoffs, drives limited evolution of substrate specificity in Escherichia coli. PLoS Biology 12:e1001789.

Lenski RE. 2017. Experimental evolution and the dynamics of adaptation and genome evolution in microbial populations. The ISME Journal 11:2181-2194.

Leu J-Y, Chang S-L, Chao J-C, Woods LC, McDonald MJ. 2020. Sex alters molecular evolution in diploid experimental populations of S. cerevisiae. Nature Ecology \& Evolution 4:453-460. 
Li H. 2013. Aligning sequence reads, clone sequences and assembly contigs with BWA-MEM. arXiv, $1303.3997 \mathrm{v} 2$.

Li H, Handsaker B, Wysoker A, Fennell T, Ruan J, Homer N, Marth G, Abecasis G, Durbin R, 1000 Genome Project Data Processing Subgroup. 2009. The Sequence Alignment/Map format and SAMtools. Bioinformatics 25:2078-2079.

Liu G, Yong MYJ, Yurieva M, Srinivasan KG, Liu J, Lim JSY, Poidinger M, Wright GD, Zolezzi F, Choi H, Pavelka N, Rancati G. 2015. Gene Essentiality Is a Quantitative Property Linked to Cellular Evolvability. Cell 163:1388-1399.

Marad DA, Buskirk SW, Lang GI. 2018. Altered access to beneficial mutations slows adaptation and biases fixed mutations in diploids. Nature Ecology \& Evolution 2:882-889.

McDonald MJ, Rice DP, Desai MM. 2016. Sex speeds adaptation by altering the dynamics of molecular evolution. Nature 531:233-236.

McKenna A, Hanna M, Banks E, Sivachenko A, Cibulskis K, Kernytsky A, Garimella K, Altshuler D, Gabriel S, Daly M, DePristo MA. 2010. The Genome Analysis Toolkit: a MapReduce framework for analyzing nextgeneration DNA sequencing data. Genome Research 20:1297-1303.

Murray AW. 2020. Can gene-inactivating mutations lead to evolutionary novelty? Current Biology 30:R465R471.

Oberacker P, Stepper P, Bond DM, Höhn S, Focken J, Meyer V, Schelle L, Sugrue VJ, Jeunen G-J, Moser T, Hore SR, von Meyenn F, Hipp K, Hore TA, Jurkowski TP. 2019. Bio-On-Magnetic-Beads (BOMB): Open platform for high-throughput nucleic acid extraction and manipulation. PLoS Biology 17:e3000107.

Ostrowski EA, Rozen DE, Lenski RE. 2005. Pleiotropic effects of beneficial mutations in Escherichia coli. Evolution 59:2343-2352.

Plucain J, Hindré T, Le Gac M, Tenaillon O, Cruveiller S, Médigue C, Leiby N, Harcombe WR, Marx CJ, Lenski $\mathrm{RE}$, Schneider D. 2014. Epistasis and allele specificity in the emergence of a stable polymorphism in Escherichia coli. Science 343:1366-1369.

Radchenko EA, McGinty RJ, Aksenova AY, Neil AJ, Mirkin SM. 2018. Quantitative Analysis of the Rates for Repeat-Mediated Genome Instability in a Yeast Experimental System In: Muzi-Falconi M, Brown GW, editors. Genome Instability: Methods and Protocols. New York, NY: Springer New York. pp. 421-438.

Roman H. 1956. Studies of gene mutation in Saccharomyces. Cold Spring Harbor Symposia on Quantitative Biology 21:175-185.

Rozen DE, Lenski RE. 2000. Long-Term Experimental Evolution in Escherichia coli. VIII. Dynamics of a Balanced Polymorphism. The American Naturalist 155:24-35.

Sarkar S, Ma WT, Sandri GH. 1992. On fluctuation analysis: a new, simple and efficient method for computing the expected number of mutants. Genetica 85:173-179.

Schmitt MJ, Breinig F. 2002. The viral killer system in yeast: from molecular biology to application. FEMS Microbiology Reviews 26:257-276.

Sharma KG, Kaur R, Bachhawat AK. 2003. The glutathione-mediated detoxification pathway in yeast: an analysis using the red pigment that accumulates in certain adenine biosynthetic mutants of yeasts reveals the involvement of novel genes. Archives of Microbiology 180:108-117.

Sniegowski PD, Gerrish PJ, Lenski RE. 1997. Evolution of high mutation rates in experimental populations of E. coli. Nature 387:703-705.

St Charles J, Hazkani-Covo E, Yin Y, Andersen SL, Dietrich FS, Greenwell PW, Malc E, Mieczkowski P, Petes TD. 2012. High-resolution genome-wide analysis of irradiated (UV and $\gamma$-rays) diploid yeast cells reveals a high frequency of genomic loss of heterozygosity (LOH) events. Genetics 190:1267-1284. 
Storici F, Resnick MA. 2006. The delitto perfetto approach to in vivo site-directed mutagenesis and chromosome rearrangements with synthetic oligonucleotides in yeast. Methods in Enzymology 409:329345.

Tenaillon O, Barrick JE, Ribeck N, Deatherage DE, Blanchard JL, Dasgupta A, Wu GC, Wielgoss S, Cruveiller S, Médigue C, Schneider D, Lenski RE. 2016. Tempo and mode of genome evolution in a 50,000-generation experiment. Nature 536:165-170.

Tenaillon O, Rodríguez-Verdugo A, Gaut RL, McDonald P, Bennett AF, Long AD, Gaut BS. 2012. The molecular diversity of adaptive convergence. Science 335:457-461.

Venkataram S, Dunn B, Li Y, Agarwala A, Chang J, Ebel ER, Geiler-Samerotte K, Hérissant L, Blundell JR, Levy SF, Fisher DS, Sherlock G, Petrov DA. 2016. Development of a Comprehensive Genotype-to-Fitness Map of Adaptation-Driving Mutations in Yeast. Cell 166:1585-1596.e22.

Walker BJ, Abeel T, Shea T, Priest M, Abouelliel A, Sakthikumar S, Cuomo CA, Zeng Q, Wortman J, Young SK, Earl AM. 2014. Pilon: an integrated tool for comprehensive microbial variant detection and genome assembly improvement. PLoS One 9:e112963.

Weinstein LA, Capaldo-Kimball F, Leibowitz MJ. 1993. Genetics of heat-curability of killer virus of yeast. Yeast 9:411-418.

Wielgoss S, Barrick JE, Tenaillon O, Wiser MJ, Dittmar WJ, Cruveiller S, Chane-Woon-Ming B, Médigue C, Lenski RE, Schneider D. 2013. Mutation rate dynamics in a bacterial population reflect tension between adaptation and genetic load. PNAS 110:222-227.

Wiser MJ, Ribeck N, Lenski RE. 2013. Long-term dynamics of adaptation in asexual populations. Science 342:1364-1367.

Woods DR, Bevan EA. 1968. Studies on the nature of the killer factor produced by Saccharomyces cerevisiae. Journal of General Microbiology 51:115-126.

Zeyl C, Vanderford T, Carter M. 2003. An Evolutionary Advantage of Haploidy in Large Yeast Populations. Science. 299:555-558. 


\section{Figure Supplements}

\begin{tabular}{|l|l|}
\hline Environment & \multicolumn{1}{|c|}{ Populations lost during evolution due to evaporation, pipetting errors, or contamination } \\
\hline & $\begin{array}{l}\text { P1A02 (blank);P1A11 (blank);P1D07 (blank);P1D08 (blank);P1E06 (blank);P1E07 (blank);P1E08 } \\
\text { (blank);P1G03 (blank);P1H03 (blank);P1B08 (contaminated);P1G02 (contaminated);P1G06 } \\
\text { (contaminated);P1B09 (contaminated);P1D02 (contaminated);P1F12 (contaminated);P1H01 } \\
\text { (contaminated);P1A07 (contaminated);P1D11 (contaminated) }\end{array}$ \\
\hline YPD 30 $\mathrm{C}$ & $\begin{array}{l}\text { P2A08 (blank);P2D07 (blank);P2E01 (blank);P2E05 (blank);P2E07 (blank);P2F10 (blank);P2G03 } \\
\text { (blank);P2G07 (blank); }\end{array}$ \\
\hline & $\begin{array}{l}\text { P3A01 (blank);P3A02 (blank);P3A04 (blank);P3A05 (blank);P3A06 (blank);P3A07 (blank);P3A08 } \\
\text { (blank);P3A09 (blank);P3A11 (blank);P3B01 (blank);P3B02 (blank);P3B03 (blank);P3B04 } \\
\text { (blank);P3B05 (blank);P3B09 (blank);P3B12 (blank);P3C01 (blank);P3C02 (blank);P3C08 } \\
\text { (blank);P3C12 (blank);P3D01 (blank);P3D07 (blank);P3D08 (blank);P3D12 (blank);P3E01 } \\
\text { (blank);P3E05 (blank);P3E06 (blank);P3E07 (blank);P3E09 (blank);P3F01 (blank);P3G01 } \\
\text { (blank);P3H01 (blank);P3H06 (blank);P3H07 (blank);P3H08 (blank);P3H09 (blank);P3H10 } \\
\text { (blank);P3H11 (blank);P3H12 (blank) }\end{array}$ \\
\hline SC 37C &
\end{tabular}

Figure 1 - figure supplement 1. Record of populations lost during evolution. 
A
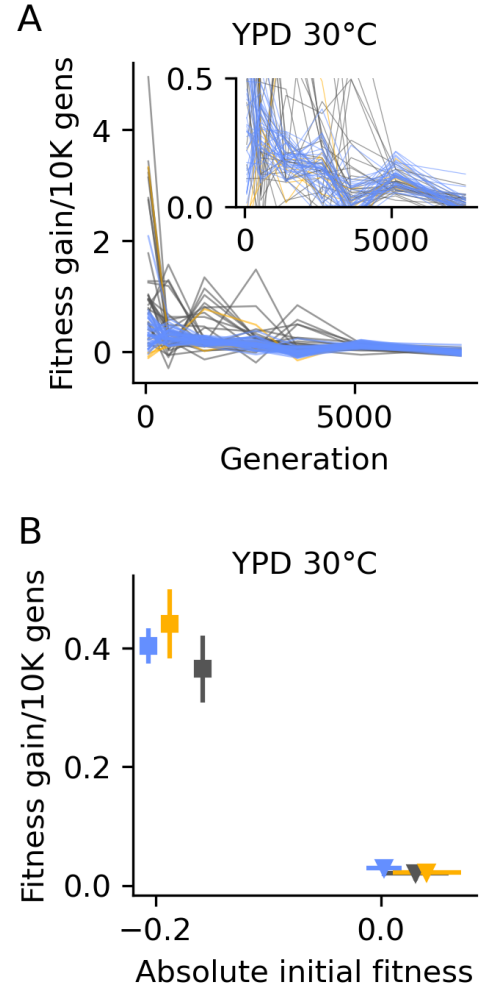
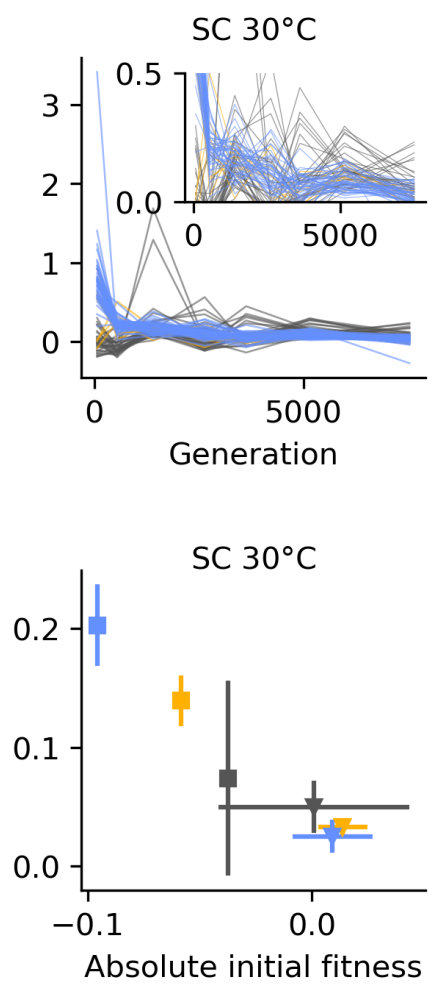

$\mathrm{SC} 37^{\circ} \mathrm{C}$

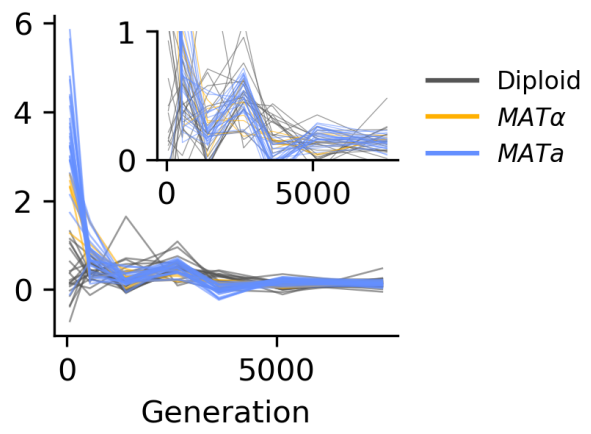

SC $37^{\circ} \mathrm{C}$

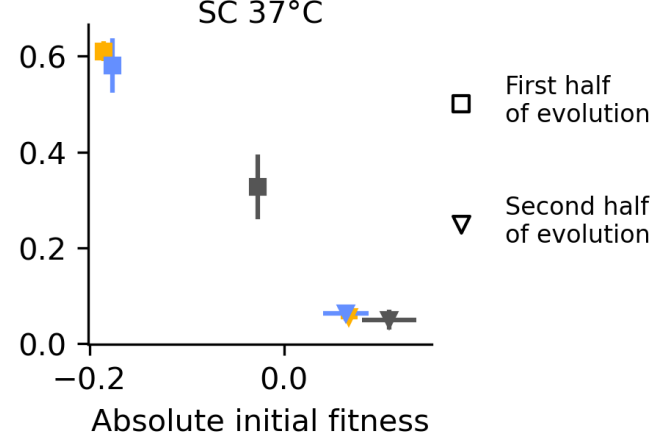

Figure 2 - figure supplement 1. Declining adaptability. (A) Fitness increase rate (per 10,000 generations) between timepoints over the course of evolution. The insets are the same data, but with a cut-off y-axis. (B) For each strain and environment, the mean rate of fitness gain over the first half of evolution (square points) and the second half of evolution (triangle points), as a function of initial fitness or the mean fitness at the midpoint, respectively. Colors are the same as in (A). Error bars represent standard deviations. 

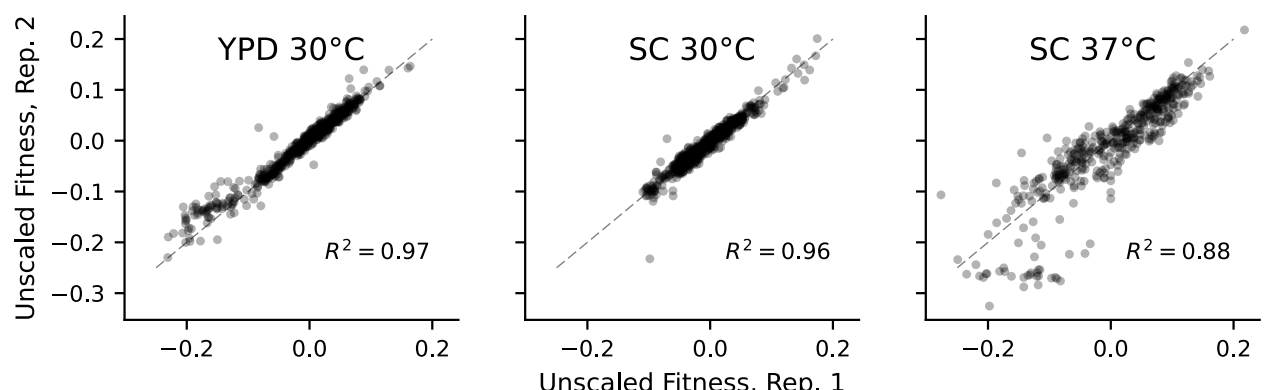

Figure 2 - figure supplement 2. Correlations between absolute fitness measured in replicate competitions with a fluorescent reference. 


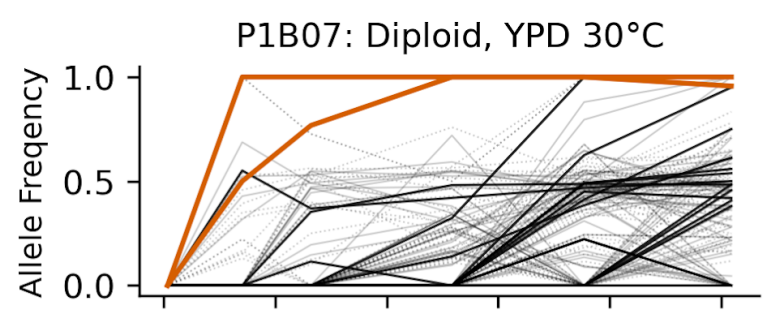

P1C08: Diploid, YPD $30^{\circ} \mathrm{C}$

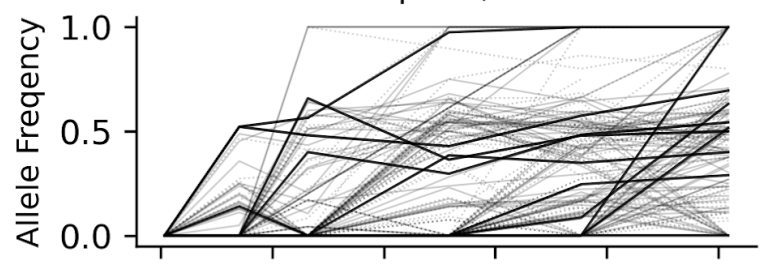

P1D09: Diploid, YPD $30^{\circ} \mathrm{C}$

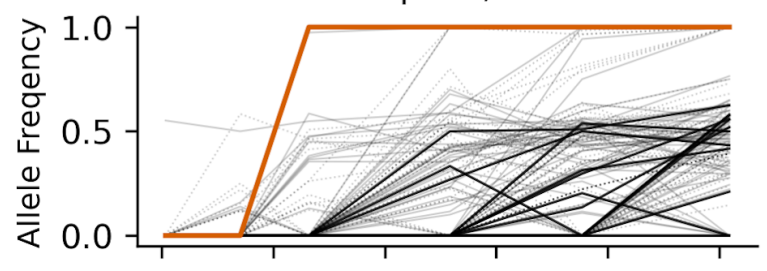

P1F07: Diploid, YPD $30^{\circ} \mathrm{C}$

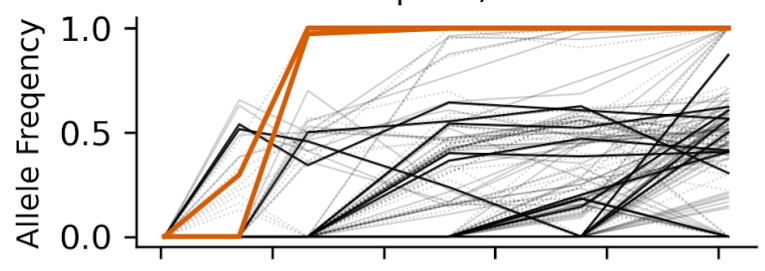

P1F10: Diploid, YPD $30^{\circ} \mathrm{C}$

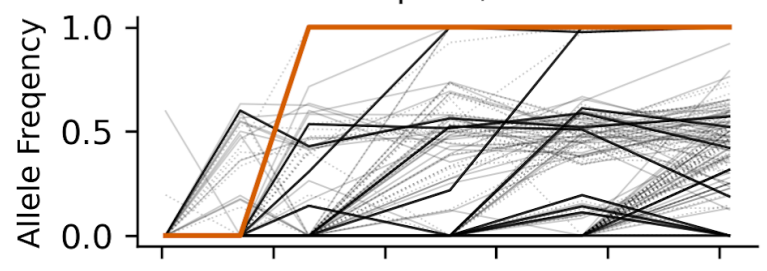

P1G09: Diploid, YPD $30^{\circ} \mathrm{C}$

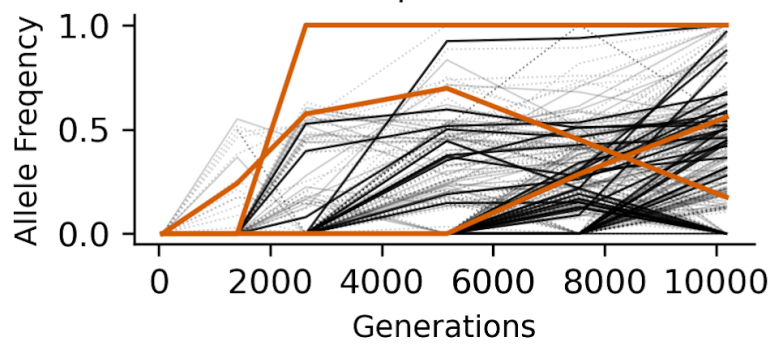

P1C07: Diploid, YPD $30^{\circ} \mathrm{C}$

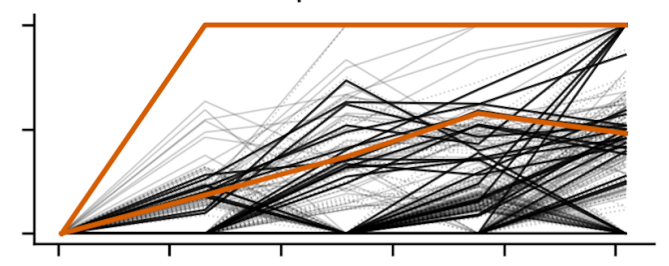

P1C09: Diploid, YPD $30^{\circ} \mathrm{C}$

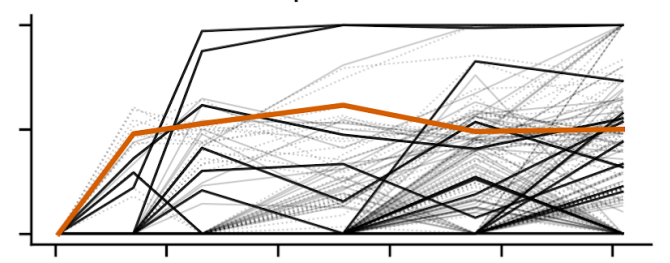

P1E09: Diploid, YPD $30^{\circ} \mathrm{C}$

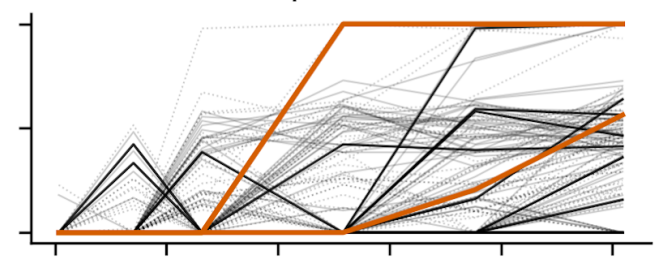

P1F08: Diploid, YPD $30^{\circ} \mathrm{C}$

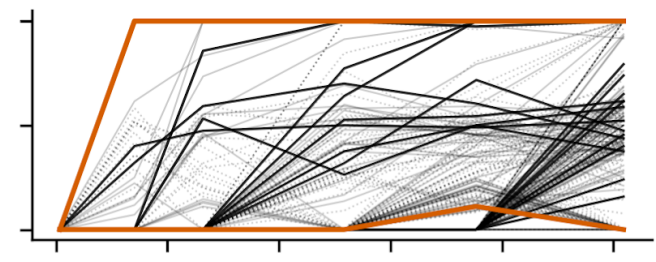

P1G08: Diploid, YPD $30^{\circ} \mathrm{C}$

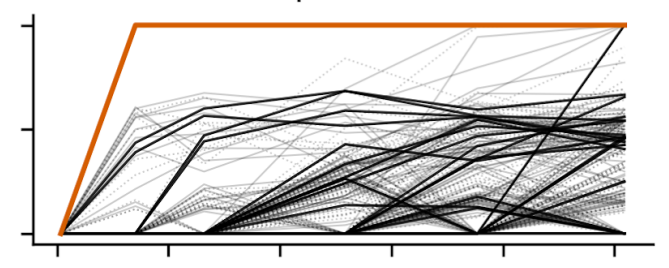

P1G10: Diploid, YPD $30^{\circ} \mathrm{C}$

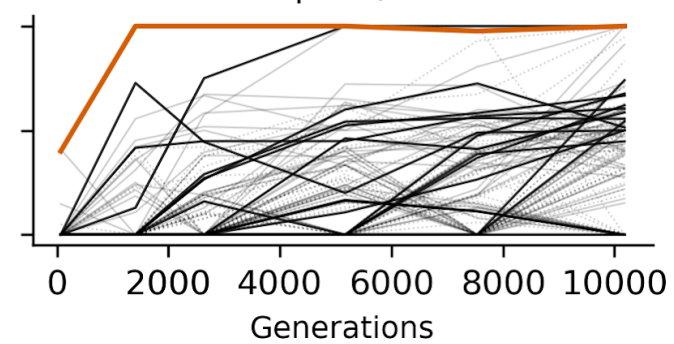

Figure 3 - figure supplement 1. Allele frequencies over time in all focal diploid populations in YPD $30^{\circ} \mathrm{C}$. Nonsynonymous mutations in "multi-hit" genes are solid black lines, mutations in the adenine biosynthesis pathway are colored orange, other nonsynonymous mutations are thin grey lines, and synonymous mutations are dotted lines. 

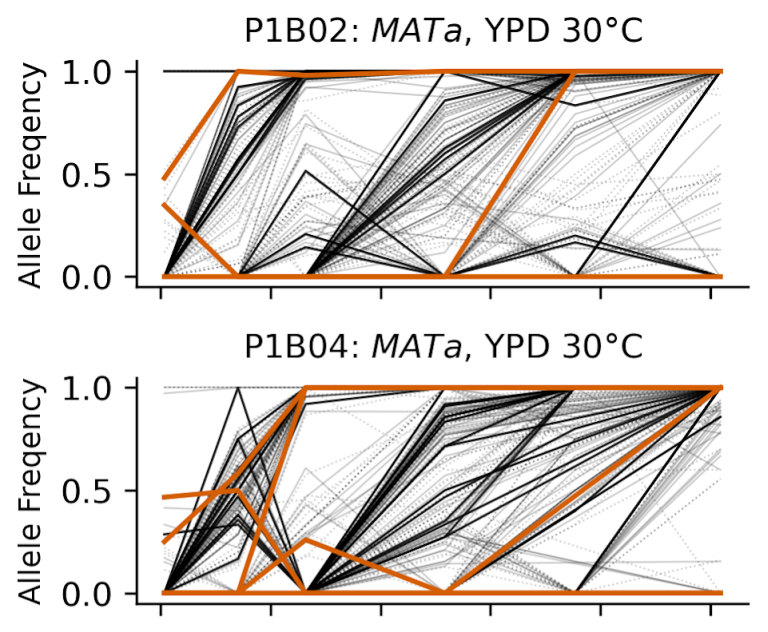

P1C04: MATa, YPD $30^{\circ} \mathrm{C}$

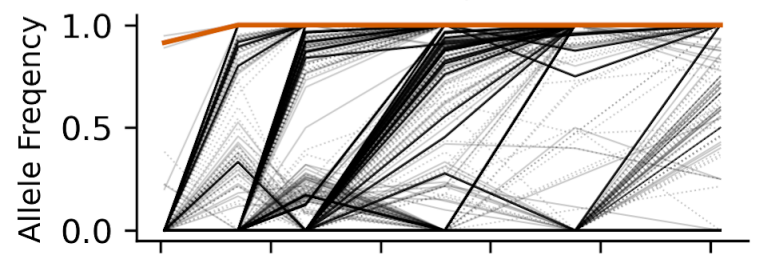

P1C06: MATa, YPD $30^{\circ} \mathrm{C}$

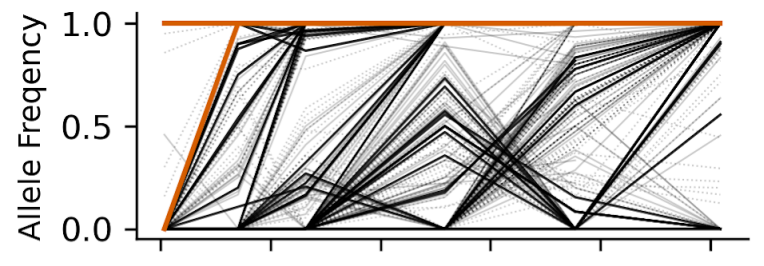

P1E04: MATa, YPD $30^{\circ} \mathrm{C}$

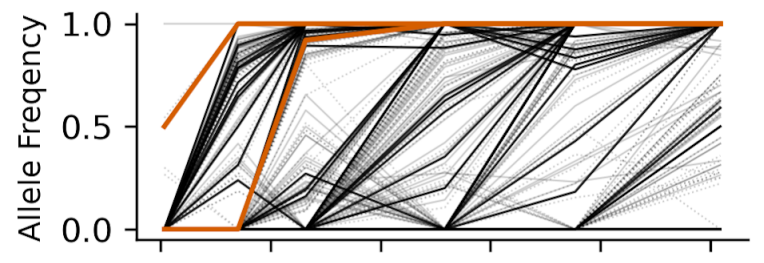

P1G04: MATa, YPD $30^{\circ} \mathrm{C}$

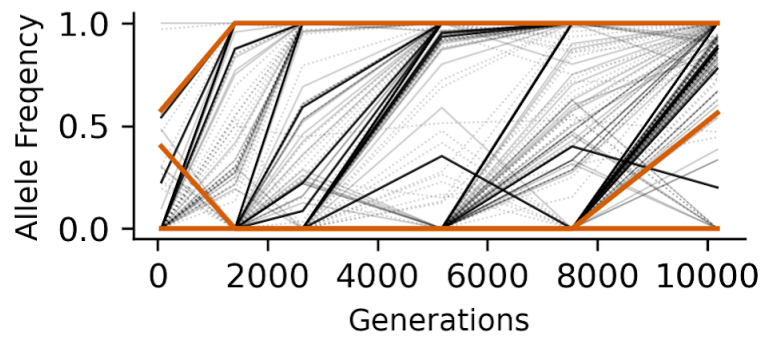

P1B03: MATa, YPD $30^{\circ} \mathrm{C}$

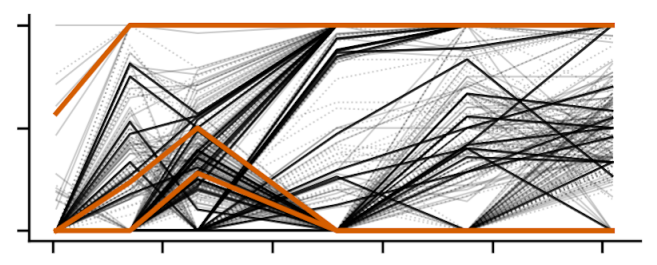

P1C02: MATa, YPD $30^{\circ} \mathrm{C}$

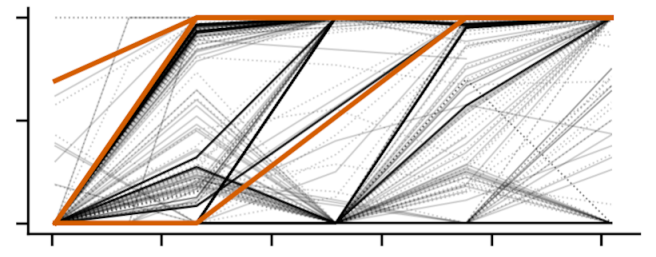

P1C05: MATa, YPD $30^{\circ} \mathrm{C}$

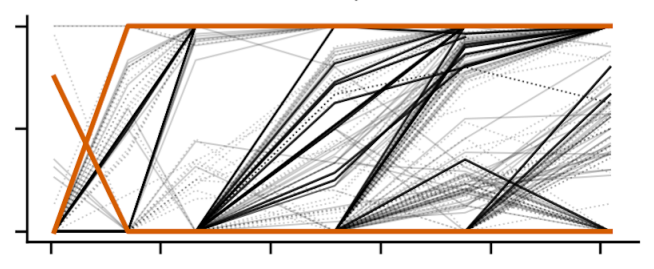

P1D03: MATa, YPD $30^{\circ} \mathrm{C}$

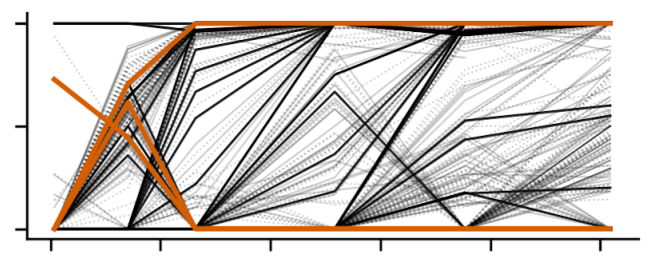

P1F05: MATa, YPD $30^{\circ} \mathrm{C}$

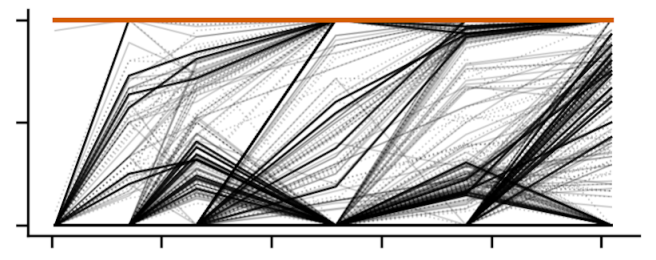

P1G05: MATa, YPD $30^{\circ} \mathrm{C}$

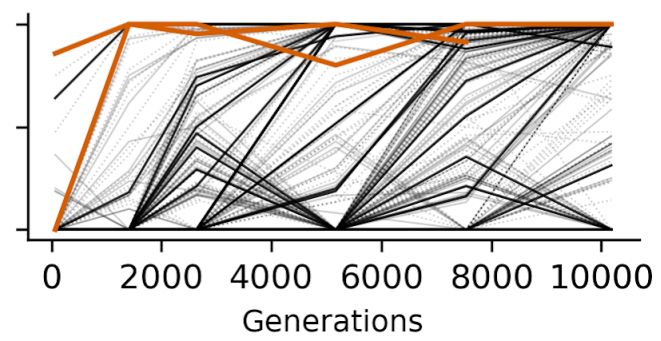

Figure 3 - figure supplement 2. Allele frequencies over time in all focal MATa populations in YPD $30^{\circ} \mathrm{C}$. Nonsynonymous mutations in "multi-hit" genes are solid black lines, mutations in the adenine biosynthesis pathway are colored orange, other nonsynonymous mutations are thin grey lines, and synonymous mutations are dotted lines. 


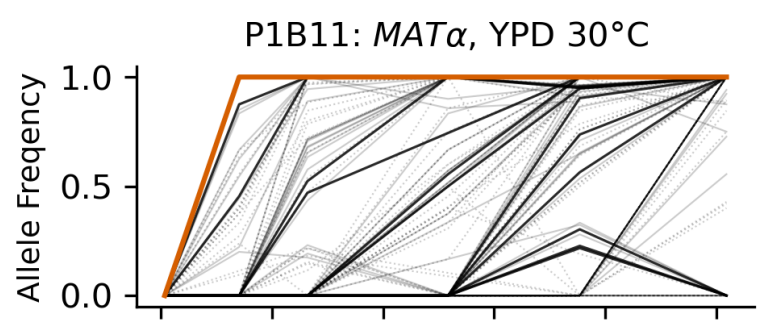

P1E11: MAT $\alpha$, YPD $30^{\circ} \mathrm{C}$

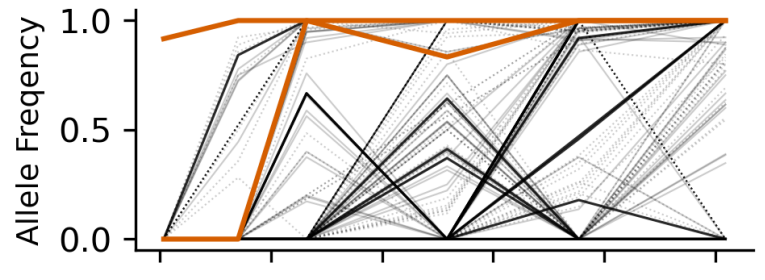

P1G11: MAT $\alpha$, YPD $30^{\circ} \mathrm{C}$

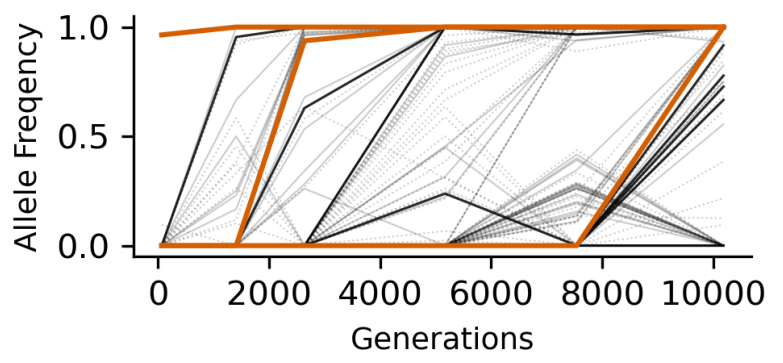

P1C11: MAT $\alpha$, YPD $30^{\circ} \mathrm{C}$

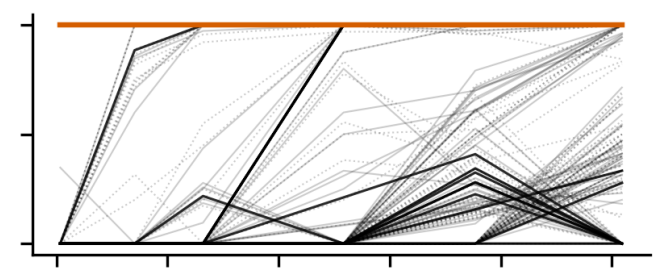

P1F11: MAT $\alpha$, YPD $30^{\circ} \mathrm{C}$

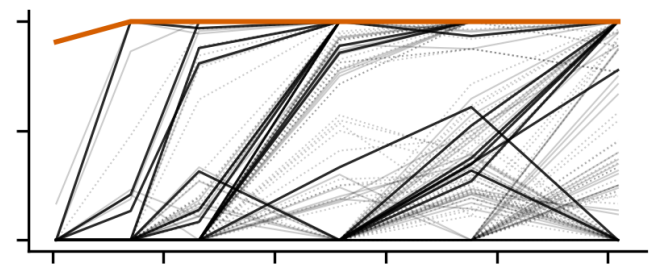

P1H11: MAT $\alpha$, YPD $30^{\circ} \mathrm{C}$

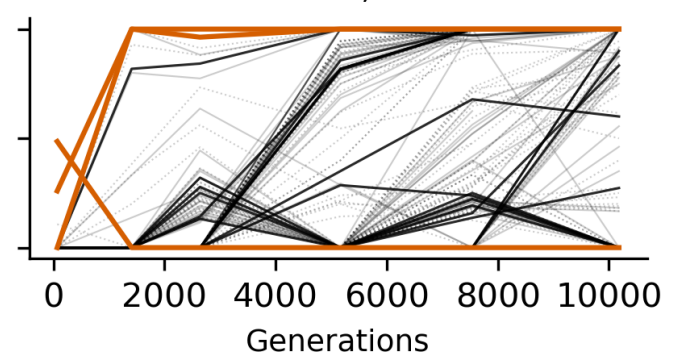

Figure 3 - figure supplement 3. Allele frequencies over time in all focal MAT $\alpha$ populations in YPD $30^{\circ} \mathrm{C}$. Nonsynonymous mutations in "multi-hit" genes are solid black lines, mutations in the adenine biosynthesis pathway are colored orange, other nonsynonymous mutations are thin grey lines, and synonymous mutations are dotted lines. 


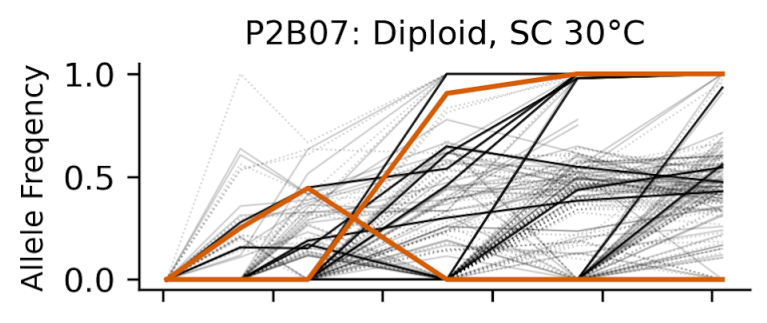

P2B09: Diploid, SC $30^{\circ} \mathrm{C}$

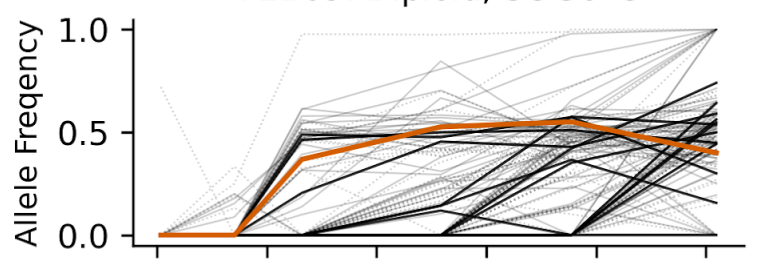

P2C10: Diploid, $\mathrm{SC} 30^{\circ} \mathrm{C}$

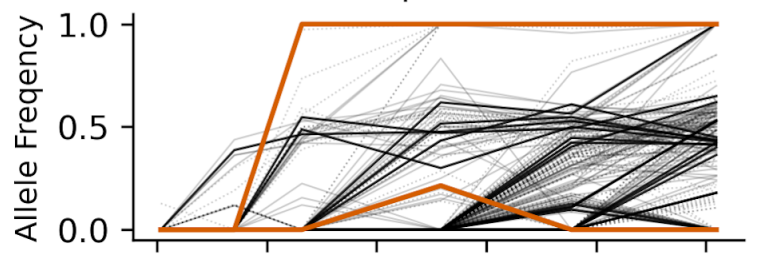

P2E08: Diploid, SC $30^{\circ} \mathrm{C}$

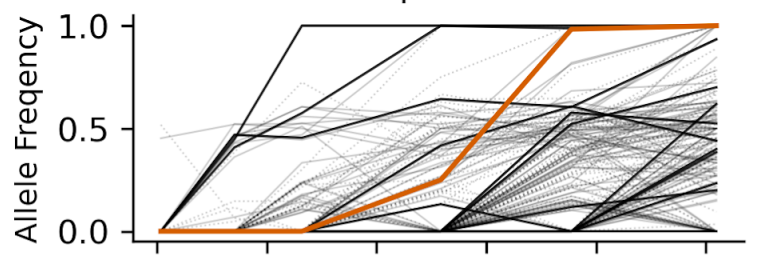

P2F09: Diploid, $\mathrm{SC} 30^{\circ} \mathrm{C}$

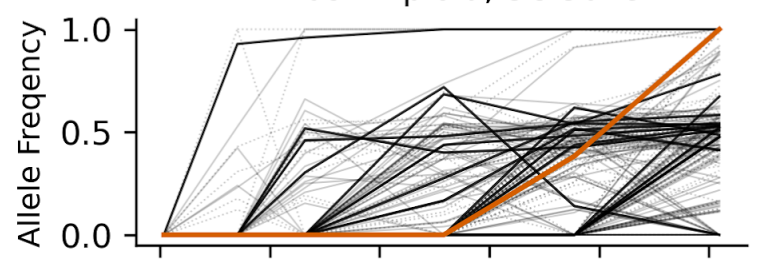

P2G09: Diploid, $\mathrm{SC} 30^{\circ} \mathrm{C}$

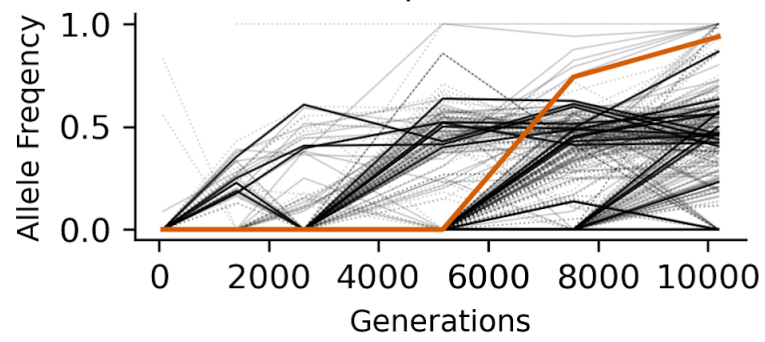

P2B08: Diploid, $\mathrm{SC} 30^{\circ} \mathrm{C}$

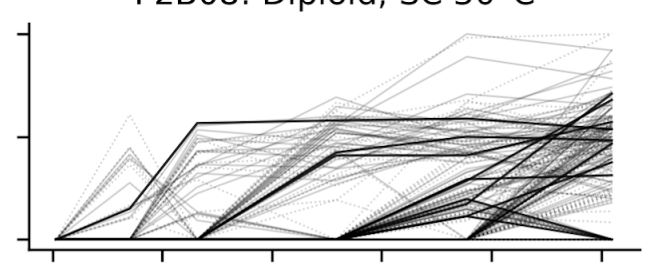

P2B10: Diploid, SC $30^{\circ} \mathrm{C}$

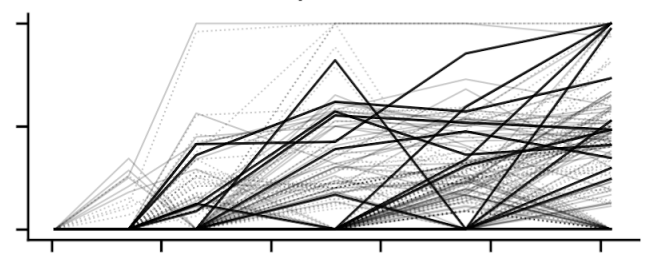

P2D08: Diploid, SC $30^{\circ} \mathrm{C}$

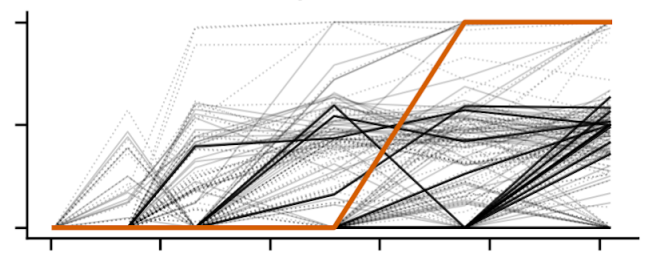

P2F07: Diploid, SC $30^{\circ} \mathrm{C}$

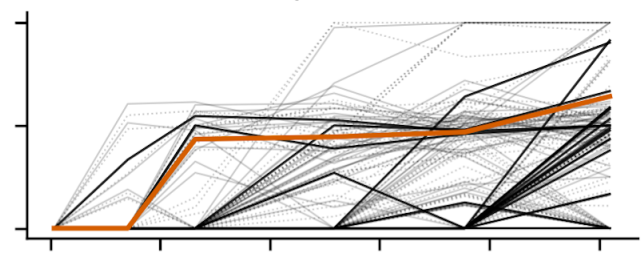

P2G08: Diploid, SC $30^{\circ} \mathrm{C}$

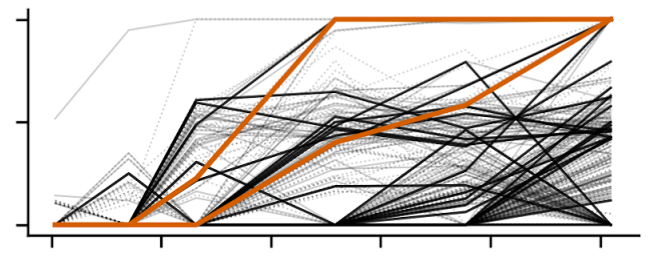

P2G10: Diploid, SC $30^{\circ} \mathrm{C}$

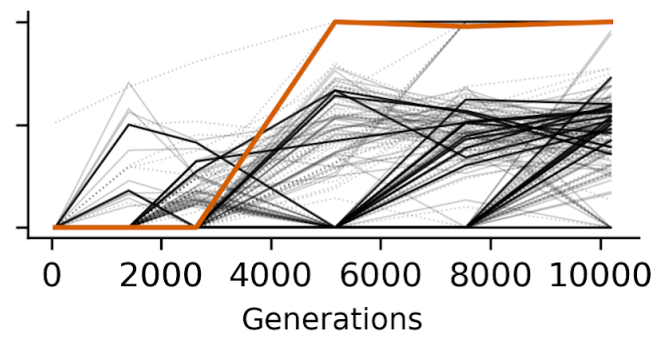

Figure 3 - figure supplement 4. Allele frequencies over time in all focal diploid populations in SC $30^{\circ} \mathrm{C}$. Nonsynonymous mutations in "multi-hit" genes are solid black lines, mutations in the adenine biosynthesis pathway are colored orange, other nonsynonymous mutations are thin grey lines, and synonymous mutations are dotted lines. 
P2B04: $\mathrm{MATa}, \mathrm{SC} 30^{\circ} \mathrm{C}$

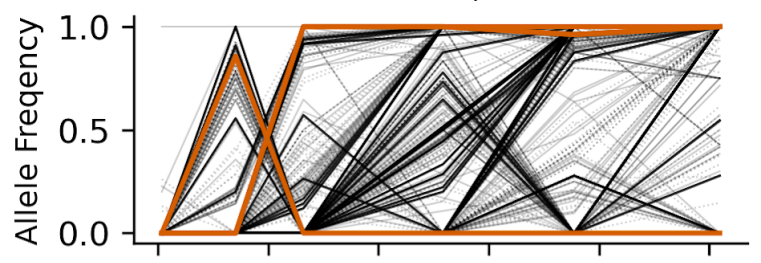

P2C02: $\mathrm{MATa}, \mathrm{SC} 30^{\circ} \mathrm{C}$

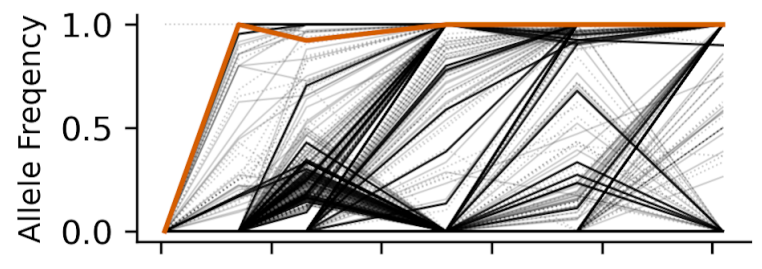

P2C05: MATa, SC $30^{\circ} \mathrm{C}$

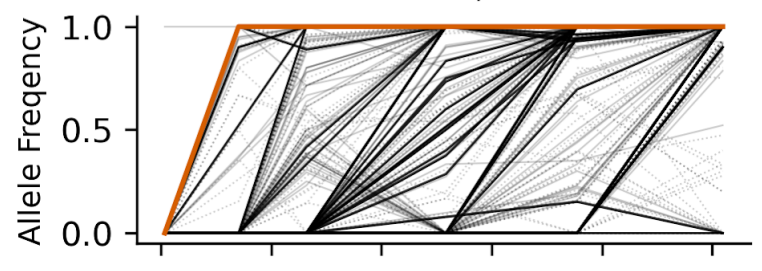

P2D03: $\mathrm{MATa}, \mathrm{SC} 30^{\circ} \mathrm{C}$

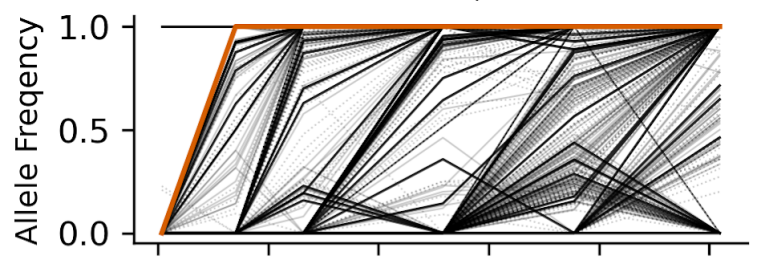

P2E06: $\mathrm{MATa}, \mathrm{SC} 30^{\circ} \mathrm{C}$

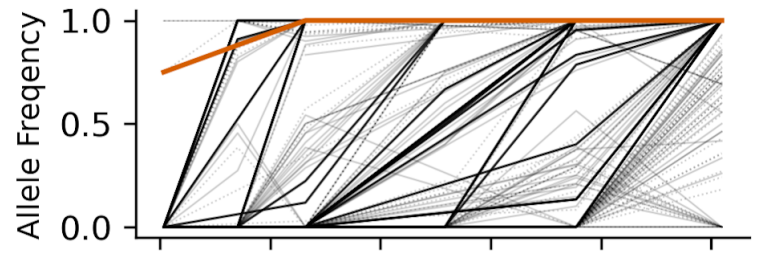

P2G04: MATa, SC $30^{\circ} \mathrm{C}$

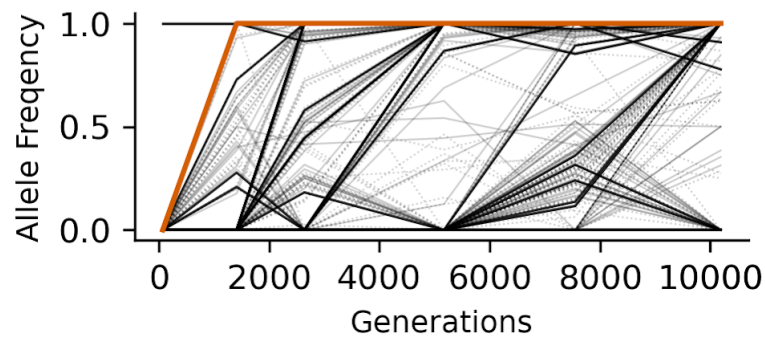

P2B05: MATa, $\mathrm{SC} 30^{\circ} \mathrm{C}$

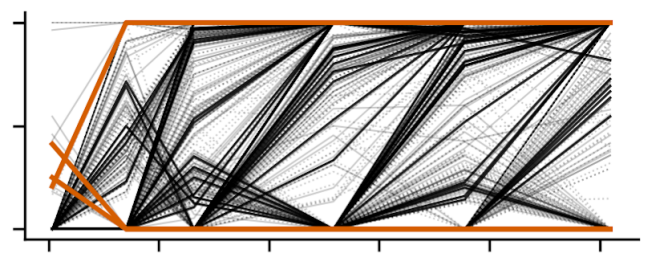

P2C04: MATa, SC $30^{\circ} \mathrm{C}$

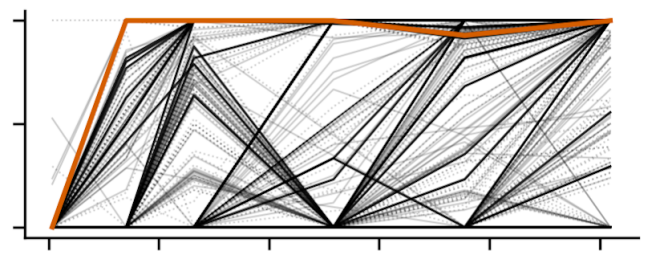

P2C06: $\mathrm{MATa}, \mathrm{SC} 30^{\circ} \mathrm{C}$

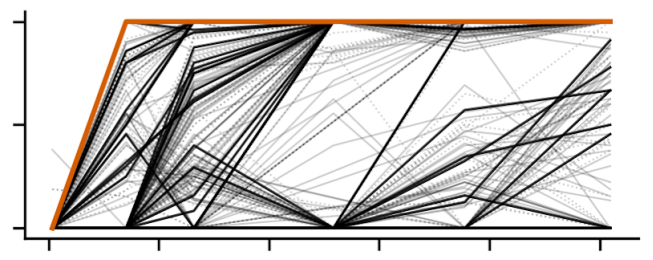

P2D06: MATa, SC $30^{\circ} \mathrm{C}$

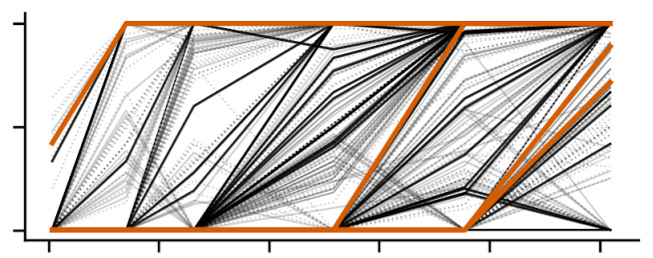

P2F02: MATa, $\mathrm{SC} 30^{\circ} \mathrm{C}$

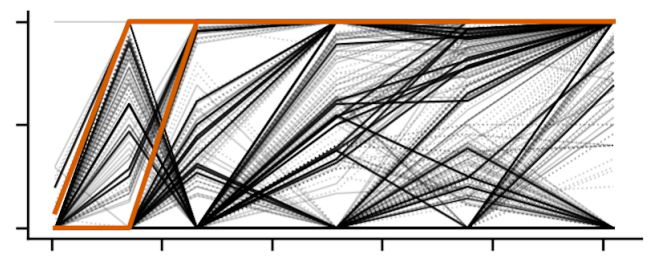

P2G05: MATa, SC $30^{\circ} \mathrm{C}$

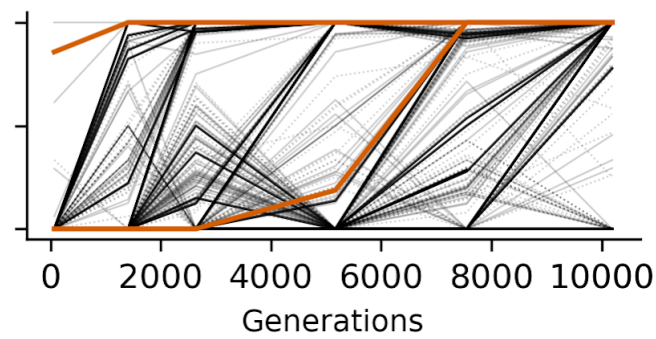

Figure 3 - figure supplement 5. Allele frequencies over time in all focal MATa populations in SC $30^{\circ} \mathrm{C}$. Nonsynonymous mutations in "multi-hit" genes are solid black lines, mutations in the adenine biosynthesis pathway are colored orange, other nonsynonymous mutations are thin grey lines, and synonymous mutations are dotted lines. 


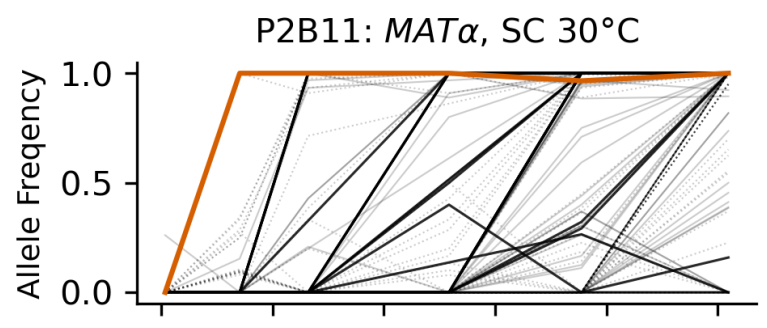

P2D11: $M A T \alpha, S C 30^{\circ} \mathrm{C}$

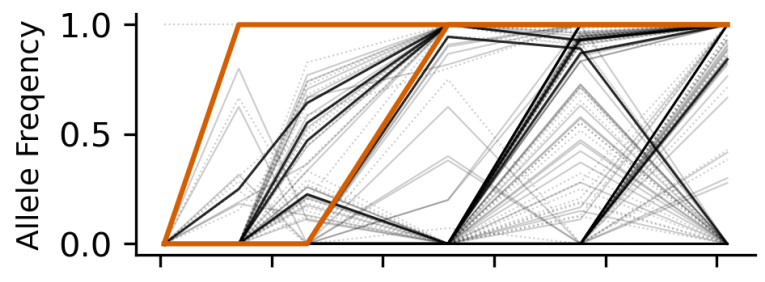

P2F11: $M A T \alpha, S C 30^{\circ} \mathrm{C}$

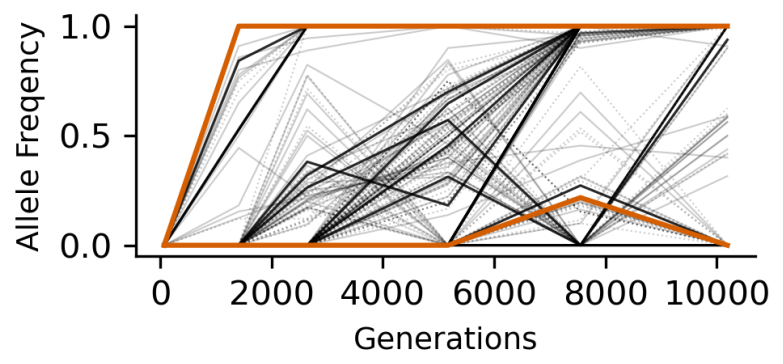

P2C11: $\mathrm{MAT} \alpha, \mathrm{SC} 30^{\circ} \mathrm{C}$

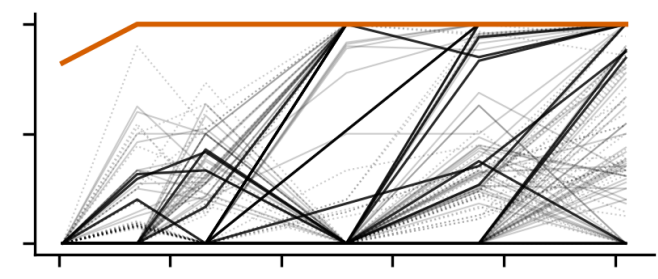

P2E11: $M A T \alpha, S C 30^{\circ} \mathrm{C}$

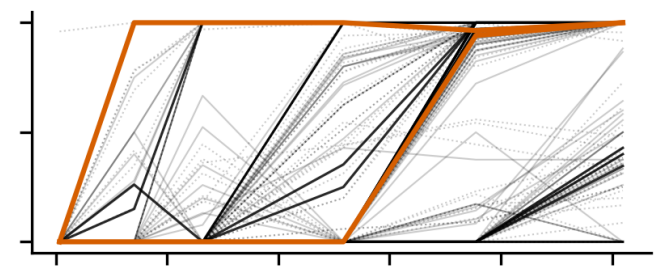

P2G11: $M A T \alpha, S C 30^{\circ} \mathrm{C}$

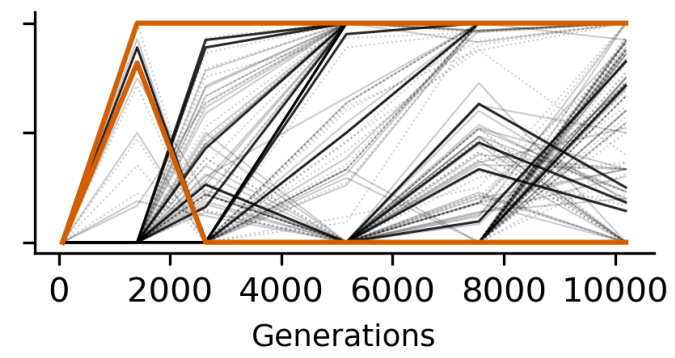

Figure 3 - figure supplement 6. Allele frequencies over time in all focal MAT $\alpha$ populations in SC $30^{\circ} \mathrm{C}$. Nonsynonymous mutations in "multi-hit" genes are solid black lines, mutations in the adenine biosynthesis pathway are colored orange, other nonsynonymous mutations are thin grey lines, and synonymous mutations are dotted lines. 
bioRxiv preprint doi: https:/doi.org/10.1101/202010.09330191. this version posted October 10,2020. The copyright holder for this preprint (which was not certified by peer review) is the author/funder, who has granted bioRxiv a license to display the preprint in perpetuity. It is made available under aCC-BY-ND 4.0 International license.

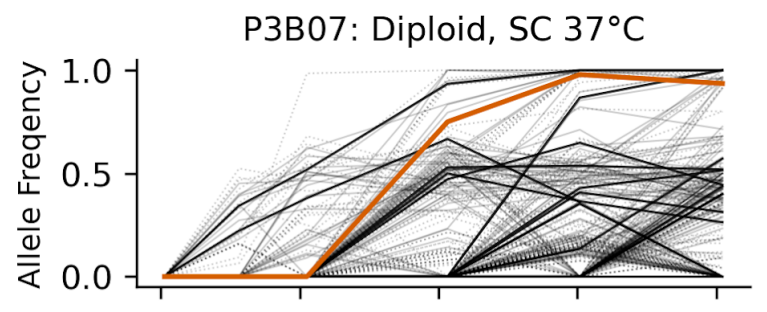

P3B10: Diploid, SC $37^{\circ} \mathrm{C}$

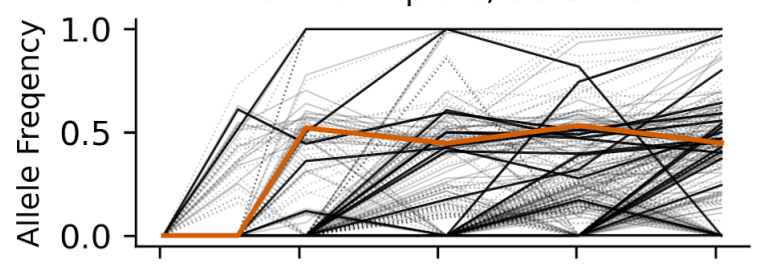

P3C10: Diploid, SC $37^{\circ} \mathrm{C}$

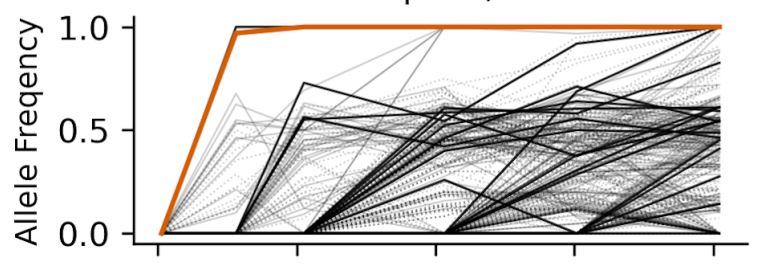

P3D10: Diploid, SC $37^{\circ} \mathrm{C}$

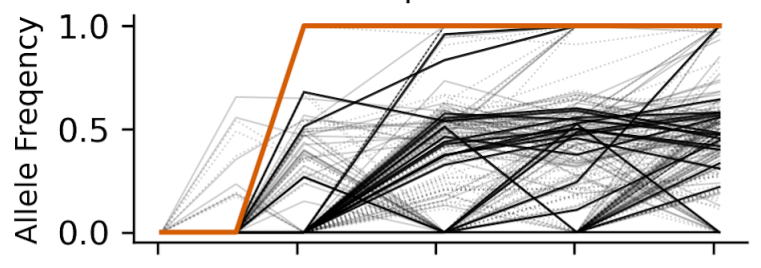

P3F07: Diploid, SC $37^{\circ} \mathrm{C}$

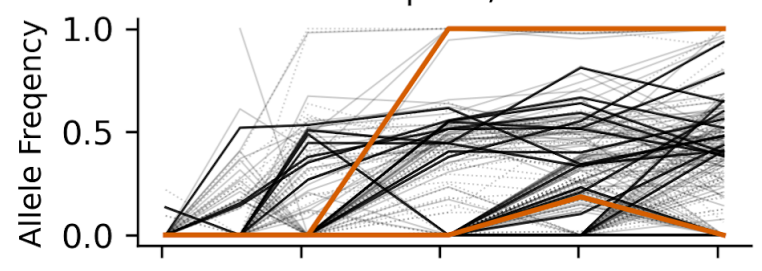

P3G09: Diploid, SC $37^{\circ} \mathrm{C}$

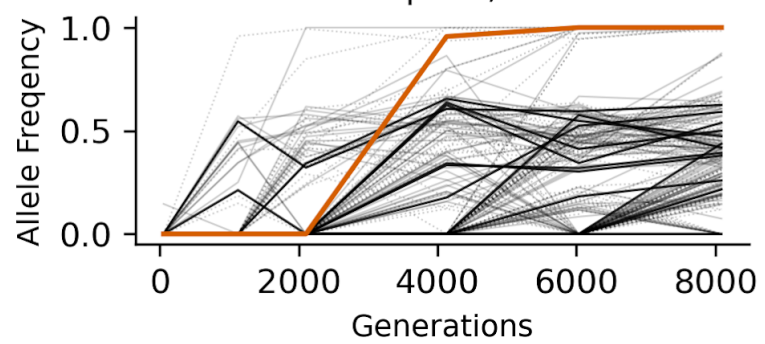

P3B08: Diploid, SC $37^{\circ} \mathrm{C}$

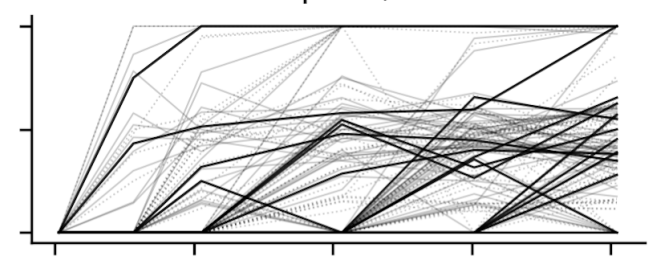

P3C07: Diploid, SC $37^{\circ} \mathrm{C}$

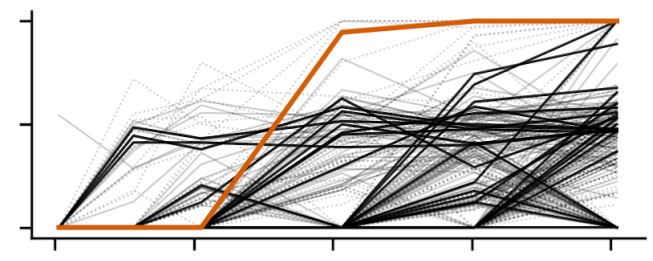

P3D09: Diploid, SC $37^{\circ} \mathrm{C}$

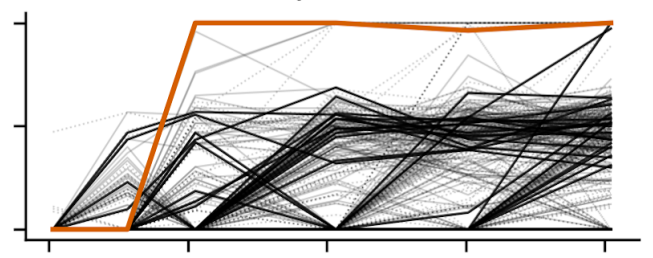

P3E08: Diploid, SC $37^{\circ} \mathrm{C}$

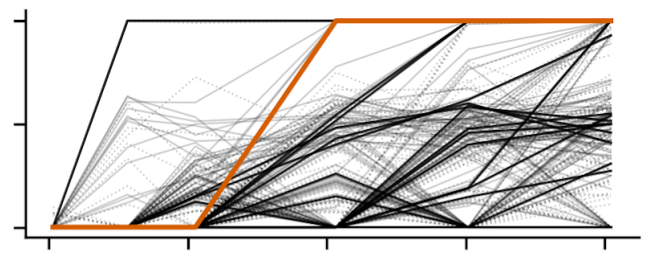

P3F09: Diploid, $\mathrm{SC} 37^{\circ} \mathrm{C}$

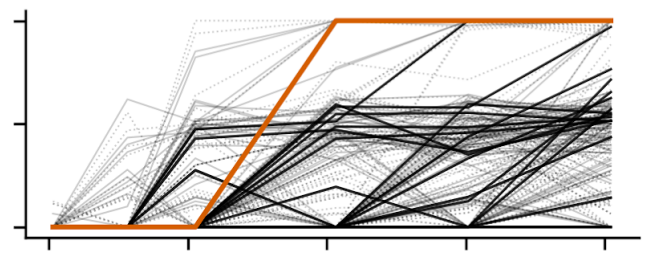

P3G10: Diploid, SC $37^{\circ} \mathrm{C}$

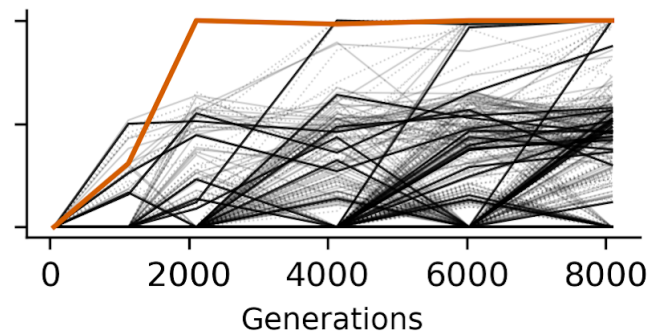

Figure 3 - figure supplement 7. Allele frequencies over time in all focal diploid populations in SC $37^{\circ} \mathrm{C}$. Nonsynonymous mutations in "multi-hit" genes are solid black lines, mutations in the adenine biosynthesis pathway are colored orange, other nonsynonymous mutations are thin grey lines, and synonymous mutations are dotted lines. 

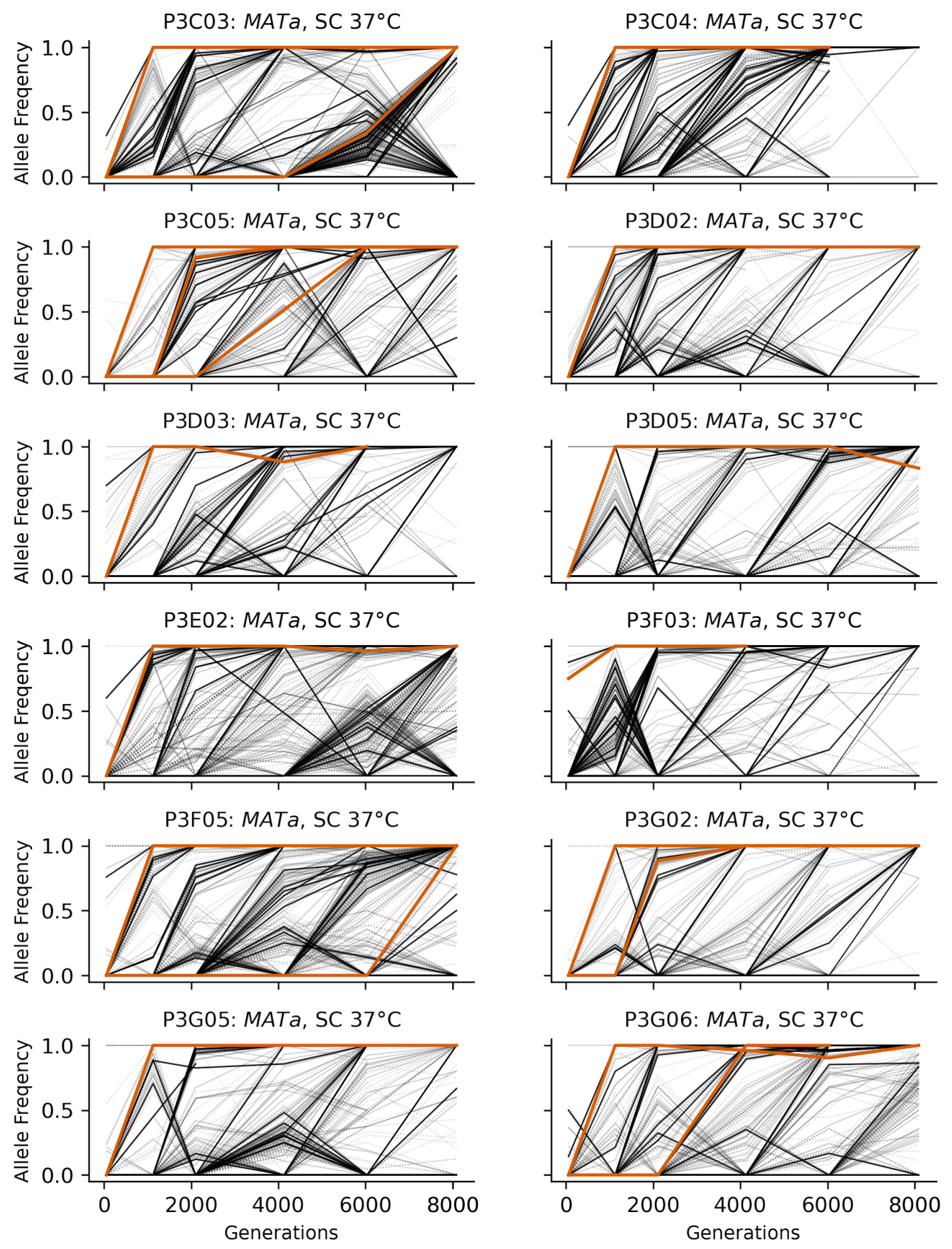

Figure 3 - figure supplement 8. Allele frequencies over time in all focal MATa populations in SC $37^{\circ} \mathrm{C}$. Nonsynonymous mutations in "multi-hit" genes are solid black lines, mutations in the adenine biosynthesis pathway are colored orange, other nonsynonymous mutations are thin grey lines, and synonymous mutations are dotted lines. 
P3B11: MAT $\alpha, \mathrm{SC} 37^{\circ} \mathrm{C}$

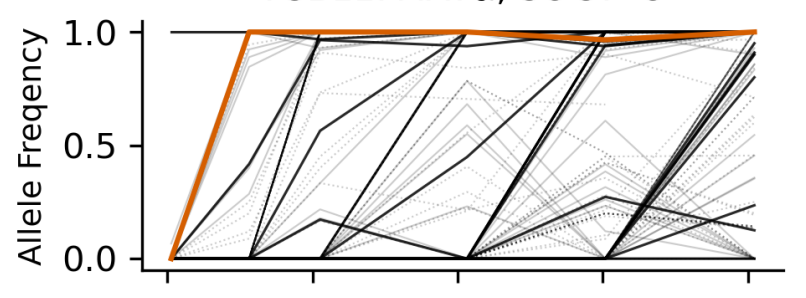

P3D11: $M A T \alpha, \operatorname{SC~} 37^{\circ} \mathrm{C}$

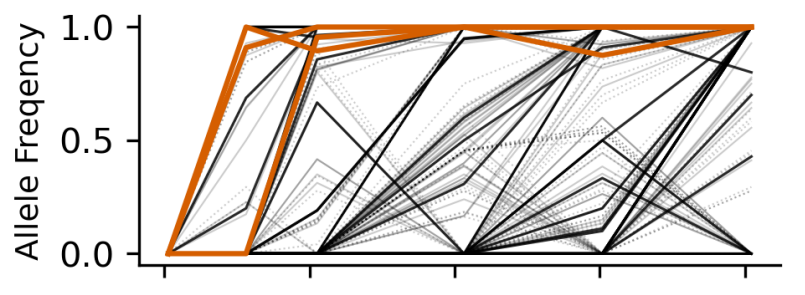

P3F11: $M A T \alpha, S C 37^{\circ} \mathrm{C}$

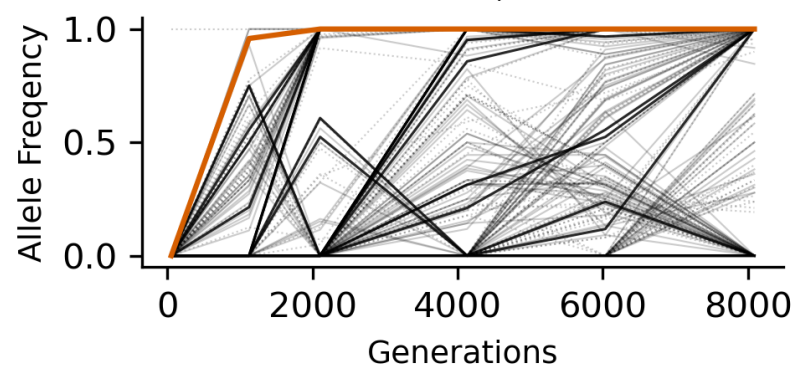

P3C11: $\mathrm{MAT} \alpha, \mathrm{SC} 37^{\circ} \mathrm{C}$

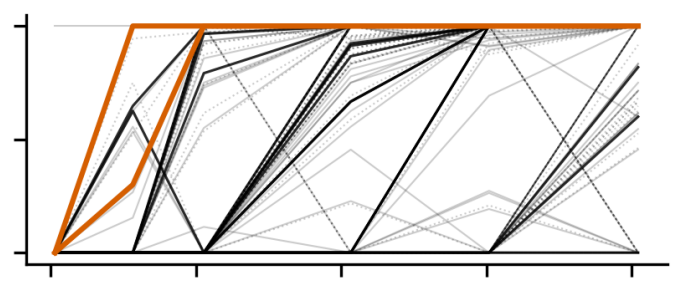

P3E11: $\mathrm{MAT} \alpha, \mathrm{SC} 37^{\circ} \mathrm{C}$

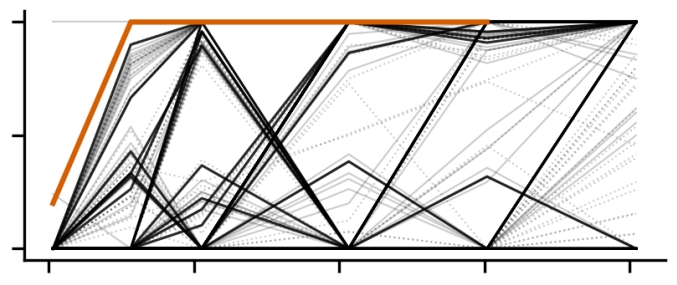

P3G11: MAT $\alpha, \operatorname{SC~} 37^{\circ} \mathrm{C}$

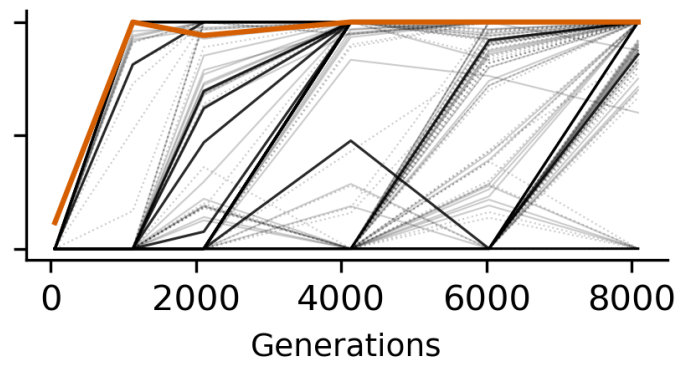

Figure 3 - figure supplement 9. Allele frequencies over time in all focal MAT $\alpha$ populations in SC $37^{\circ} \mathrm{C}$. Nonsynonymous mutations in "multi-hit" genes are solid black lines, mutations in the adenine biosynthesis pathway are colored orange, other nonsynonymous mutations are thin grey lines, and synonymous mutations are dotted lines. 

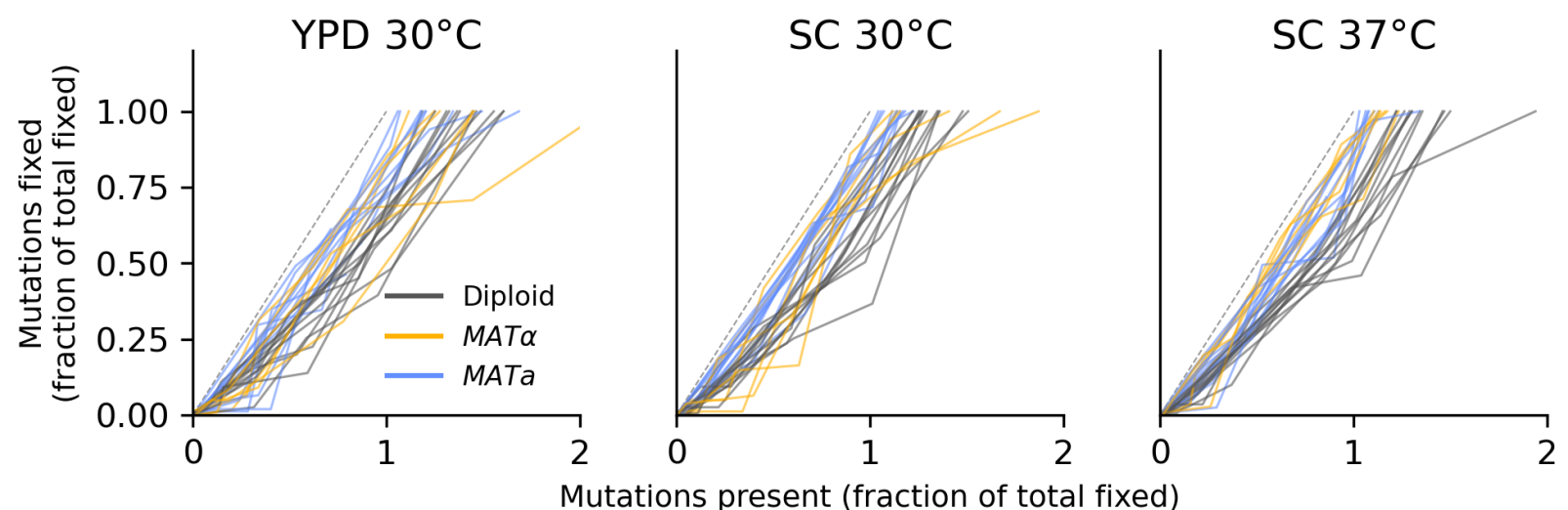

Figure 3 - figure supplement 10. No evidence of coexistence. The number of mutations present in a population plotted against the number of mutations fixed, both scaled by the total number fixed by the final timepoint. Long-term coexistence of multiple lineages in a population would be visible here as horizontal lines because more mutations would be present over time, but no mutations would fix; we do not observe any clear examples of this here, in contrast to the LTEE. 


\begin{tabular}{|c|c|c|c|c|c|}
\hline $\begin{array}{l}\text { Approximate } \\
\text { position }\end{array}$ & $\begin{array}{l}\text { Copy Number } \\
\text { Changes } \\
\text { Observed }\end{array}$ & Type & $\begin{array}{l}\text { Genes likely } \\
\text { affected }\end{array}$ & Notes & Populations with Changes \\
\hline $\begin{array}{l}\text { chrI:19500.. } \\
23000\end{array}$ & Deletion $(1 \mathrm{~N} \rightarrow 0 \mathrm{~N})$ & Deletion & & $\begin{array}{l}\text { likely Ty1-LTR- } \\
\text { associated }\end{array}$ & P1B02;P3E11 \\
\hline $\begin{array}{l}\text { chrIII:151500.. } \\
169000\end{array}$ & $\begin{array}{l}\text { Duplication } \\
(1 \mathrm{~N} \rightarrow 2 \mathrm{~N}) \text {, Deletion } \\
(2 \mathrm{~N} \rightarrow 1 \mathrm{~N})\end{array}$ & $\begin{array}{l}\text { Duplication } \\
\text { \& Deletion } \\
\text { seen }\end{array}$ & $\begin{array}{l}\text { MAK32;PET18;MA } \\
\text { K31;HSP30;SLM5; } \\
\text { PMP1;NPP1;RHB1; }\end{array}$ & $\begin{array}{l}\text { likely Ty1-LTR- } \\
\text { associated }\end{array}$ & P2G04;P3C03;P3D09 \\
\hline $\begin{array}{l}\text { chrIV:1155000.. } \\
1160000\end{array}$ & $\begin{array}{l}\text { Deletion }(1 \mathrm{~N} \rightarrow 0 \mathrm{~N}, \\
2 \mathrm{~N} \rightarrow 0 \mathrm{~N})\end{array}$ & Deletion & HXT6;HXT7 & $\begin{array}{l}\text { likely due to HXT6-HXT7 } \\
\text { recombination }\end{array}$ & $\begin{array}{l}\text { P1B04;P1B11;P1C05;P1C06; } \\
\text { P1E04;P1G04;P1G05;P1G08; } \\
\text { P1B07;P1G10;P2B07 }\end{array}$ \\
\hline $\begin{array}{l}\text { chrIV:1160000.. } \\
1163600\end{array}$ & $\begin{array}{l}\text { Deletion }(1 \mathrm{~N} \rightarrow 0 \mathrm{~N}, \\
2 \mathrm{~N} \rightarrow 0 \mathrm{~N})\end{array}$ & Deletion & HXT3;HXT6 & $\begin{array}{l}\text { likely due to HXT3-HXT6 } \\
\text { recombination }\end{array}$ & $\begin{array}{l}\text { P1C02;P1E09;P1G08;P1B07; } \\
\text { P1G10;P2B07;P2C10 }\end{array}$ \\
\hline $\begin{array}{l}\text { chrIV:884000.. } \\
987500\end{array}$ & $\begin{array}{l}\text { Duplication } \\
(1 \mathrm{~N} \rightarrow 2 \mathrm{~N}, 2 \mathrm{~N} \rightarrow 3 \mathrm{~N})\end{array}$ & Duplication & Many & $\begin{array}{l}\text { likely Ty1/Ty2- } \\
\text { associated }\end{array}$ & P3G09;P3C11 \\
\hline $\begin{array}{l}\text { chrV:497000.. } \\
569500\end{array}$ & $\begin{array}{l}\text { Duplication } \\
(2 \mathrm{~N} \rightarrow 4 \mathrm{~N}, 2 \mathrm{~N} \rightarrow 3 \mathrm{~N})\end{array}$ & Duplication & Many & $\begin{array}{l}\text { duplication of a large } \\
\text { section of the right arm } \\
\text { of chrV, likely Ty1- } \\
\text { associated }\end{array}$ & $\begin{array}{l}\text { P3B08;P3D09;P2B09;P3D10; } \\
\text { P1C07;P1G09;P2F09;P2G10; } \\
\text { P2D08 }\end{array}$ \\
\hline $\begin{array}{l}\text { chrX:198000.. } \\
202000\end{array}$ & $\begin{array}{l}\text { Duplication } \\
(1 \mathrm{~N} \rightarrow 2 \mathrm{~N}) \text {, Deletion } \\
(2 \mathrm{~N} \rightarrow 0 \mathrm{~N}, 1 \mathrm{~N} \rightarrow 0 \mathrm{~N})\end{array}$ & $\begin{array}{l}\text { Duplication } \\
\text { \& Deletion } \\
\text { seen }\end{array}$ & & $\begin{array}{l}\text { likely Ty1/Ty4- } \\
\text { associated }\end{array}$ & P2C06;P2D11;P3C07;P3G06 \\
\hline chrXV:0..25000 & $\begin{array}{l}\text { Duplication } \\
(2 \mathrm{~N} \rightarrow 4 \mathrm{~N})\end{array}$ & Duplication & $\begin{array}{l}\text { IMA2;ENB1;CSS3; } \\
\text { PAU20;BDS1;AAD } \\
15\end{array}$ & $\begin{array}{l}\text { duplication of a large } \\
\text { section of the left arm } \\
\text { of chrXV }\end{array}$ & P3B10;P3D10 \\
\hline $\begin{array}{l}\text { chrXV:1074000.. } \\
1078500\end{array}$ & Deletion $(1 \mathrm{~N} \rightarrow 0 \mathrm{~N})$ & Deletion & HSP33 & & P2D06;P3G06 \\
\hline
\end{tabular}

Figure 3 - figure supplement 11. Table of copy number change events observed in more than one population, excluding those show in Figure 3 - figure supplement 12. All detected copy number change events are listed in Supplementary file 3. 

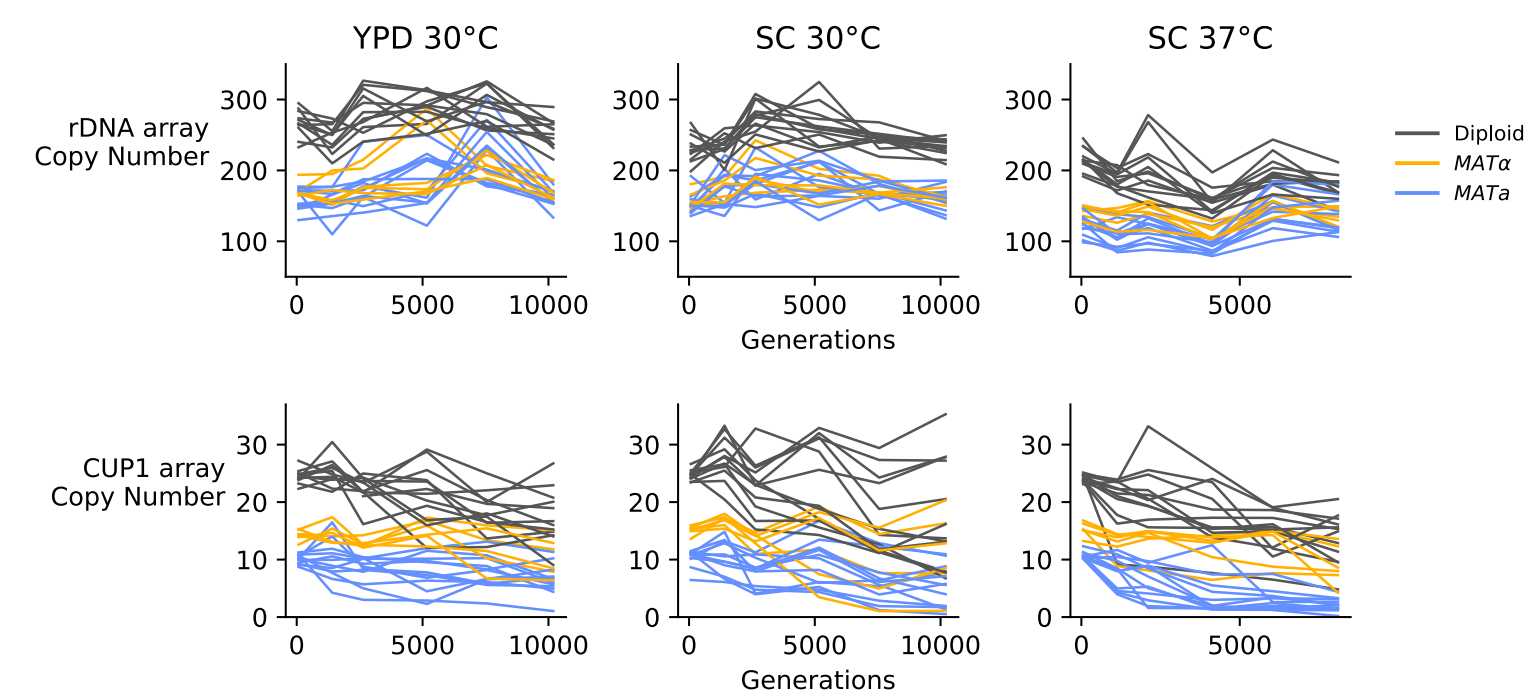

Figure 3 - figure supplement 12. Copy number variation in the ribosomal DNA array and CUP1 array, determined from sequencing coverage data. 
A

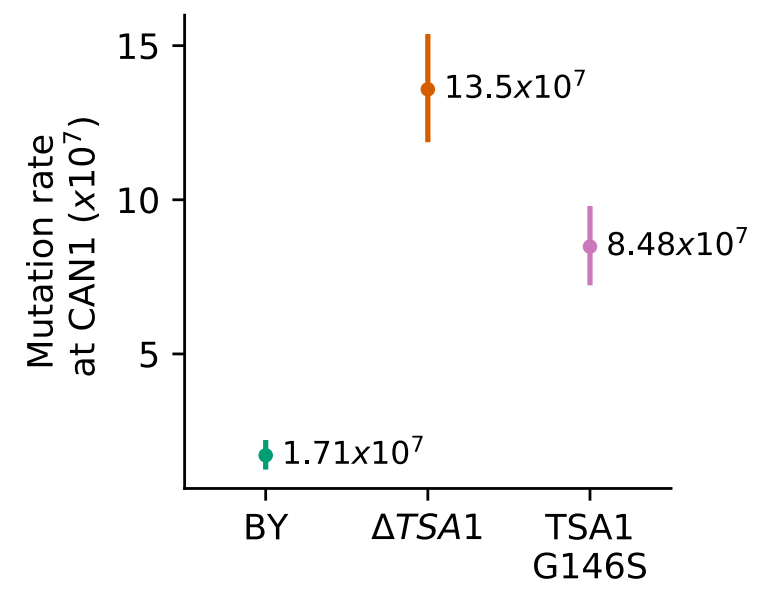

B

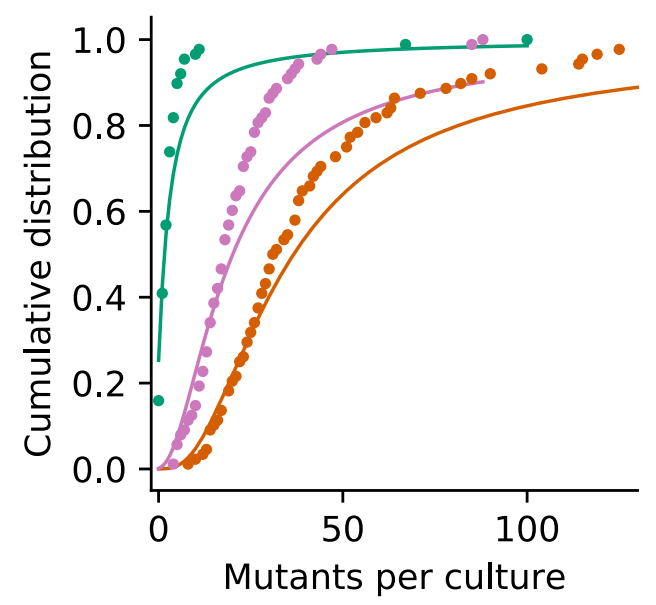

Figure 4 - figure supplement 1. (A) Inferred mutation rates of BY4741, BY4741 with TSA1 deleted (YAN727), and BY4741 with the G146S mutation in TSA1 (YAN728). Error bars represent 95\% confidence intervals. (B) Cumulative distributions of colony counts from fluctuation assays and corresponding Luria-Delbrück fits, colors are the same as in (A). 
A

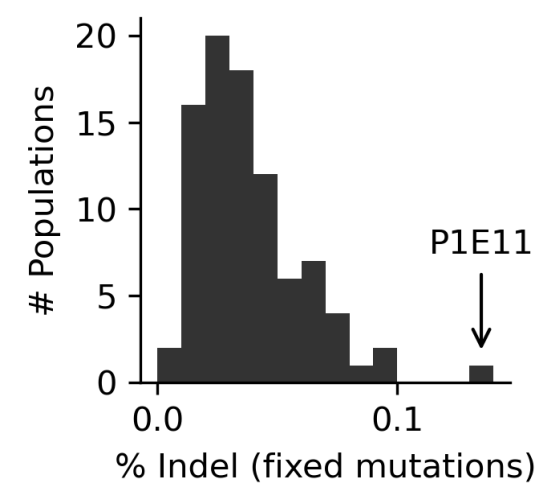

B

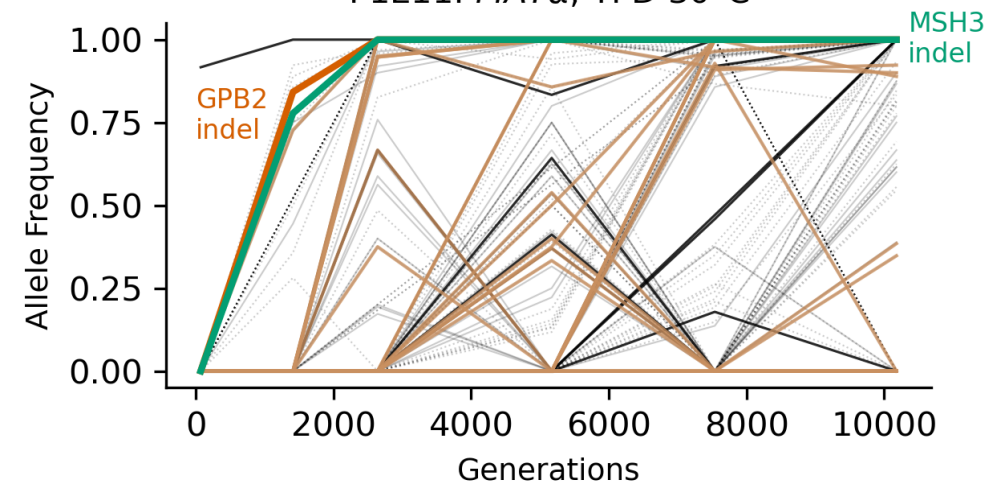

Figure 4 - figure supplement 2. Population P1E11, a putative mutator. (A) Histogram of the percentage of fixed mutations that are indels in all 90 focal populations, with P1E11 indicated by an arrow. (B) Allele frequencies of mutations in P1E11. Nonsynonymous mutations in "multi-hit" genes are solid black lines, other nonsynonymous mutations are thin grey lines, synonymous mutations are dotted lines, indels are brown lines, and mutations in MSH3 and GPB2 are colored and labeled. We hypothesize that the MSH3 mutation hitchhiked to fixation with the selected GPB2 indel mutation, which occurred partially due to higher rates of indel mutations in strains without proper MSH3 function. 

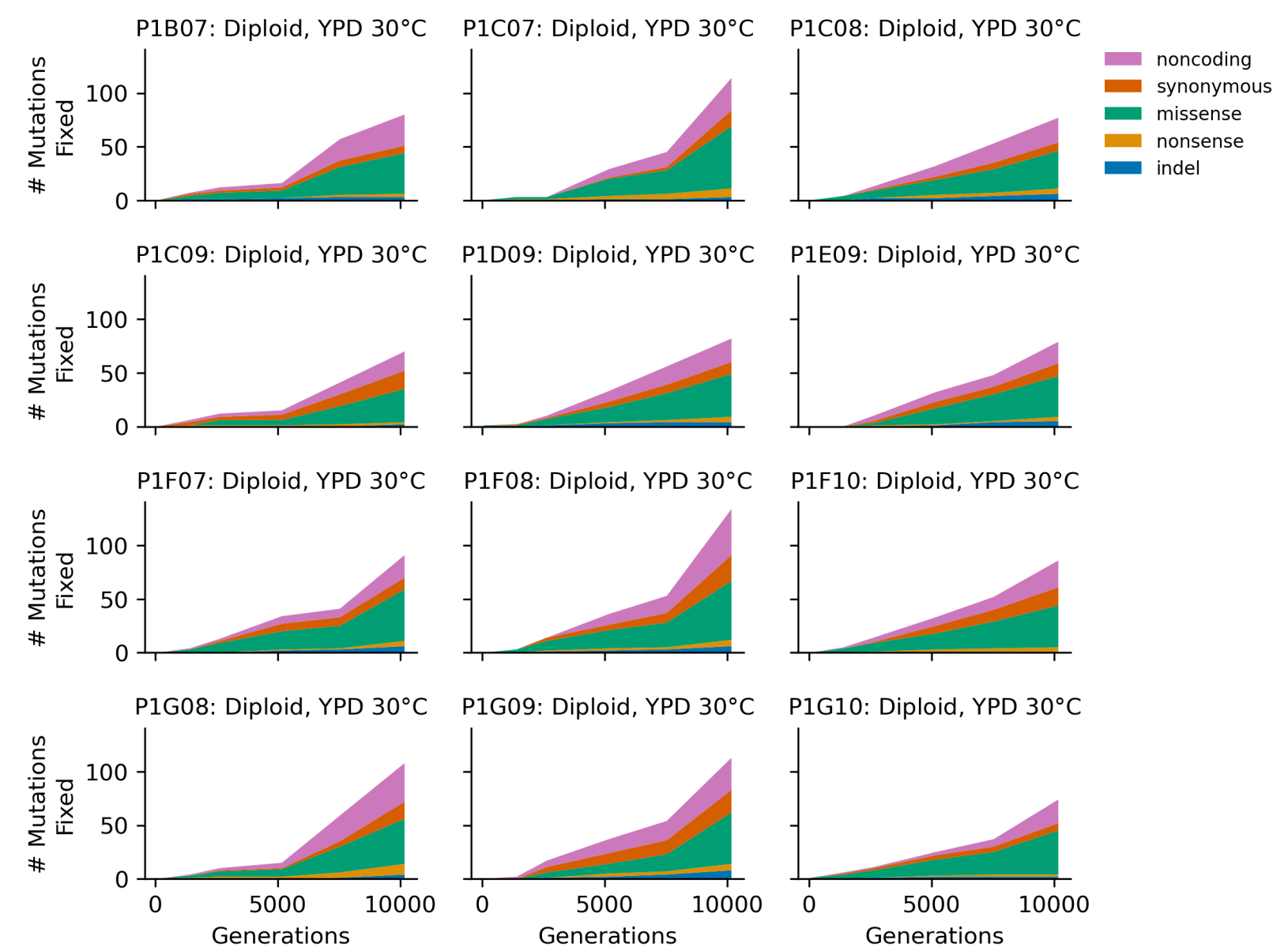

P1E09: Diploid, YPD $30^{\circ} \mathrm{C}$

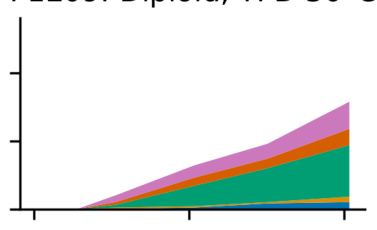

P1F10: Diploid, YPD $30^{\circ} \mathrm{C}$

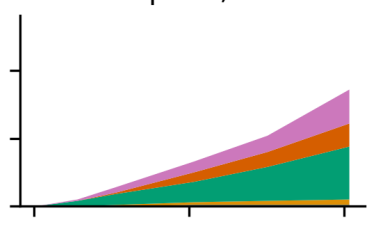

P1G10: Diploid, YPD $30^{\circ} \mathrm{C}$

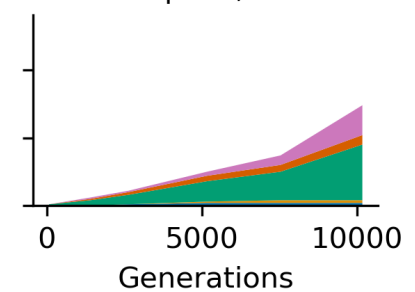

Figure 4 - figure supplement 3. Stacked plot of fixed mutation types over time in all focal diploid populations in $\mathrm{YPD} 30^{\circ} \mathrm{C}$. 

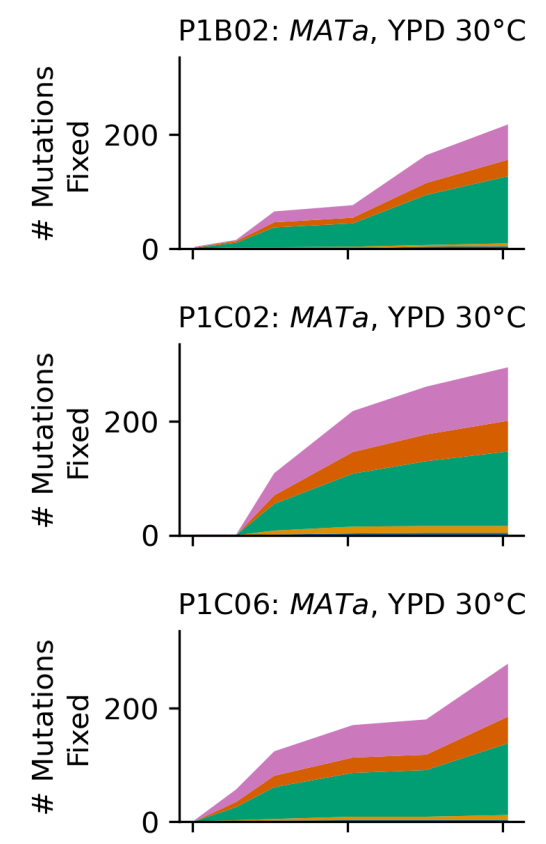

P1F05: MATa, YPD $30^{\circ} \mathrm{C}$

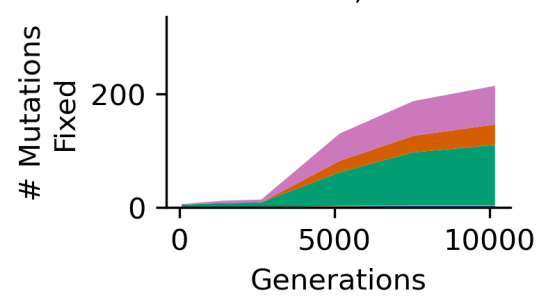

Figure 4 - figure supplement 4. Stacked plot of fixed mutation types over time in all focal MATa populations in YPD $30^{\circ} \mathrm{C}$.
P1B03: MATa, YPD $30^{\circ} \mathrm{C}$

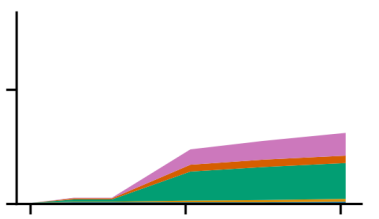

P1C04: MATa, YPD $30^{\circ} \mathrm{C}$

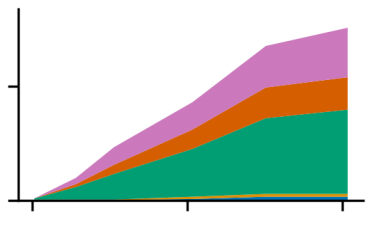

P1D03: MATa, YPD $30^{\circ} \mathrm{C}$
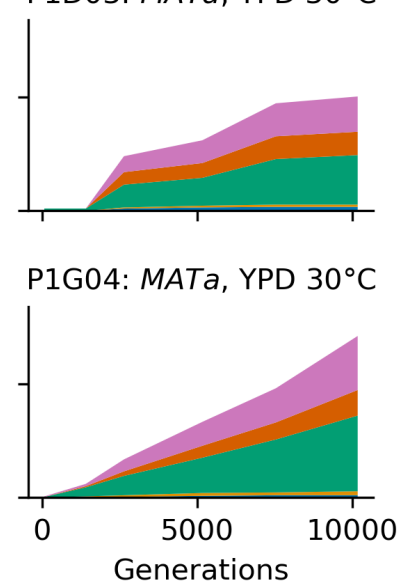

P1B04: MATa, YPD $30^{\circ} \mathrm{C}$

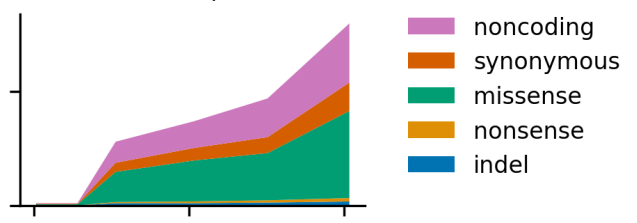

P1C05: MATa, YPD $30^{\circ} \mathrm{C}$

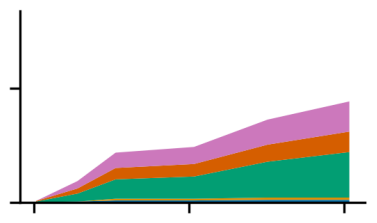

P1E04: MATa, YPD $30^{\circ} \mathrm{C}$

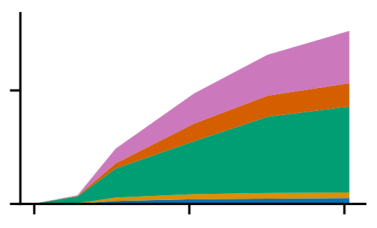

P1G05: MATa, YPD $30^{\circ} \mathrm{C}$

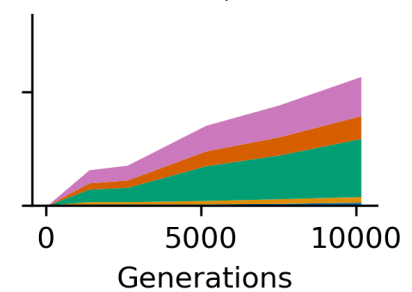



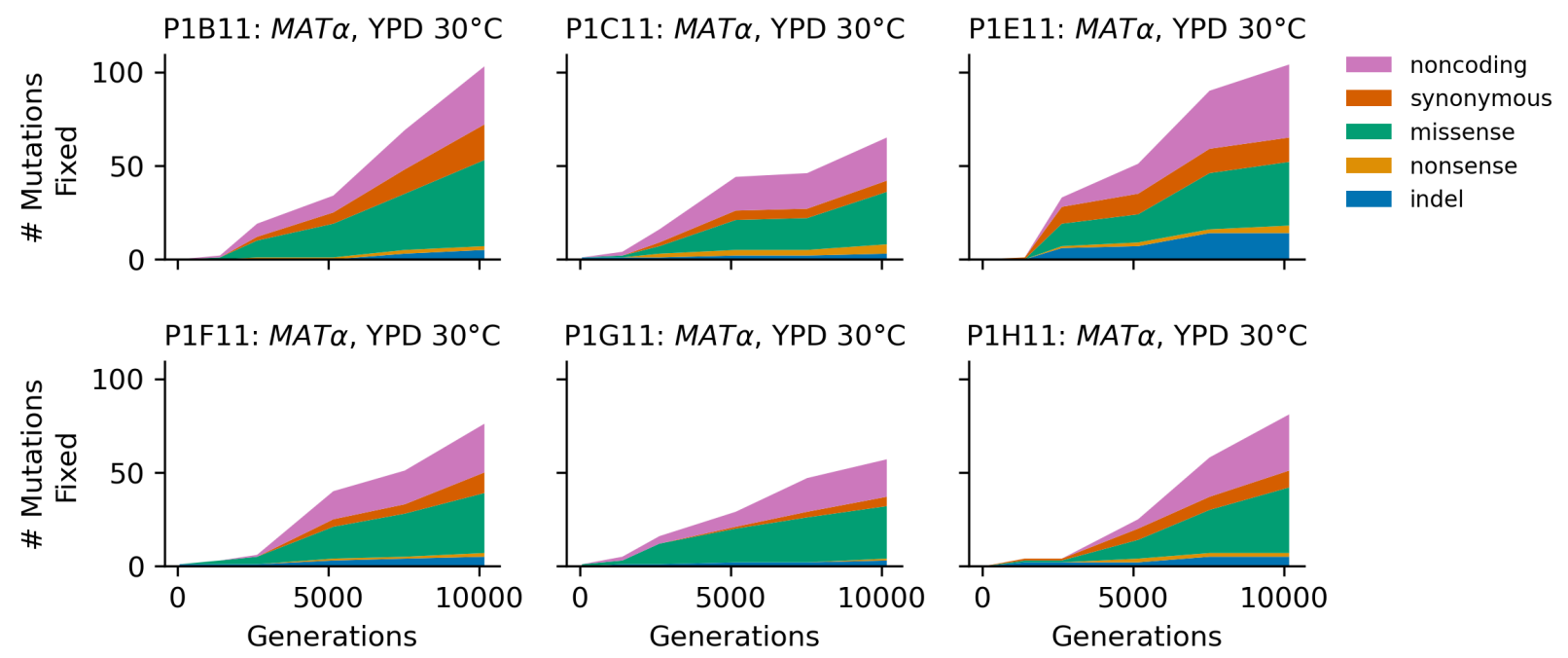

Figure 4 - figure supplement 5. Stacked plot of fixed mutation types over time in all focal MAT $\alpha$ populations in YPD $30^{\circ} \mathrm{C}$. 


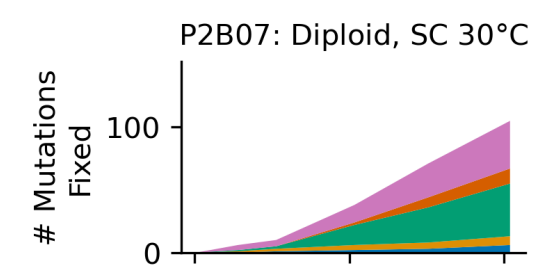

P2B10: Diploid, SC $30^{\circ} \mathrm{C}$

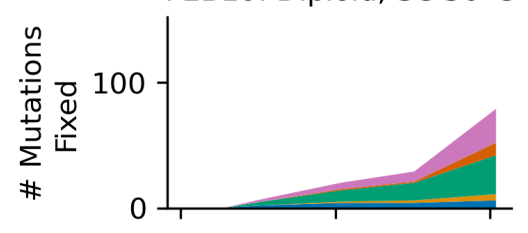

P2E08: Diploid, SC $30^{\circ} \mathrm{C}$

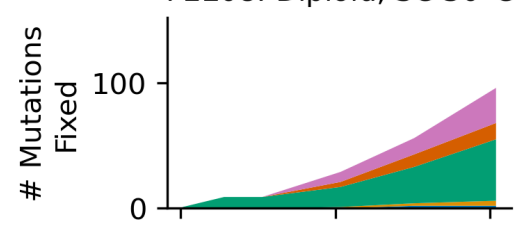

P2G08: Diploid, SC $30^{\circ} \mathrm{C}$

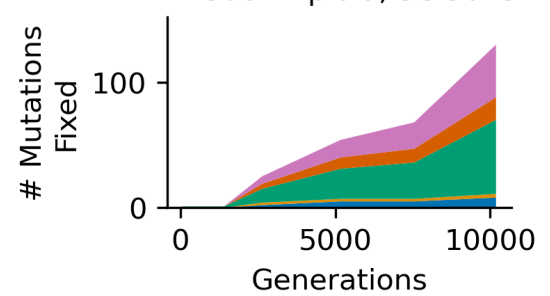

P2B08: Diploid, $\mathrm{SC} 30^{\circ} \mathrm{C}$

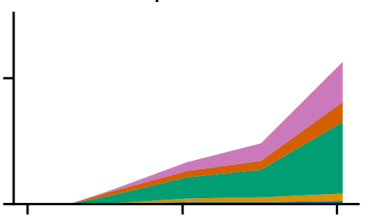

P2C10: Diploid, SC $30^{\circ} \mathrm{C}$

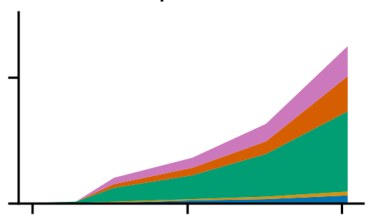

P2F07: Diploid, SC $30^{\circ} \mathrm{C}$

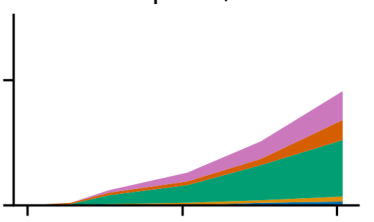

P2G09: Diploid, SC $30^{\circ} \mathrm{C}$

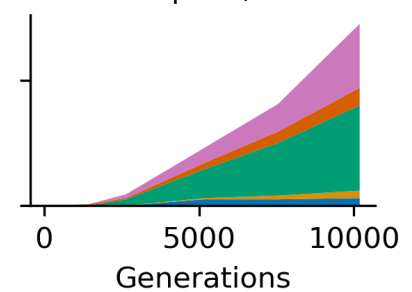

P2B09: Diploid, $\mathrm{SC} 30^{\circ} \mathrm{C}$

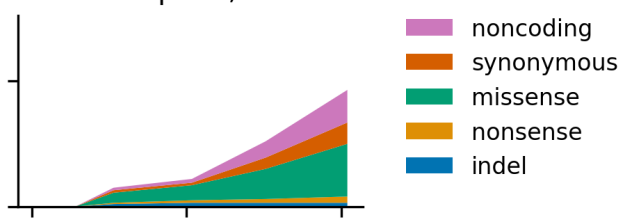

P2D08: Diploid, SC $30^{\circ} \mathrm{C}$

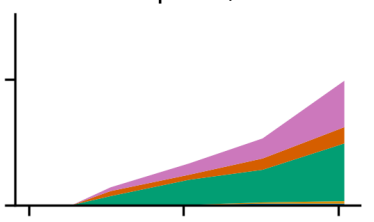

P2F09: Diploid, SC $30^{\circ} \mathrm{C}$

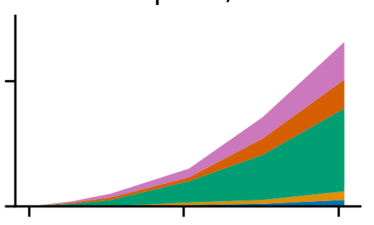

P2G10: Diploid, SC $30^{\circ} \mathrm{C}$

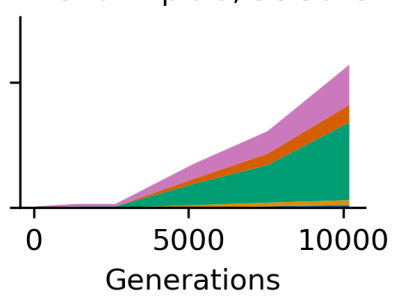

Figure 4 - figure supplement 6. Stacked plot of fixed mutation types over time in all focal diploid populations in $\mathrm{SC} 30^{\circ} \mathrm{C}$. 

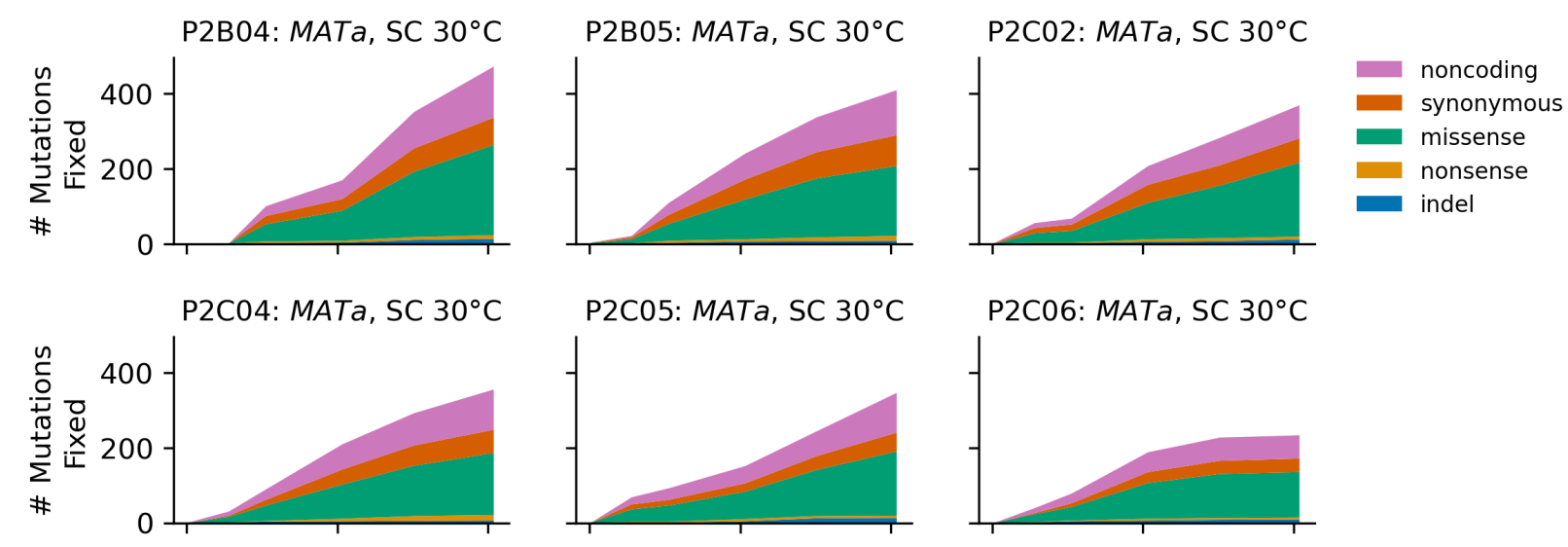

P2C06: MATa, SC $30^{\circ} \mathrm{C}$
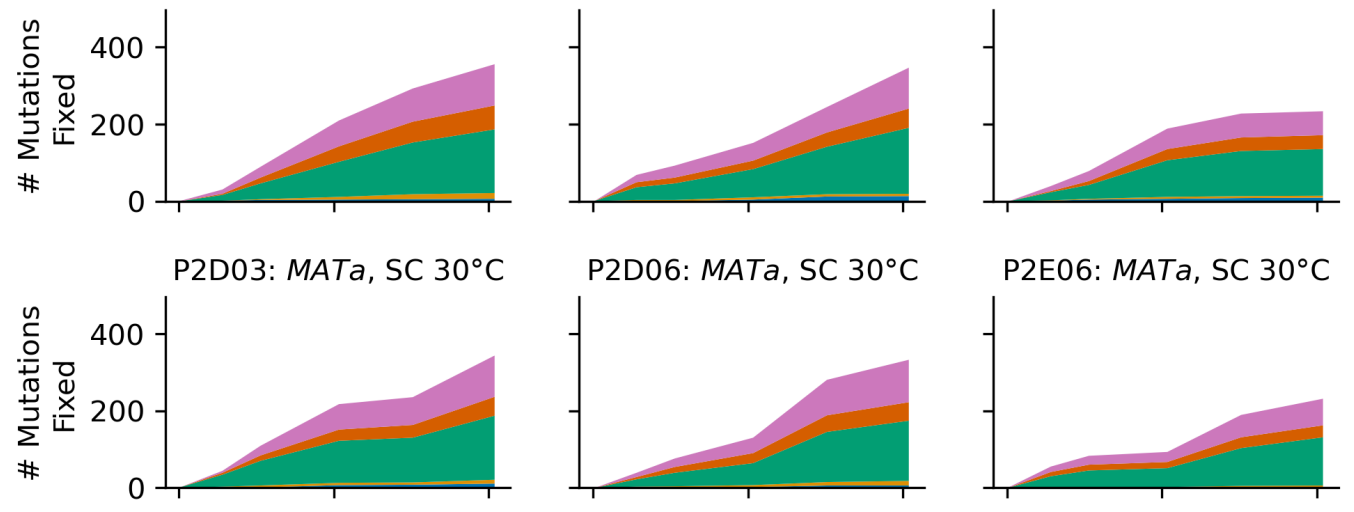

P2F02: MATa, SC $30^{\circ} \mathrm{C}$
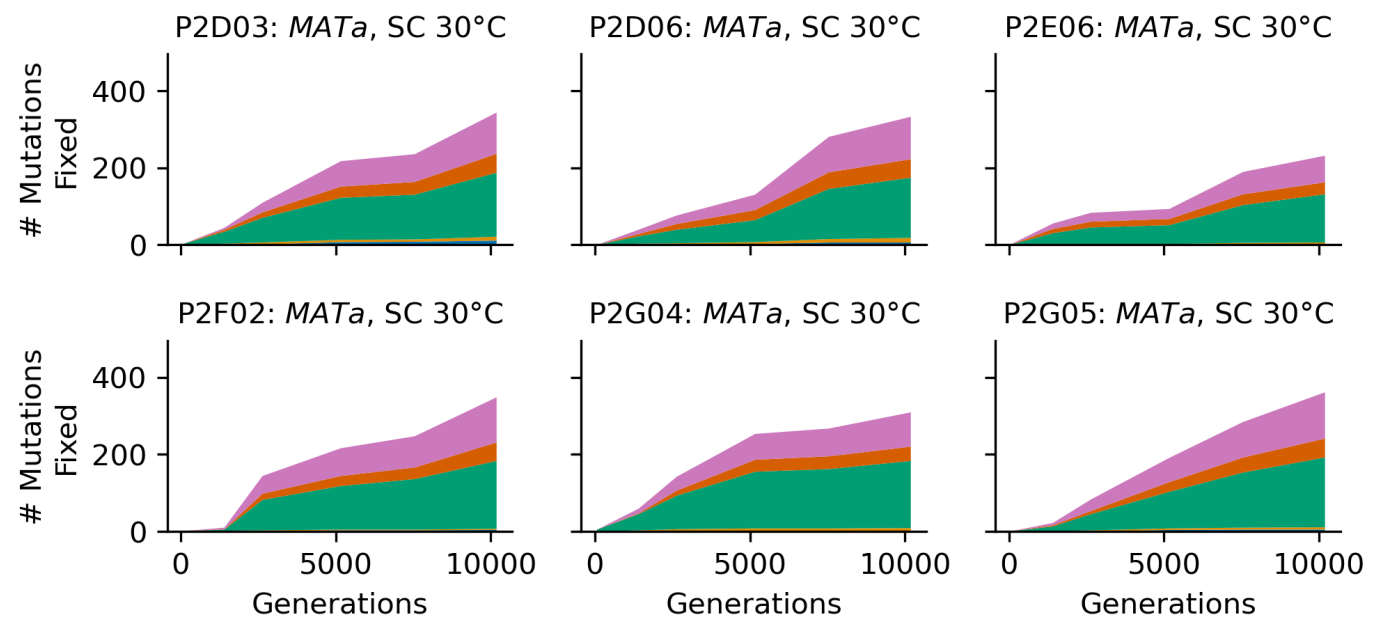

Figure 4 - figure supplement 7. Stacked plot of fixed mutation types over time in all focal MATa populations in $\mathrm{SC} 30^{\circ} \mathrm{C}$. 

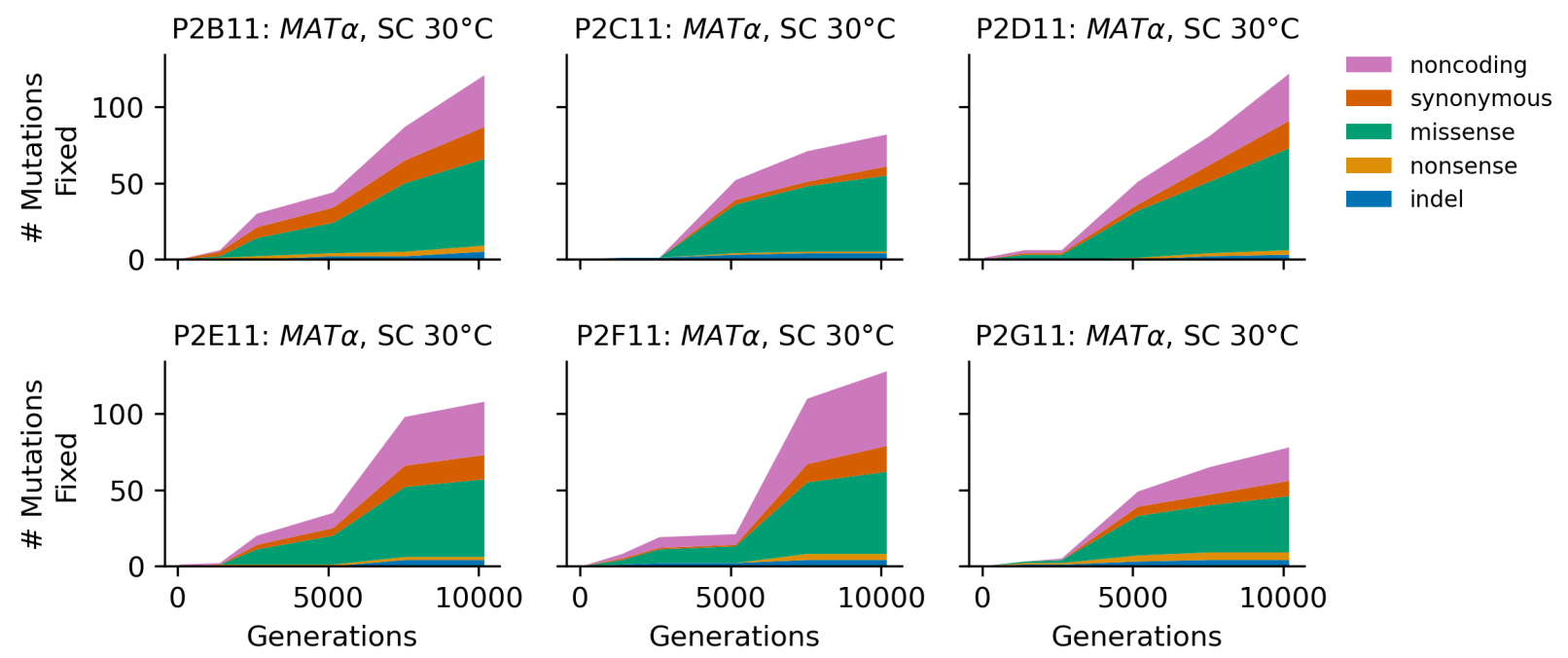

Figure 4 - figure supplement 8. Stacked plot of fixed mutation types over time in all focal MAT $\alpha$ populations in $\mathrm{SC} 30^{\circ} \mathrm{C}$. 

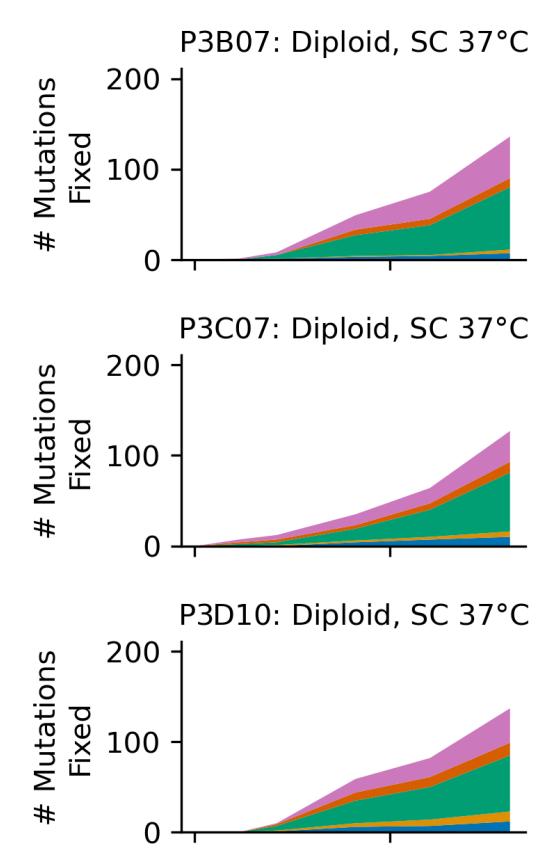

P3F09: Diploid, SC $37^{\circ} \mathrm{C}$

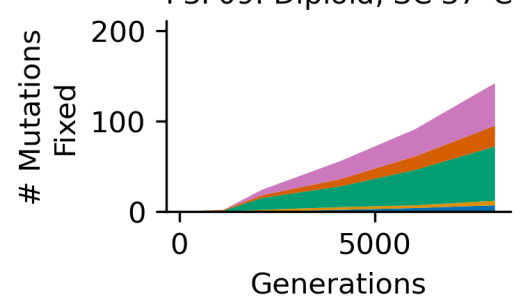

P3B08: Diploid, SC $37^{\circ} \mathrm{C}$

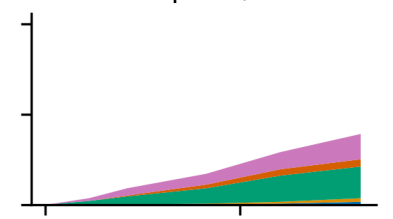

P3C10: Diploid, SC $37^{\circ} \mathrm{C}$

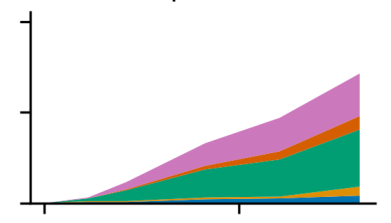

P3E08: Diploid, SC $37^{\circ} \mathrm{C}$

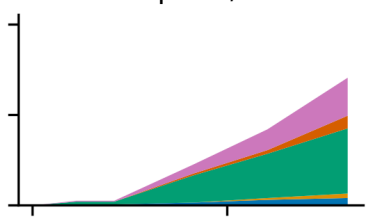

P3G09: Diploid, SC $37^{\circ} \mathrm{C}$

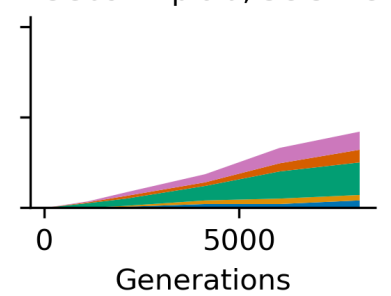

P3B10: Diploid, $5 C 37^{\circ} \mathrm{C}$

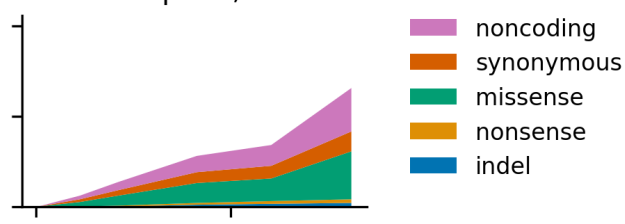

P3D09: Diploid, SC $37^{\circ} \mathrm{C}$

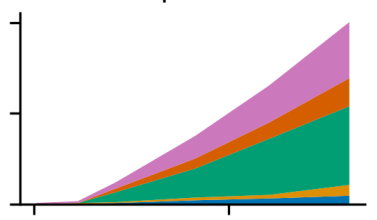

P3F07: Diploid, SC $37^{\circ} \mathrm{C}$

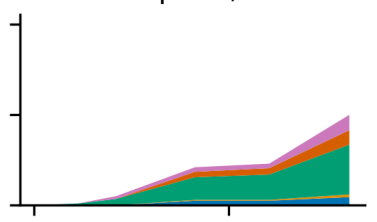

P3G10: Diploid, SC $37^{\circ} \mathrm{C}$

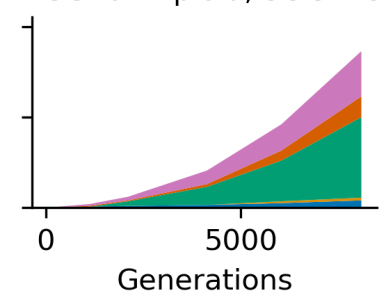

Figure 4 - figure supplement 9. Stacked plot of fixed mutation types over time in all focal diploid populations in $\mathrm{SC} 37^{\circ} \mathrm{C}$. 

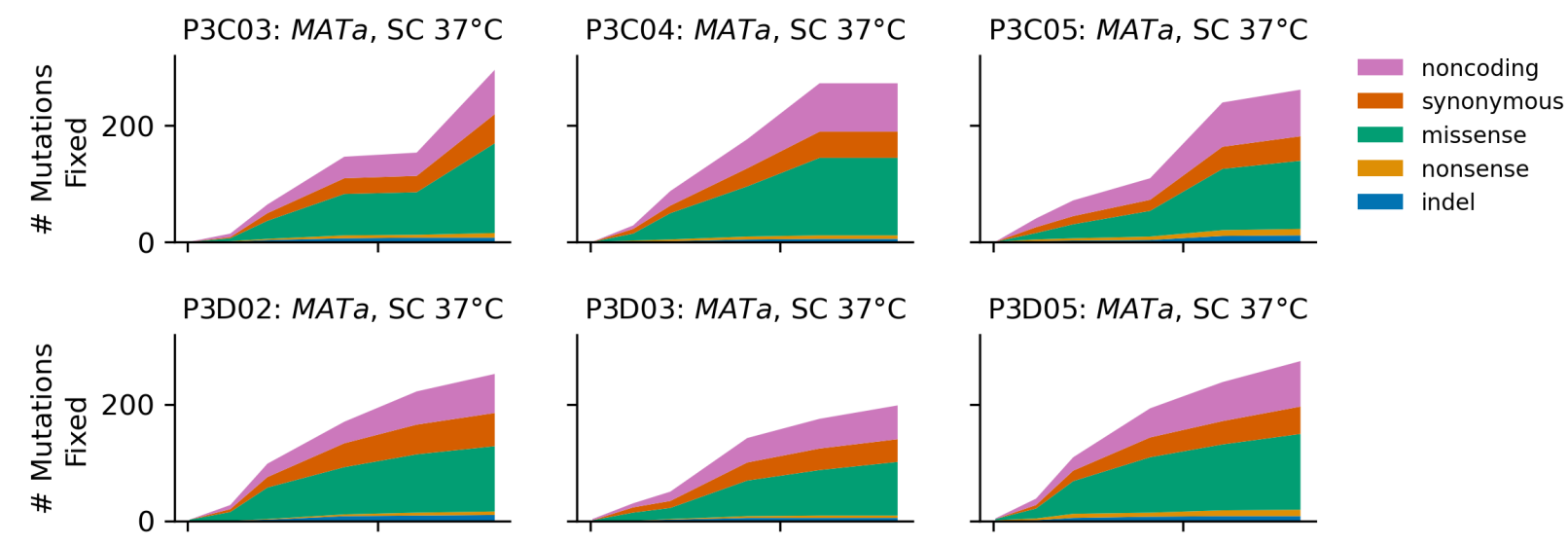

P3D05: MATa, SC $37^{\circ} \mathrm{C}$
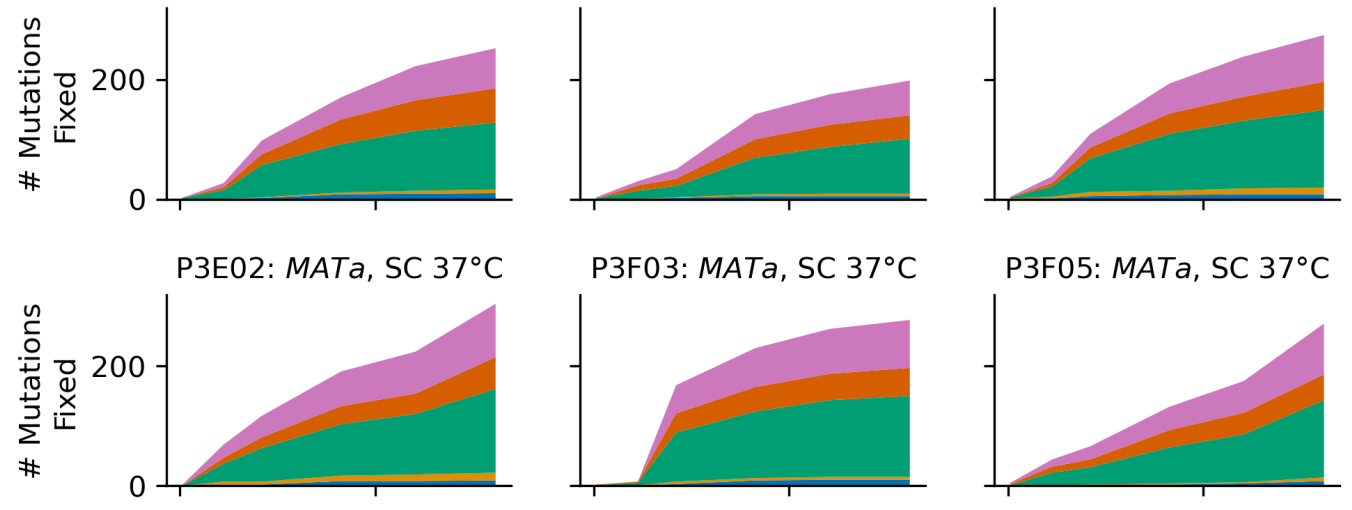

P3F05: MATa, SC $37^{\circ} \mathrm{C}$

P3G02: MATa, SC $37^{\circ} \mathrm{C}$
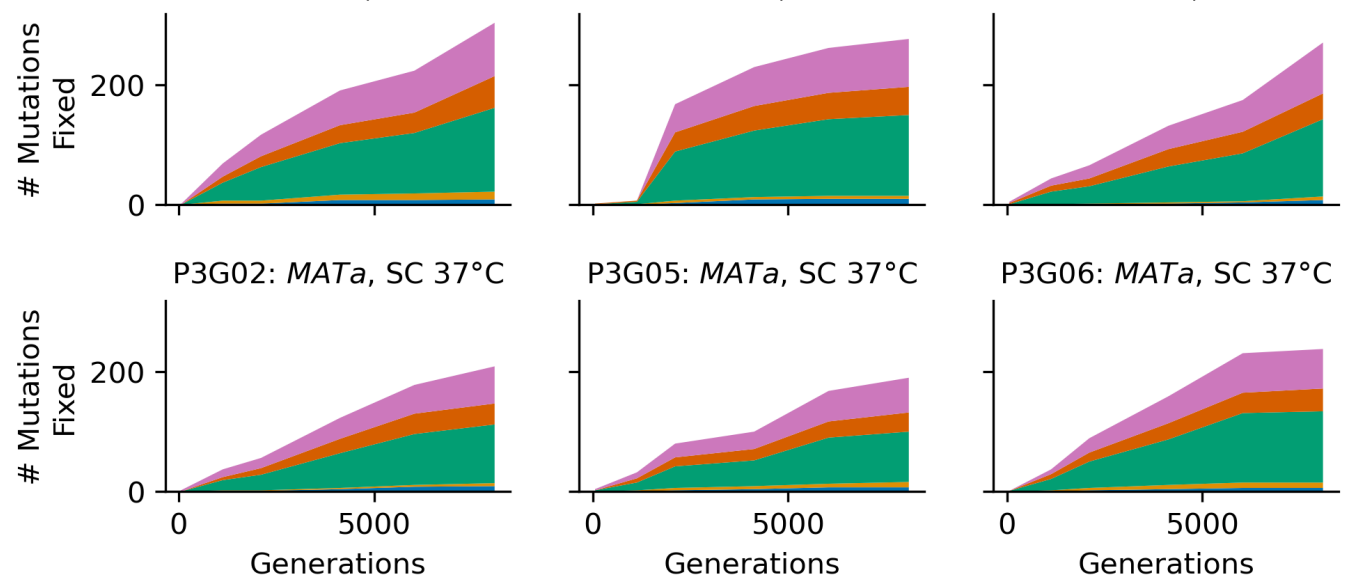

P3G06: $\mathrm{MATa}, \mathrm{SC} 37^{\circ} \mathrm{C}$

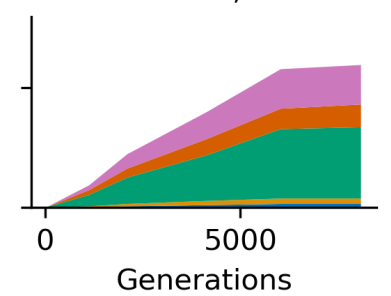

Figure 4 - figure supplement 10. Stacked plot of fixed mutation types over time in all focal MATa populations in $\mathrm{SC} 37^{\circ} \mathrm{C}$. 

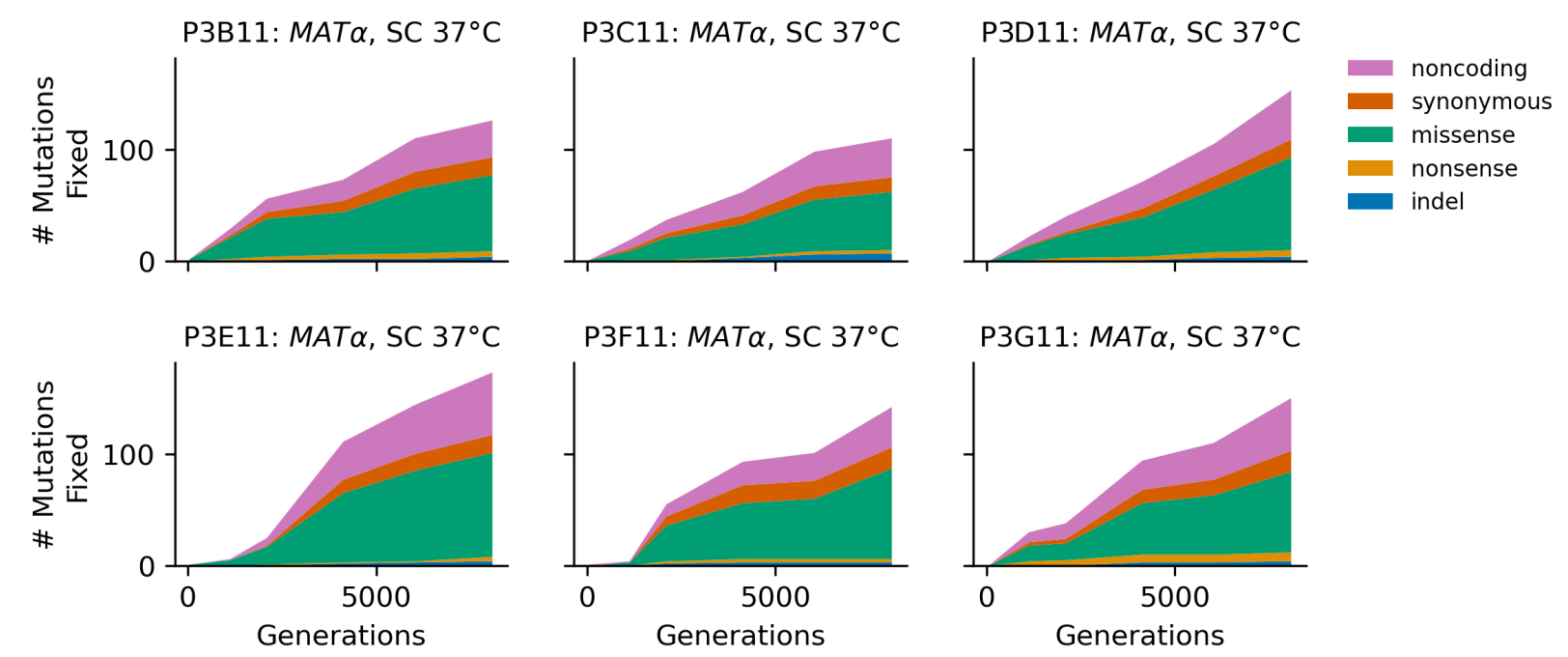

Figure 4 - figure supplement 11. Stacked plot of fixed mutation types over time in all focal MAT $\alpha$ populations in $\mathrm{SC} 37^{\circ} \mathrm{C}$. 
bioRxiv preprint doi: https://doi.org/10.1101/2020.10.09.330191; this version posted October 10, 2020. The copyright holder for this preprint (which was not certified by peer review) is the author/funder, who has granted bioRxiv a license to display the preprint in perpetuity. It is made available under aCC-BY-ND 4.0 International license.

\begin{tabular}{|c|c|c|c|c|c|c|}
\hline Gene & Pop. & Chrom. & Pos. & Mutation & Change & Notes \\
\hline RRT15 & P1B04 & chrXII & 491006 & $A->G$ & D13G & \multirow{4}{*}{$\begin{array}{l}\text { Possibly the result of homologous } \\
\text { repair; two sequences homologous } \\
\text { to this region have this } A->G \\
\text { mutation, but also have another } \\
\text { mutation not seen in alignments } \\
\text { supporting this mutation (suggesting } \\
\text { this is not just misalignment). }\end{array}$} \\
\hline RRT15 & P3C05 & chrXII & 491006 & $A->G$ & D13G & \\
\hline RRT15 & P3D10 & chrXII & 491006 & $A->G$ & D13G & \\
\hline RRT15 & P2D06 & chrXII & 491006 & $A->G$ & D13G & \\
\hline HHF2 & $\mathrm{P} 1 \mathrm{H} 11$ & chrXIV & 577236 & TTCGGTGGTTAAACAA->T & Frameshift & \multirow{11}{*}{ Repetitive sequence deletion. } \\
\hline HHF2 & P3F07 & chrXIV & 577236 & TTCGGTGGTTAAACAA->T & Frameshift & \\
\hline HHF2 & P3F03 & chrXIV & 577236 & TTCGGTGGTTAAACAA->T & Frameshift & \\
\hline HHF2 & P3C10 & chrXIV & 577236 & TTCGGTGGTTAAACAA->T & Frameshift & \\
\hline HHF2 & P2C11 & chrXIV & 577236 & TTCGGTGGTTAAACAA->T & Frameshift & \\
\hline HHF2 & P1E04 & chrXIV & 577236 & TTCGGTGGTTAAACAA->T & Frameshift & \\
\hline HHF2 & P2B04 & chrXIV & 577236 & TTCGGTGGTTAAACAA->T & Frameshift & \\
\hline HHF2 & P1G08 & chrXIV & 577236 & TTCGGTGGTTAAACAA->T & Frameshift & \\
\hline HHF2 & $\mathrm{P} 2 \mathrm{C} 10$ & chrXIV & 577236 & TTCGGTGGTTAAACAA->T & Frameshift & \\
\hline HHF2 & P1B07 & chrXIV & 577236 & TTCGGTGGTTAAACAA->T & Frameshift & \\
\hline HHF2 & P3G10 & chrXIV & 577236 & TTCGGTGGTTAAACAA->T & Frameshift & \\
\hline ADE5,7 & P1B11 & chrVII & 57654 & $A->G$ & R399G & \multirow{3}{*}{$\begin{array}{l}\text { Possibly functional, possibly by } \\
\text { chance since this is a multi-hit gene. }\end{array}$} \\
\hline ADE5,7 & P3B07 & chrVII & 57655 & $G->A$ & R399K & \\
\hline ADE5,7 & P3E02 & chrVII & 57655 & $G->T$ & R399I & \\
\hline ADE2 & P1E11 & chrXV & 565924 & $A->C$ & Stop64E & \multirow{5}{*}{$\begin{array}{l}\text { Parallelism due to strong fitness } \\
\text { benefit of fixing the premature stop } \\
\text { codon ade2-1. }\end{array}$} \\
\hline ADE2 & P2F07 & chrXV & 565922 & $\mathrm{~T}->\mathrm{G}$ & Stop64Y & \\
\hline ADE2 & P2B09 & chrXV & 565924 & $A->G$ & Stop64Q & \\
\hline ADE2 & P1C09 & chrXV & 565924 & $A->G$ & Stop64Q & \\
\hline ADE2 & P3B10 & chrXV & 565924 & $A->C$ & Stop64E & \\
\hline ADE2 & P2D01 & chrXV & 565924 & $A->T$ & Stop64K & $\begin{array}{l}\text { Non-focal population, mutation } \\
\text { predicted by fitness data and } \\
\text { confirmed by Sanger sequencing }\end{array}$ \\
\hline ACE2 & P3C07 & chrXII & 406273 & AT->A & Frameshift & \multirow{3}{*}{ T deletion in an $8 \mathrm{~T}$ run. } \\
\hline ACE2 & P1E09 & chrXII & 406273 & AT->A & Frameshift & \\
\hline ACE2 & P3F09 & chrXII & 406273 & AT->A & Frameshift & \\
\hline CCW12 & P2B07 & chrXII & 370261 & TACA->T & Frameshift & \multirow{4}{*}{$\begin{array}{l}\text { Repetitive sequence deletion; yields a } \\
\text { protein with one less Leucine at the } \\
\text { end of the protein. }\end{array}$} \\
\hline CCW12 & P3B07 & chrXII & 370261 & TACA->T & Frameshift & \\
\hline CCW12 & P3E08 & chrXII & 370261 & TACA->T & Frameshift & \\
\hline CCW12 & P1F07 & chrXII & 370261 & TACA->T & Frameshift & \\
\hline STE12 & P3F11 & chrVIII & 276224 & $\mathrm{TG}->\mathrm{T}$ & Frameshift & \multirow{7}{*}{$\mathrm{G}$ deletion or insertion in an $8 \mathrm{G}$ run. } \\
\hline STE12 & P3D05 & chrVIII & 276224 & TG->T & Frameshift & \\
\hline STE12 & P1G09 & chrVIII & 276224 & TG->T & Frameshift & \\
\hline STE12 & P3F05 & chrVIII & 276224 & $\mathrm{TG}->\mathrm{T}$ & Frameshift & \\
\hline STE12 & P3D03 & chrVIII & 276224 & TG->TGG & Frameshift & \\
\hline STE12 & P3G02 & chrVIII & 276224 & $\mathrm{TG}->\mathrm{T}$ & Frameshift & \\
\hline STE12 & P3C11 & chrVIII & 276224 & TG->T & Frameshift & \\
\hline
\end{tabular}

Figure 5 - figure supplement 1. All fixed mutations in codon positions with 3 or more fixed mutations. 
bioRxiv preprint doi: https://doi.org/10.1101/2020.10.09.330191; this version posted October 10,2020 . The copyright holder for this preprint (which was not certified by peer review) is the author/funder, who has granted bioRxiv a license to display the preprint in perpetuity. It is made available under aCC-BY-ND 4.0 International license.

\begin{tabular}{|c|c|c|c|c|}
\hline GO ID & GO term & $\begin{array}{c}\text { P-value } \\
\text { (Benjamini- } \\
\text { Hochberg } \\
\text { Corrected) }\end{array}$ & $\begin{array}{l}\text { Number } \\
\text { genes in } \\
\text { group }\end{array}$ & Genes Hit in Group \\
\hline GO:0043087 & $\begin{array}{l}\text { regulation of GTPase } \\
\text { activity }\end{array}$ & 0.00012 & 9 & TRS130;IRA1;IQG1;RGA2;TRS120;IRA2 \\
\hline GO:0010525 & $\begin{array}{l}\text { regulation of } \\
\text { transposition, RNA- } \\
\text { mediated }\end{array}$ & 0.0124 & 6 & STE11;STE4;STE5;STE7 \\
\hline GO:0007165 & signal transduction & 0.0124 & 65 & $\begin{array}{l}\text { IRA1;BEM2;STE4;IQG1;RGA2;CYR1;IRA } \\
\text { 2;GPB1;COS111;GPB2 }\end{array}$ \\
\hline GO:0046580 & $\begin{array}{l}\text { negative regulation of } \\
\text { Ras protein signal } \\
\text { transduction }\end{array}$ & 0.0124 & 7 & GPB1;IRA1;IRA2;GPB2 \\
\hline GO:0001403 & $\begin{array}{l}\text { invasive growth in } \\
\text { response to glucose } \\
\text { limitation }\end{array}$ & 0.0124 & 43 & $\begin{array}{l}\text { STE12;STE11;STE4;STE5;STE7;GPB1;GP } \\
\text { B2;FLO11 }\end{array}$ \\
\hline GO:0007124 & pseudohyphal growth & 0.0124 & 56 & $\begin{array}{l}\text { STE12;STE11;MDS3;PDA1;STE7;CDC39; } \\
\text { GPB1;GPB2;FLO11 }\end{array}$ \\
\hline GO:0006189 & $\begin{array}{l}\text { de novo' IMP } \\
\text { biosynthetic process }\end{array}$ & 0.03114 & 9 & ADE6;ADE4;ADE2;ADE5,7 \\
\hline GO:0006075 & $\begin{array}{l}(1->3) \text {-beta-D-glucan } \\
\text { biosynthetic process }\end{array}$ & 0.03372 & 4 & GSC2;FKS1;FKS3 \\
\hline GO:0006164 & $\begin{array}{l}\text { purine nucleotide } \\
\text { biosynthetic process }\end{array}$ & 0.03806 & 18 & ADE4;ADE2;ADE5,7;ADE6;ADE3 \\
\hline
\end{tabular}

Figure 6 - figure supplement 1. All GO-enrichments for multi-hit genes that were significant at the $P<0.05$ level after Benjamini-Hochberg multiple hypothesis testing correction. 
bioRxiv preprint doi: https://doi org/10.1101/2020.10.09.330191; this version posted October 10,2020. The copyright holder for this preprint (which was not certified by peer review) is the author/funder, who has granted bioRxiv a license to display the preprint in perpetuity. It is made available under aCC-BY-ND 4.0 International license.
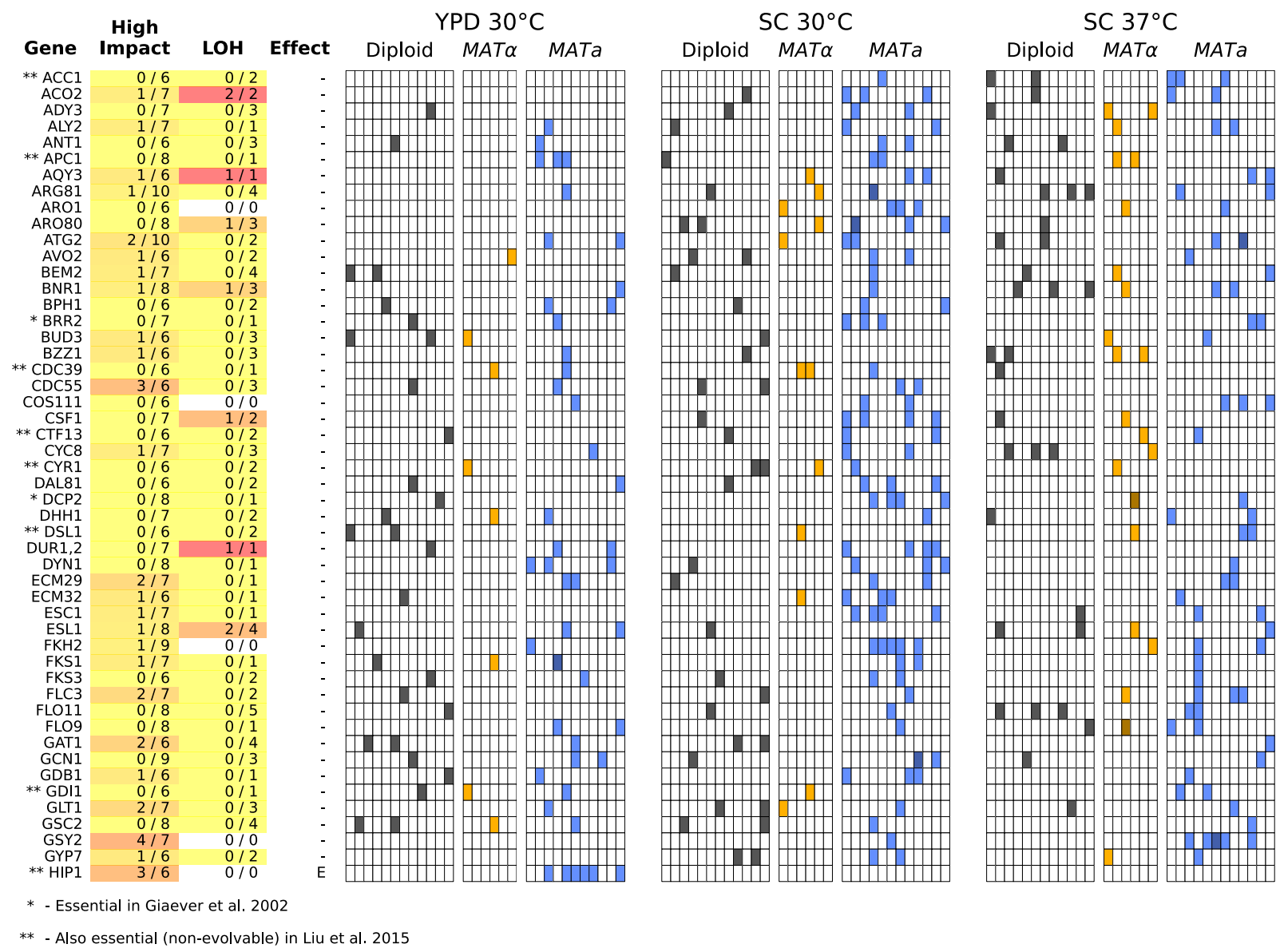

Figure 6 - figure supplement 2. Same as Figure 6, but for all multi-hit genes not shown in Figure 6 (plot 1/3). 
bioRxiv preprint doi: https://doi org/10.1101/2020.10.09.330191; this version posted October 10,2020. The copyright holder for this preprint (which was not certified by peer review) is the author/funder, who has granted bioRxiv a license to display the preprint in perpetuity. It is made available under aCC-BY-ND 4.0 International license.

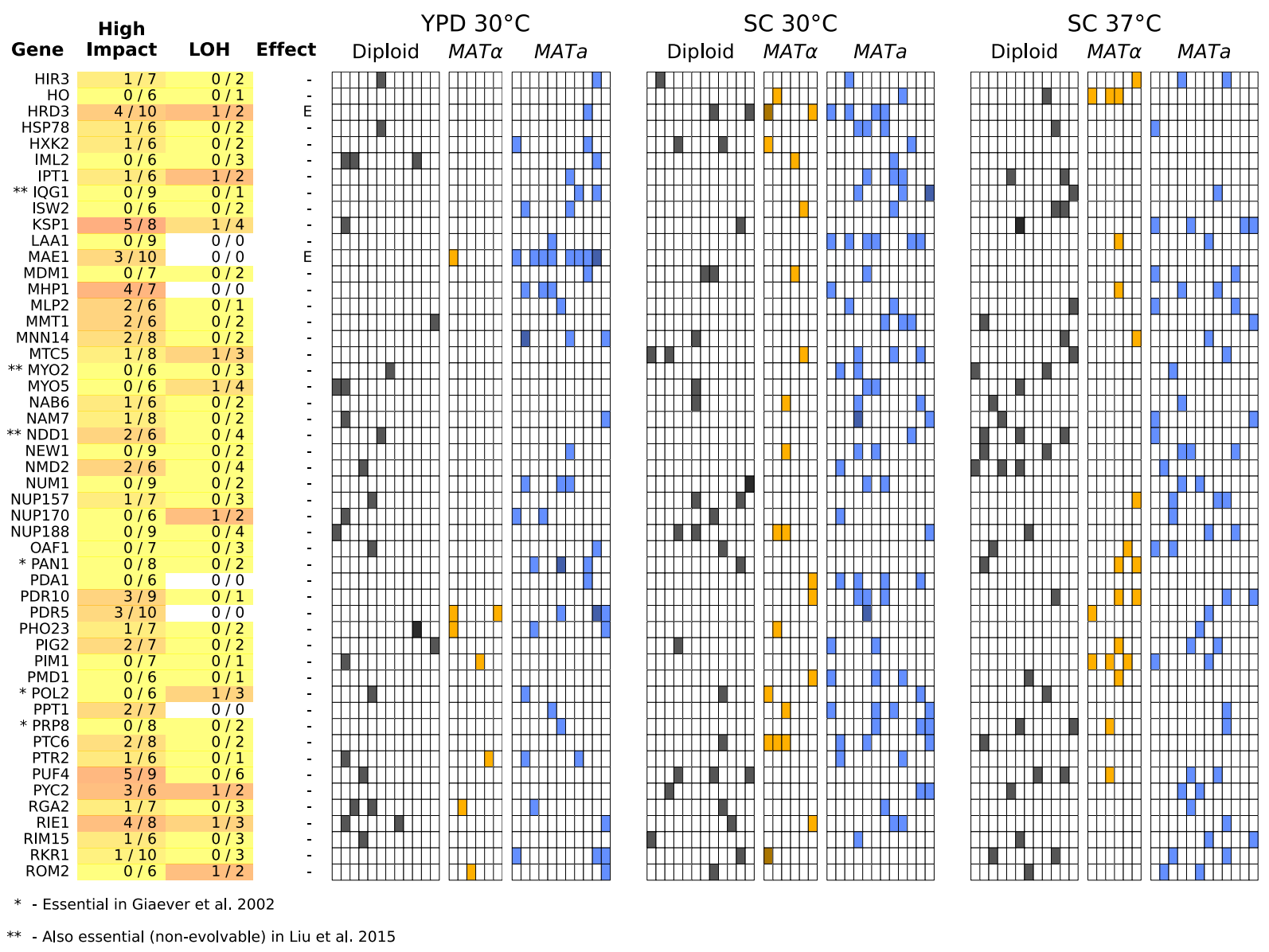

Figure 6 - figure supplement 3. Same as Figure 6, but for all multi-hit genes not shown in Figure 6 (plot 2/3). 
bioRxiv preprint doi: https://doi org/10.1101/2020.10.09.330191; this version posted October 10,2020. The copyright holder for this preprint (which was not certified by peer review) is the author/funder, who has granted bioRxiv a license to display the preprint in perpetuity. It is made available under aCC-BY-ND 4.0 International license.
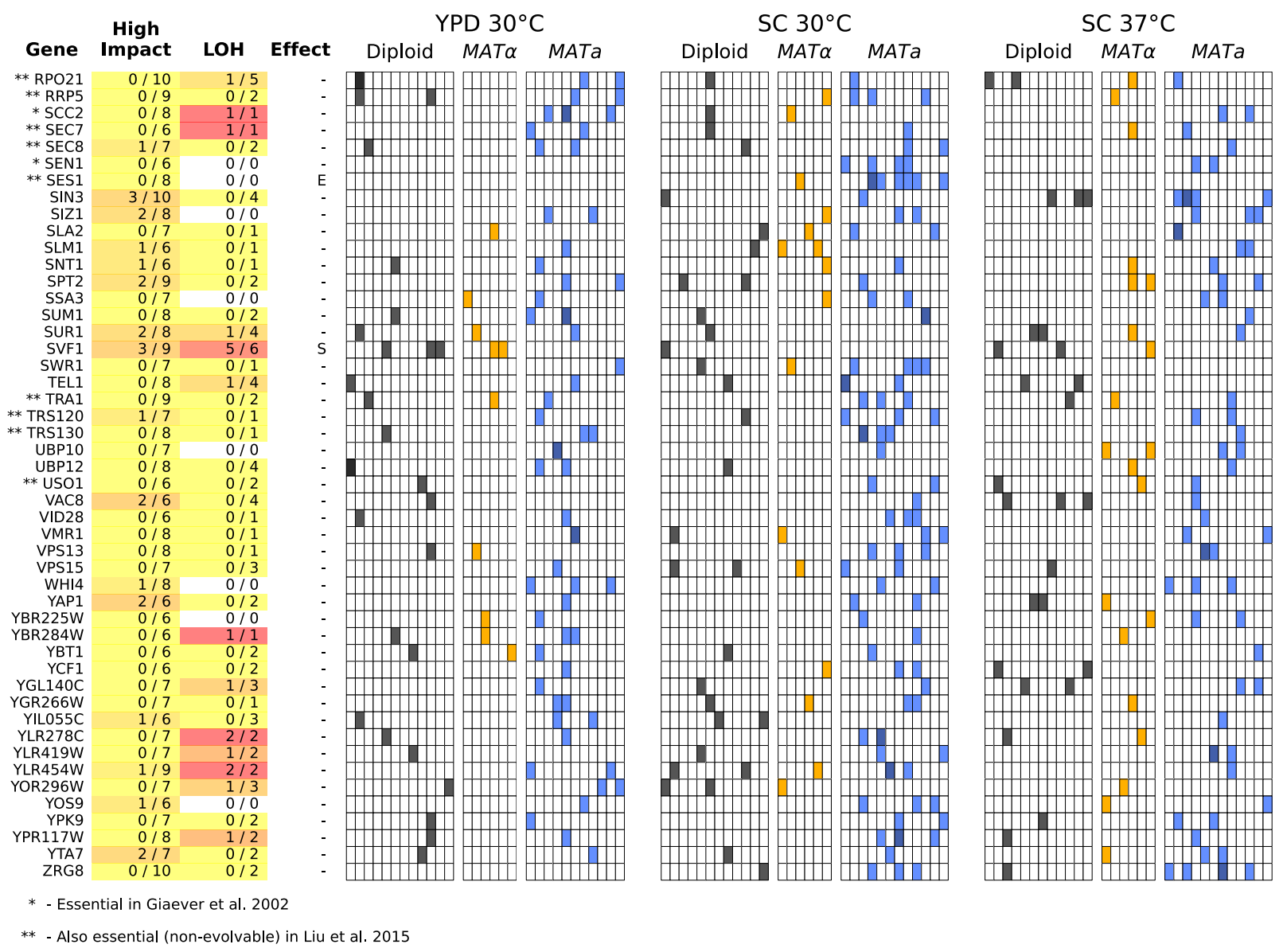

Figure 6 - figure supplement 4. Same as Figure 6, but for all multi-hit genes not shown in Figure 6 (plot 3/3). 
bioRxiv preprint doi: https://doi.org/10.1101/2020.10.09.330191; this version posted October 10, 2020. The copyright holder for this preprint (which was not certified by peer review) is the author/funder, who has granted bioRxiv a license to display the preprint in perpetuity. It is made available under aCC-BY-ND 4.0 International license.
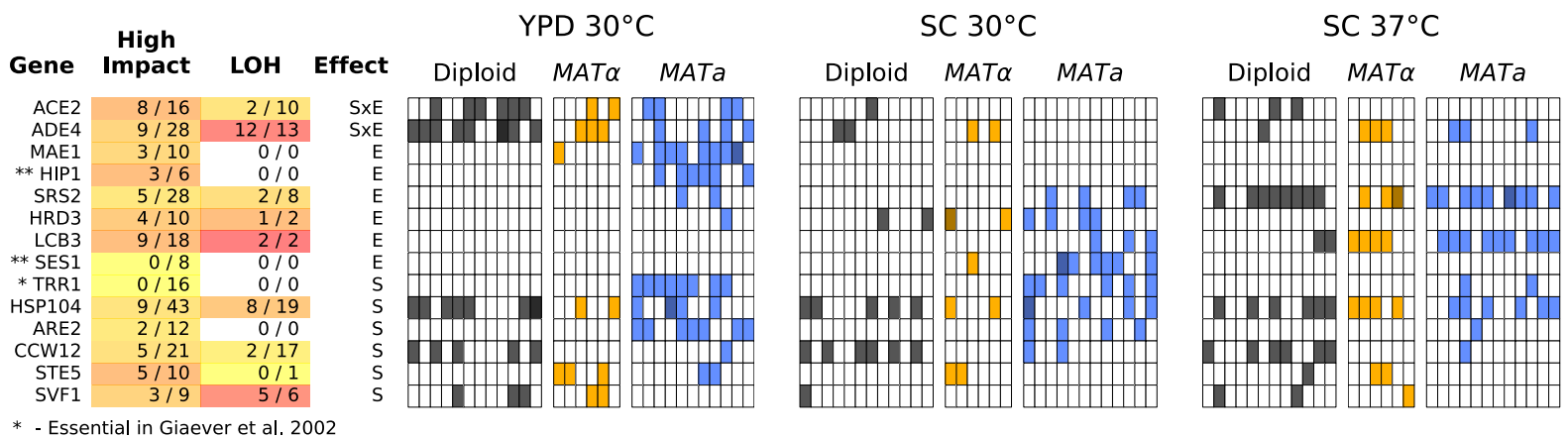

** - Also essential (non-evolvable) in Liu et al. 2015

Figure 6 - figure supplement 5. Same as Figure 6, but for all multi-hit genes where hits are distributed significantly unevenly across strain-types (S), environments (E), or both (SxE) compared to a null model where fixations are not strain or environment dependent. 
A
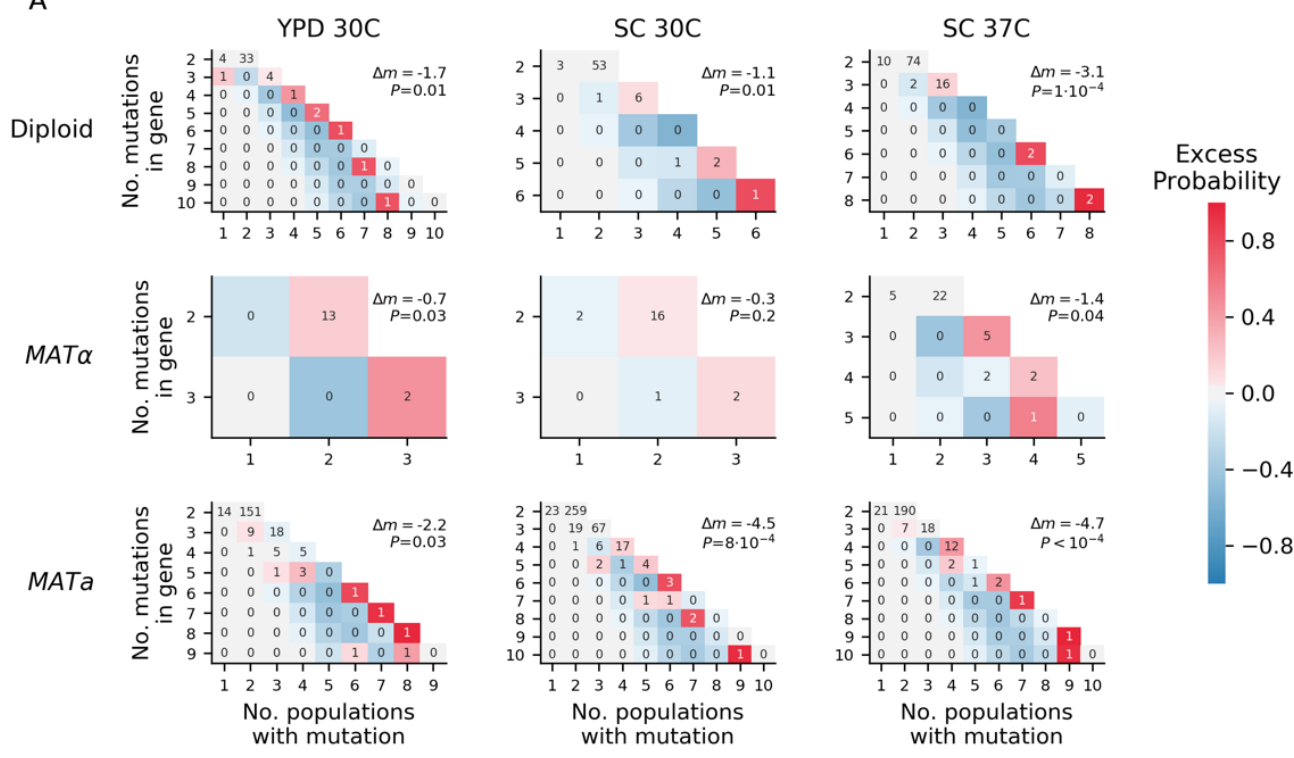

B
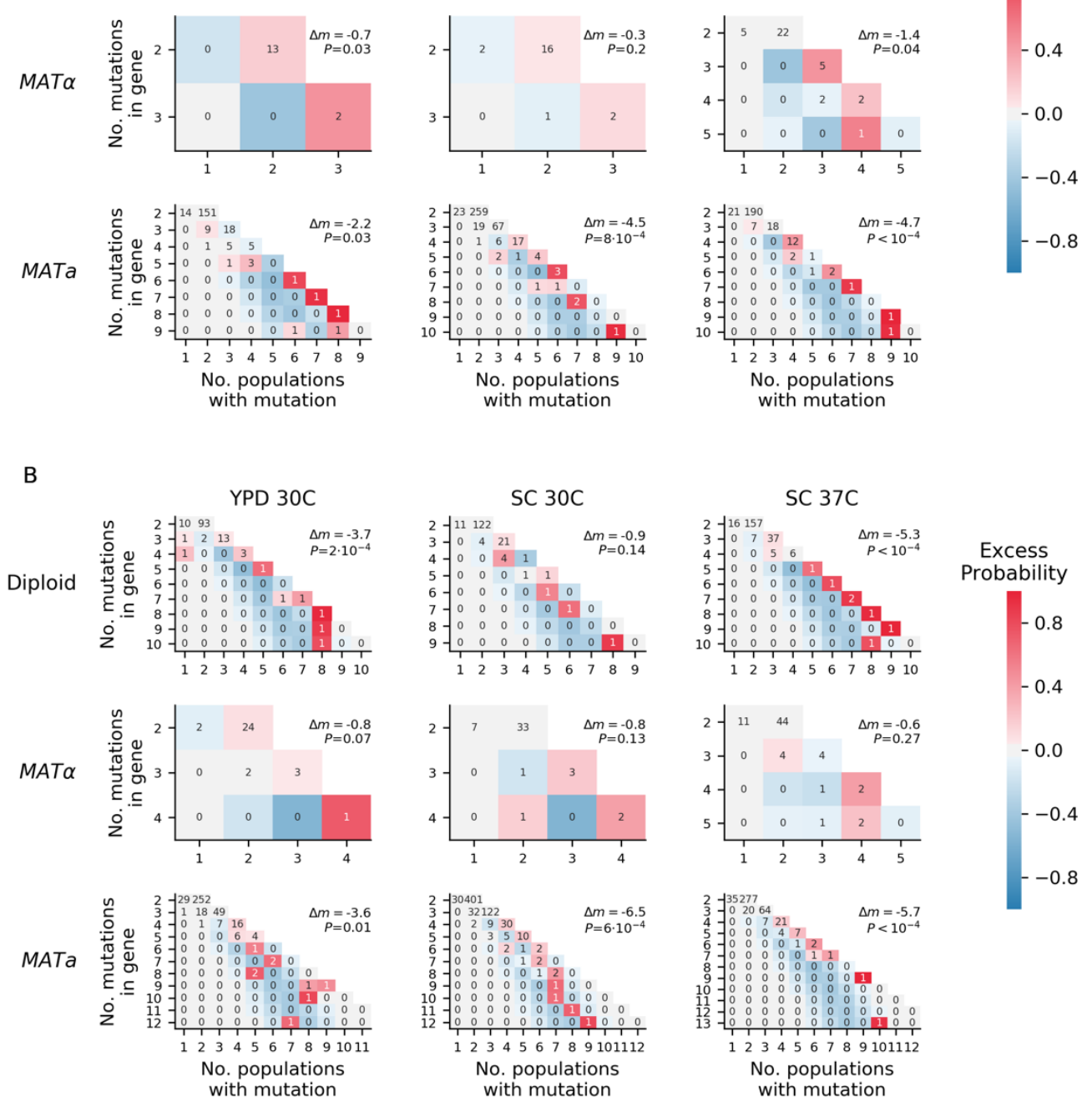

Figure 7 - figure supplement 1. Overdispersion. (A) For each environment-strain combination, we plot the number of genes having a certain number of fixed nonsynonymous mutations (y axis) spread amongst a certain number of unique populations (x axis). Each possible outcome is colored by its excess probability, as compared to a simulated null expectation in which mutations are distributed among populations using a multinomial distribution that takes into account how many nonsynonymous mutations fix in each population. Each plot is annotated with $\Delta m$, the difference between the total number of "missed opportunities" as defined by Good et al. (2017) and the average total number of missed opportunities from simulated datasets, along with the probability of finding less than or equal to our total missed opportunities in one of the simulated datasets. The negative values for $\Delta m$ indicate that we are seeing less missed opportunities than we would expect by chance, indicating overdispersion most likely caused by a "coupon collecting" effect. (B) The same as A, but also including nonsynonymous mutations that are detected but do not fix. 


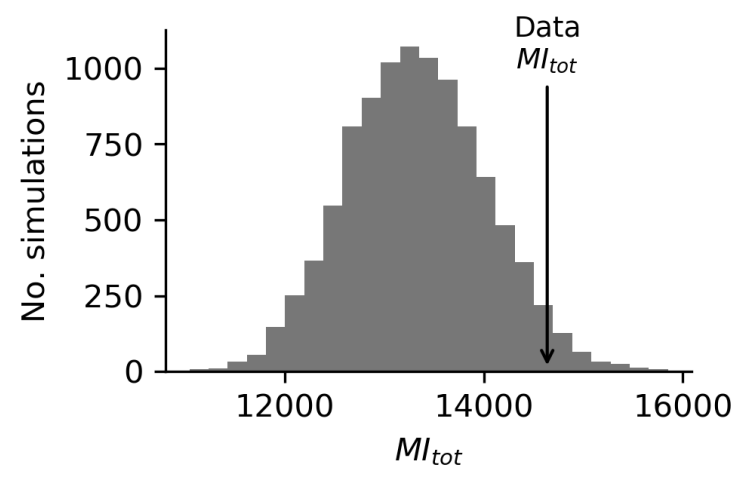

Figure 7 - figure supplement 2. Mutual information analysis. Comparison of the sum of mutual information between all multi-hit genes in our dataset and the mutual information between this set of genes in simulated data based on probabilities assigned to each mutation in each population, allowing for different probabilities in each environment strain combination (see Methods). 


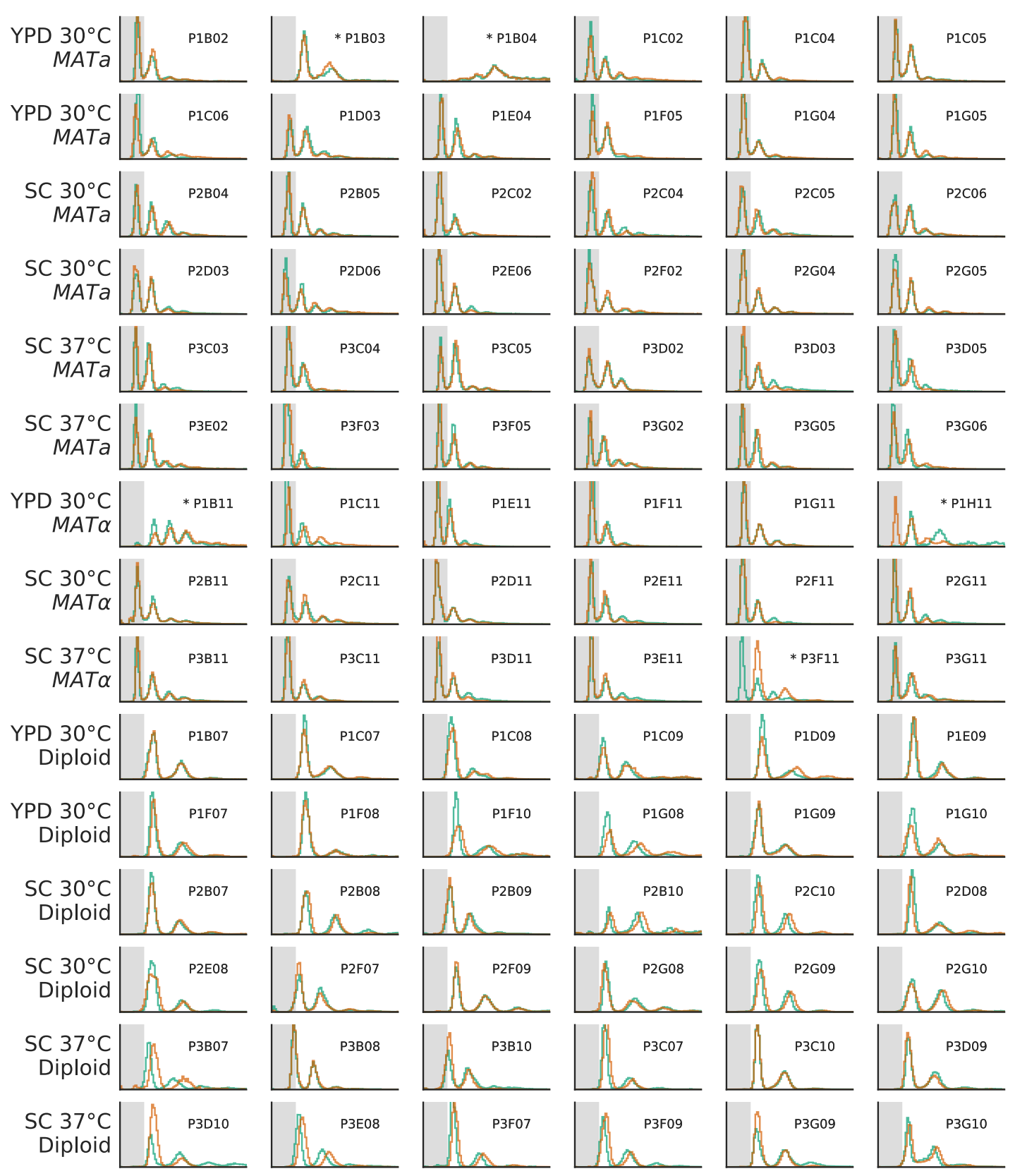

Figure 8 - figure supplement 1. The ploidy state of two clones from each focal population, shown by FITC histograms of Sytox-stained cells. The x-axis is in arbitrary fluorescence units, and the yaxis is normalized frequency. We have shaded the area where single-genome-copy cells (1N) usually fall to help identify haploids. Populations with abnormal FITC histograms are marked by asterisks. P1B03 is the only haploid population that became diploid. Based on sequencing data, this transition likely happened between generation 5000 and generation 7500 (Figure 3 - figure supplement 3). P1H11 and P3F11 both had one diploid and one haploid clone, suggesting that diploids may be present in these populations, but have not fixed. P1B04 and P1B11 have strange FITC histograms, which we believe is due to clustering phenotypes in these populations (Figure 8 figure supplement 2). Based on continued fixations in sequencing data even at the final timepoint, it is unlikely that diploid haplotypes have played a significant role in any of these four populations up to this point in the evolution (Figure 3 - figure supplements 3, 4, and 10). 

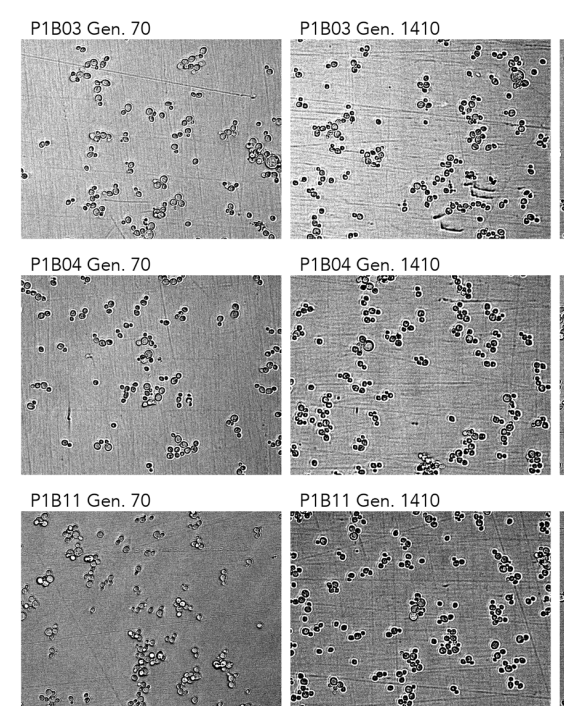

P1B04 Gen. 1410

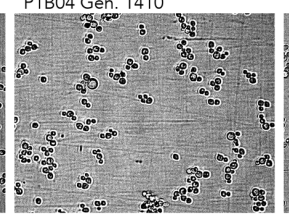

P1B11 Gen. 1410
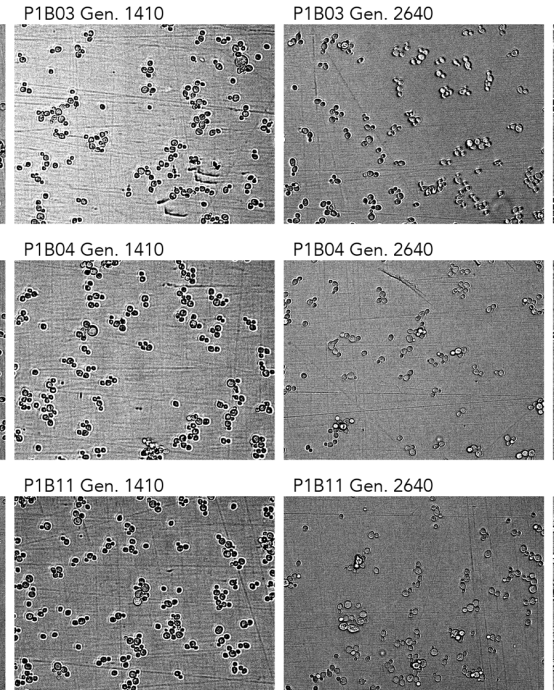

P1B04 Gen. 2640

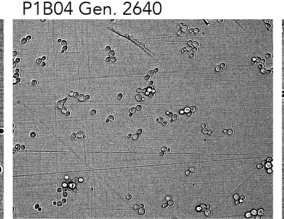

P1B11 Gen. 2640

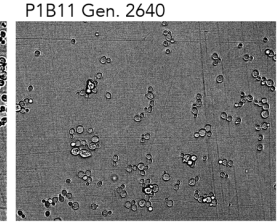

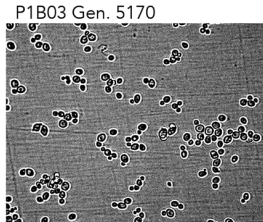

P1B04 Gen. 5170

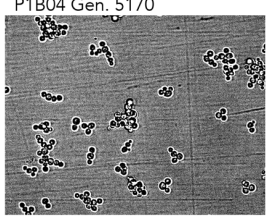

P1B11 Gen. 5170

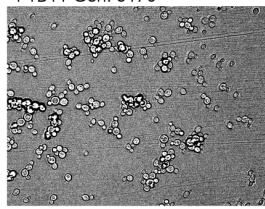

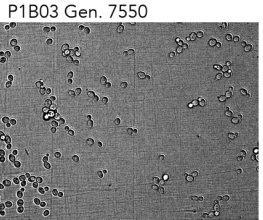

P1B04 Gen. 7550

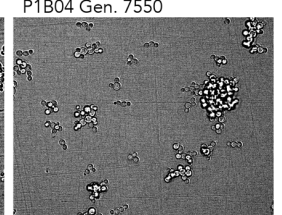

P1B11 Gen. 7550

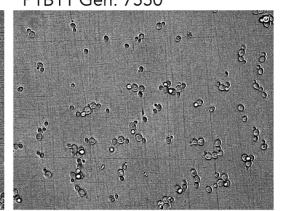

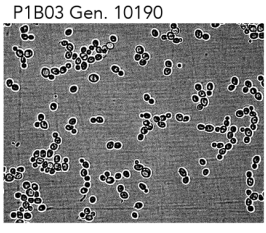

P1B04 Gen. 10190

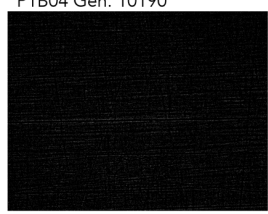

P1B11 Gen. 10190

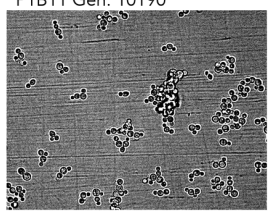

Figure 8 - figure supplement 2. Cell imaging from 3 populations with abnormal Sytox data. Note the clustering phenotypes observed in later timepoints of P1B04 and P1B11. The microscope failed to capture an image for P1B04 generation 10190. All imaging data is available in Supplementary file 6. 
bioRxiv preprint doi: https://doi.org/10.1101/2020.10.09.330191; this version posted October 10, 2020. The copyright holder for this preprint (which was not certified by peer review) is the author/funder, who has granted bioRxiv a license to display the preprint in perpetuity. It is made available under aCC-BY-ND 4.0 International license.
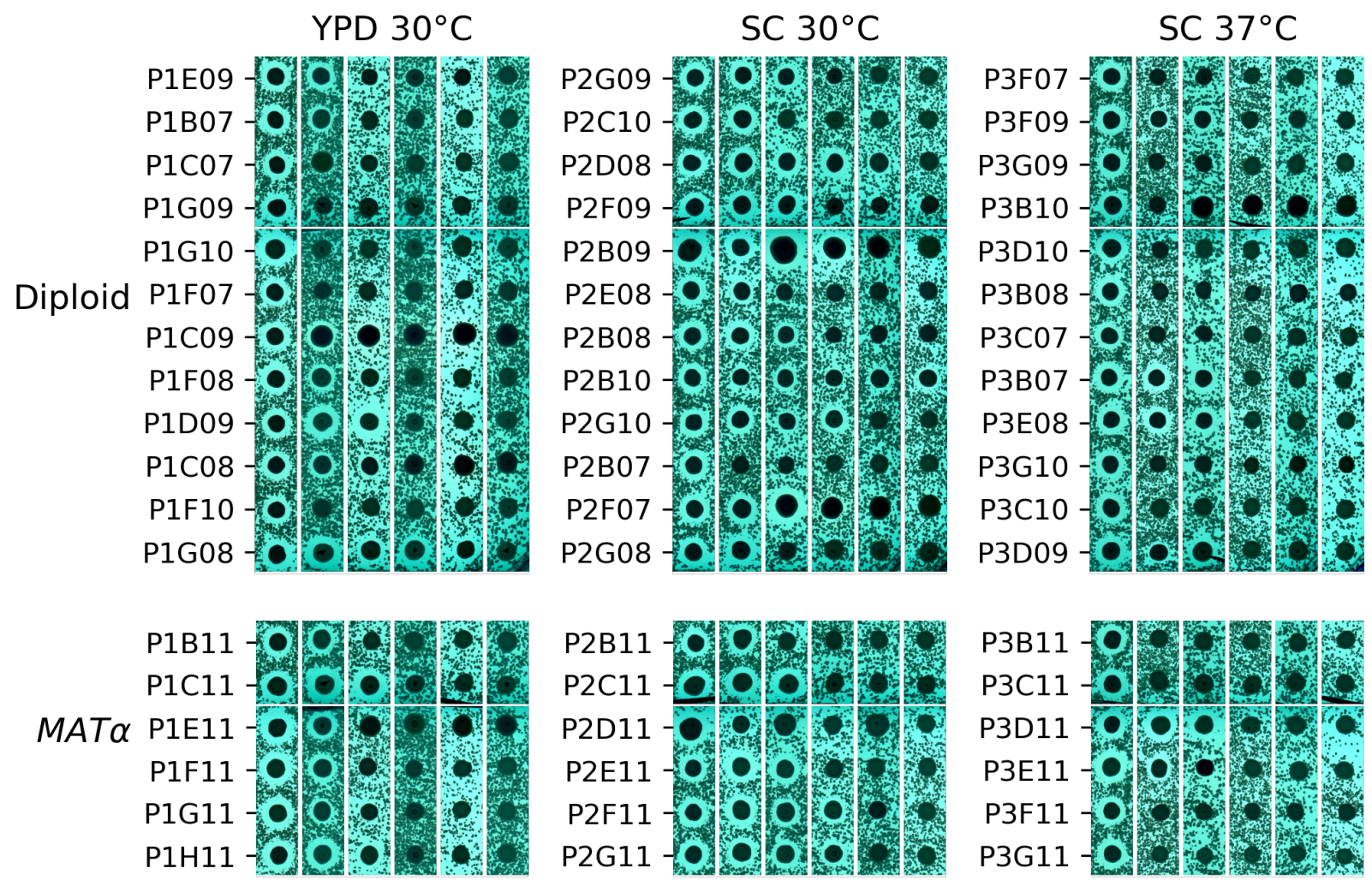

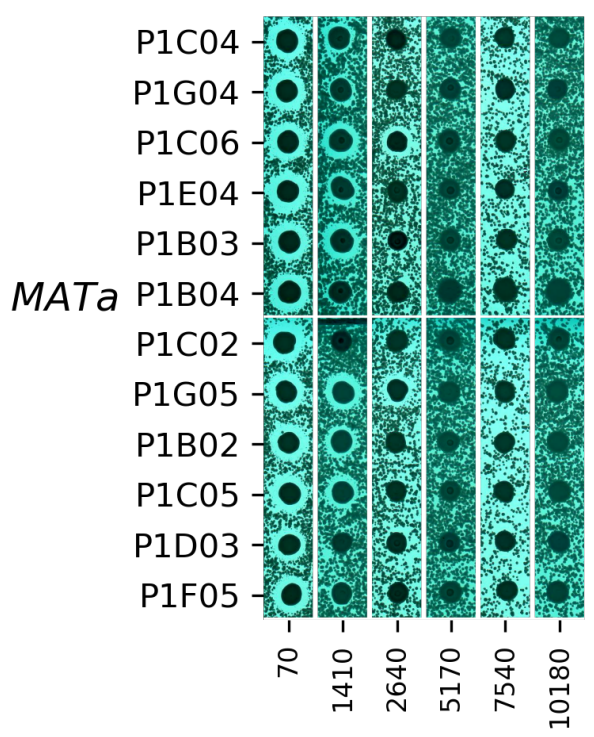

Generation

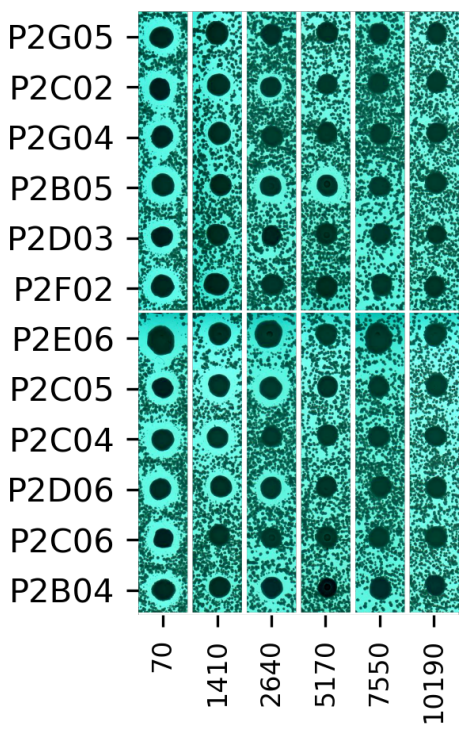

Generation

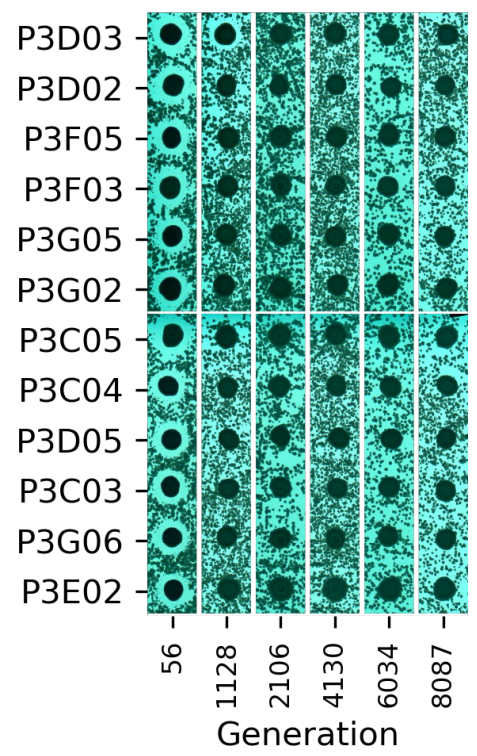

Figure 9 - figure supplement 1. Contrast-enhanced scanned images of killer virus halo assays. 